\title{
The changing chromatome as a driver of disease: A panoramic view from different methodologies
}

\author{
Isabel Espejo1', Luciano Di Croce, ${ }^{1,2,3}$ and Sergi Aranda ${ }^{1}$
}

1. Centre for Genomic Regulation (CRG), Barcelona Institute of Science and Technology, Dr. Aiguader 88, Barcelona 08003, Spain

2. Universitat Pompeu Fabra (UPF), Barcelona, Spain

3. ICREA, Pg. Lluis Companys 23, Barcelona 08010, Spain

${ }^{*}$ Corresponding authors:

Luciano Di Croce (Luciano.DiCroce@crg.eu)

Sergi Aranda (sergi.aranda@crg.eu) 


\section{GRAPHICAL ABSTRACT}

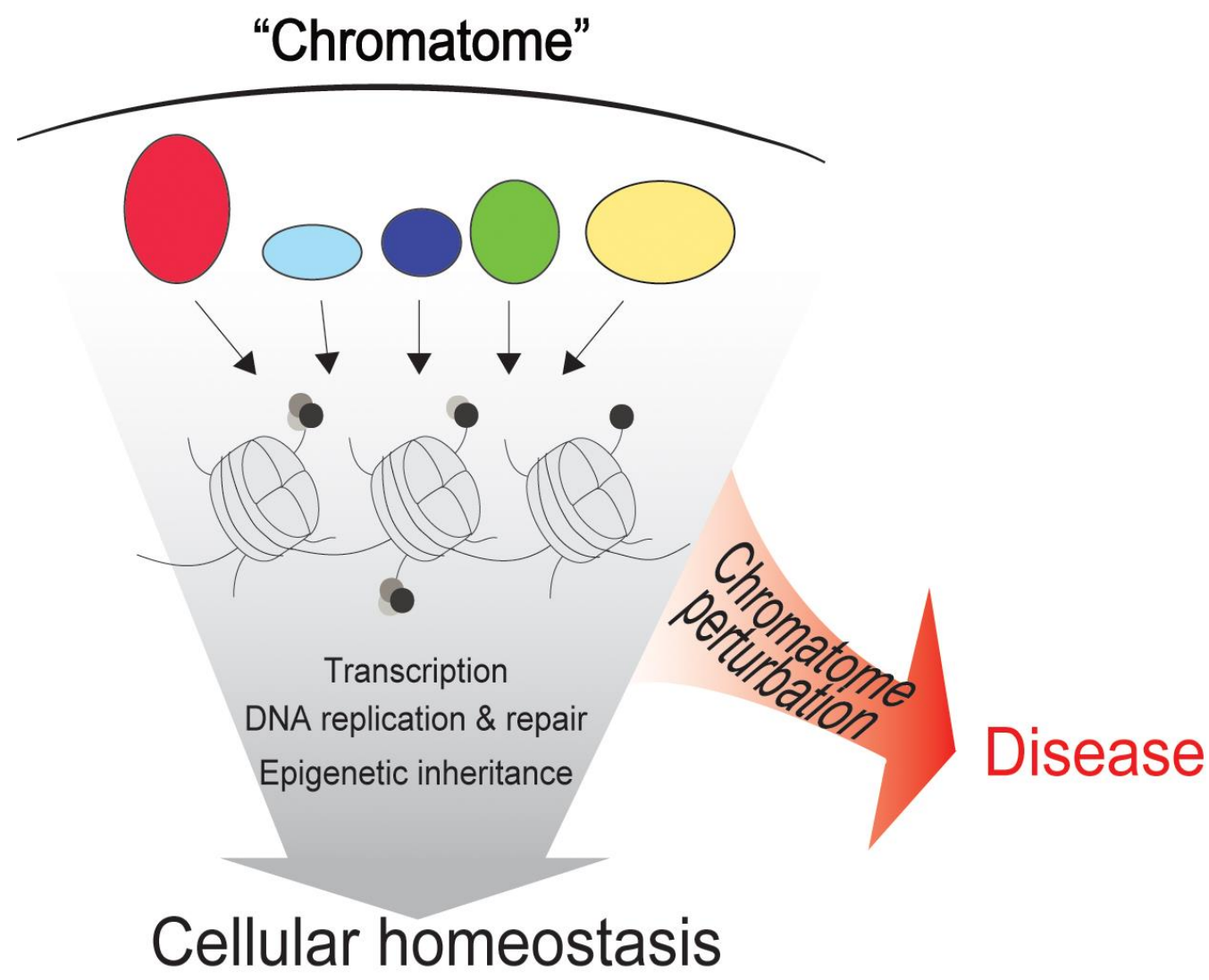

Chromatin-bound proteins regulate gene expression, replicate and repair DNA, and transmit epigenetic information. Several human diseases are highly influenced by alterations in the chromatinbound proteome. Thus, biochemical approaches for the systematic characterization of the chromatome could contribute to identifying new regulators of cellular functionality, including those that are relevant to human disorders. 


\section{SUMMARY}

Chromatin-bound proteins underlie several fundamental cellular functions, such as control of gene expression and the faithful transmission of genetic and epigenetic information. Components of the chromatin proteome (the "chromatome") are essential in human life, and mutations in chromatin-bound proteins are frequently drivers of human diseases, such as cancer. Proteomic characterization of chromatin and de novo identification of chromatin interactors could thus reveal important and perhaps unexpected players implicated in human physiology and disease. Recently, intensive research efforts have focused on developing strategies to characterize the chromatome composition. In this review, we provide an overview of the dynamic composition of the chromatome, highlight the importance of its alterations as a driving force in human disease (and particularly in cancer), and discuss the different approaches to systematically characterize the chromatin-bound proteome in a global manner. 


\section{INTRODUCTION}

Chromatin is the macromolecular complex formed by DNA together with physicallyassociated proteins and non-coding RNAs. At a functional level, the chromatin-bound proteome (the so-called "chromatome") includes numerous different proteins that regulate the tridimensional structure of chromatin fibers within the nucleus as well as correct gene transcription, timely replication of the chromatin, and genomic integrity. $[1,2,3]$ The chromatome comprises very diverse proteins with distinct functional interacting domains, enzymatic activities, isoforms, and/or post-translational modifications (PTMs). ${ }^{[4,5]}$ The spatial distribution of proteins present on chromatin is also very distinct, and some proteins are highly abundant and cover large chromatin regions (e.g. telomeres, centromeres), whereas others are discretely distributed within a few nucleotides. ${ }^{66]}$ Furthermore, the temporal residency of chromatin proteins can vary remarkably, ranging from a few milliseconds for transcription factors (TF) to several hours for stable histone proteins. ${ }^{[7,8]}$ Perturbations in chromatome composition, protein distribution across the genome, and chromatin interaction dynamics are directly responsible for several human diseases, including cancer. [2,9,10,11]

The levels of messenger RNAs (mRNAs) are poor predictors of protein levels; $[12,13]$ [14,15] and do not reflect functional changes affecting protein-chromatin interactions. Thus, experimental chromatome profiling is likely to outperform transcriptome determination on predicting chromatin functionality and organization. ${ }^{[16,17]}$

\section{THE CHROMATIN PROTEOME IS HIGHLY DYNAMIC}

The core nucleosome histones are the most abundant eukaryotic nuclear proteins, with $~ 8,500-17,000$ copies per Mb of genome. ${ }^{[6]}$ Nucleosomes are the functional unit of the chromatin and comprise two of each of the core histones $\mathrm{H} 2 \mathrm{~A}, \mathrm{H} 2 \mathrm{~B}, \mathrm{H} 3$, and H4, which wrap up $\sim 147$ bp of DNA. ${ }^{[18,19]}$ Histones are basic (rich in lysine and arginine) and neutralize the negative charge of DNA. Finally, the linker histone $\mathrm{H} 1$ binds the DNA at the entry and exit of the nucleosomes, thereby increasing their stability and topological organization. [20]

The chromatin topology fluctuates across the genome, from the densely arranged constitutive and facultative heterochromatin to the low nucleosome-occupied euchromatin. ${ }^{[21,22]}$ The stable constitutive heterochromatin generally associates with preservation of genome integrity and accurate chromosome segregation. In contrast, the dynamic interchange between facultative heterochromatin and euchromatin appears essential for regulating the access of the DNA replication, repair, and 
transcription machineries to genomic regulatory regions. [23,24,25] These genomic regulatory regions mainly comprise promoters, enhancers, and insulators. In humans,

The human ATP-dependent CRCs comprises around 100 different proteins classified into four major complex subfamilies: switch/sucrose non-fermentable (SWI/SNF), chromodomain helicase DNA-binding (CHD), imitation switch (ISWI), and inositolrequiring 80 (INO80). ${ }^{[33,34]}$ The four complexes break the histone-DNA interactions using ATP, thereby regulating nucleosome distribution and density across the genome, as well as their predisposition to chemical modifications. ${ }^{[3,35,36,37]}$ Chemical PTMs in nucleosomal histones provide additional regulatory information for the recruitment of other chromatin-bound proteins. ${ }^{[5,8,38,39,40]}$ In humans, over 100 histone 'writer' enzymes, and 50 'eraser' enzymes, have been identified ${ }^{[41]}$ as responsible to introducing or releasing more than 400 different histone PTMs. ${ }^{[42]}$ Methylation and acetylation of lysines at the $\mathrm{N}$-terminal tails of $\mathrm{H} 3$ and $\mathrm{H} 4$ are the most abundant and functionally described PTMs, and phosphorylation and ubiquitination are also wellcharacterized. ${ }^{[43,44,45]}$ The different histone PTMs can recruit histone readers that contain specific histone PTM recognition domains, thus facilitating changes in chromatin topology and genome functionality. ${ }^{[46]}$ For instance, histone lysine acetylation disrupts the histone-DNA electrostatic interactions and mediates the recruitment of one or several of the YEAST- $(n=4)$ and bromodomain- $(n=46)$ containing proteins ${ }^{[39]}$ on active promoters and enhancers. ${ }^{[47]}$ In contrast, histone lysine methylation does not alter the overall charge of chromatin and has a heterogeneous functionality, through the recruitment of histone readers that contain 
Tudor $(n=41)$, PWWP $(n=24)$, PHD $(n=99)$, WDR $(n=361)$, or chromodomains $(n=29)$. ${ }^{[5]}$

DNA chemical modifications can also influence protein interactions with chromatin. In particular, cytosine methylation in $\mathrm{CpG}$ dinucleotides is recognized by methyl-binding proteins with methyl-CpG-binding domains (MBD) $(n=11), \mathrm{C} 2 \mathrm{H} 2$ zinc finger domains $(n=8)$, or SET- and RING finger-associated domains (SRA) $(n=2),{ }^{[48]}$ to mediate transcription, DNA repair, and genome stability. ${ }^{[48,49,50]}$

Finally, different structural proteins contribute to the spatial rearrangements of the chromatin. The CCCTC-binding factor (CTCF) and the protein complex cohesin contribute to the formation of genomic loops. At the megabase scale, these genomic interacting loops separate self-interacting domains, the so-called topologically associated domains (TADs), which can group together several functional units formed by several kilobases of DNA, known as "insulated neighborhoods". [51,52] Overall, chromatin display a notable heterogenic and dynamic proteome composition that regulates all DNA-based processes.

\section{CHROMATOME ALTERATIONS IN HUMAN DISEASE}

Several pieces of evidence indicate that misregulation of chromatome integrity can profoundly influence human diseases (Figure 2).

Within protein coding regions, analysis of exome sequencing data from 60,706 normal individuals identified loss-of-function (LoF) intolerant genes $(n=3230)$ with a lower mutation rate than expected. ${ }^{[53]}$ Notably, almost $1 / 3(n=903)$ of the LoF intolerant genes encode for chromatin-bound proteins (Supplementary Table 1), which suggests that their LoF might confer reproductive or survival disadvantages to carriers. Accordingly, inherited mutations in several of these proteins are causative of multiple developmental deficiencies and intellectual disabilities, including mutations in any of the six SWI/SNF subunit genes (SMARCB1, SMARCA4, SMARCA2, SMARCE1, $A R I D 1 A$, and $A R I D 1 B$ ), which compromise the transcriptional regulation of several neurodevelopmental genes and correlate with high penetrance of Coffin-Siris syndrome. ${ }^{[54]}$

Changes in the DNA sequences responsible for protein recruitment can also disrupt the function of chromatin-bound proteins. Nearly $90 \%$ of all phenotype-associated mutations identified by genome-wide association studies (GWAS) are located in noncoding DNA sequences. ${ }^{[55,56,57]}$ Generally, these mutations associate with open chromatin regions, suggesting that they are located within regulatory DNA elements, such as promoters, enhancers, silencers, splicing branchpoints, and TAD borders. 
[26,58,59,60] For instance, GWAS identified a strong risk variant associated with sporadic cases of Parkinson's disease (PD). This genomic variant localizes within a transcriptional enhancer that regulates the expression of the a-synuclein (SNCA), a key gene associated with PD. At a functional level, the high-risk single-nucleotide polymorphism (SNP) variant disrupts the sequence-specific binding of EMX2 and NKX6-1 transcriptional repressors, thus increasing SNCA expression in neurons to pathological levels. ${ }^{[61]}$

The spontaneous acquisition of somatic mutations in chromatin-bound proteins is also associated to human pathogenesis, and in particular, to cancer. [9,28,29,62] Intensive exome sequencing of thousands of cancer genomes across of multiple cancer types, together with the development of computational algorithms, have enabled cancer driver mutations in specific genes to be identified. ${ }^{[63,64,65,66]}$ Notably, about $50 \%$ of the commonly identified cancer driver genes $\left(n=120\right.$, from $\left.{ }^{[63,64,65,66]}\right)$ encode chromatinbound proteins ( $n=62$ ), strongly underscoring the impact of chromatin deregulation as a primary event establishing cancer malignancy. For instance, TP53 is the most commonly mutated gene across the Pan-Cancer collection ( $>3000$ tumors from 12 major cancer types) and is mutated in about $42 \%$ of the samples. [67] Histone writer and eraser enzymes are among the most mutated category of genes, comprising 13 enzymes ranging from $0.8 \%(E Z H 2)$ to $6.6 \%$ (MLL3) of mutational frequency over the 12 Pan-Cancer types. ${ }^{[67]}$ Strikingly, mutations affecting the methyl transferase of the Polycomb repressive complex 2 (PRC2), EZH2 (which methylates lysine 27 of histone $\mathrm{H} 3$, giving $\mathrm{H} 3 \mathrm{~K} 27 \mathrm{me} / 3)$, ${ }^{[18]}$ is often one of the few genetic abnormalities in cancer genomes with a low mutation burden. Thus, LoF mutations at EZH2 are present in almost $2 \%$ of patients with acute myeloid leukemia $(A M L)$, which harbors the lowest mutation frequency of adult cancers (0.28 mutation per megabase). [67,68]

The recent identification of mutations in histone genes in pediatric cancers, which also usually have a low mutation burden, has reinforced the hypothesis that misregulation of chromatin is a central mechanism in cancer development. In particular, the mutation of the lysine residue 27 of histone $\mathrm{H} 3$ to methionine (H3K27M) is found in up to $80 \%$ of children with diffuse intrinsic pontine gliomas (DIPG), an aggressive pediatric highgrade gliomas. ${ }^{[69,70]}$ Even though H3K27M represents less than $20 \%$ of histone $\mathrm{H} 3$ in DIPG cells, it induces a large misregulation of chromatin by directly compromising the activity of EZH2. Accordingly, H3K27M induces: a widespread decrease of H3K27me3 and H3K27me2, which are essential histones PTMs for chromatin compaction and transcriptional silencing; an increase in H3K27ac, H3K4me3, and H3K36me2; and reduced DNA levels of 5-methylcytosine $(5 \mathrm{mC}) .{ }^{[71,72,73,74,75]}$ Genome-wide occupancy 
studies by ChIP-seq and biochemical analyses indicate the existence of heterotypic H3K27M-K27ac in DIPG cells that colocalizes with bromodomain proteins on actively transcribed genes, where PRC2 is excluded. ${ }^{[76]}$ Remarkably, analysis of the chromatin reorganization of DIPGs has provided the foundation for two promising therapeutic strategies against DIPG, of using either bromodomain and the extraterminal domain (BET) protein or EZH2 inhibitors. [76,77] These suggest that misregulation of histone modifiers, and in general, of chromatin-bound proteins, results in the acquisition of aberrant chromatin organization that can be exploited as vulnerabilities to target tumoral cells. Thus, the identification of changes in the chromatin composition can open new therapeutic approaches in several human diseases.

\section{TECHNICAL CHALLENGES OF CHROMATOME CHARACTERIZATION}

The proteomic characterization of the chromatin (chromatome) is a challenging endeavor due to $(i)$ the difficulty of cleanly separating chromatin material from other cellular organelles, which can result in the presence of contaminants, (ii) the physicochemical heterogeneity of different chromatin regions, which require a balanced stringency of purification, and (iii) the mechanic sensitivity of heterochromatin to external forces, which can perturb the physiological chromatin composition during purification.

During interphase (the period between two cell divisions), chromatin is a water-insoluble polymer that tightly interacts with the nuclear periphery. ${ }^{[78]}$ This property complicates its separation from the highly abundant nuclear lamina, a 10-30 nm thick meshwork of intermediate filaments. ${ }^{[79]}$ Further, chromatin is not uniformly distributed throughout the nucleus but rather displays different densities and physical resistances. For instance, moving from the periphery to the center of the nucleus of human epithelial cells, the chromatin concentration comprises $50 \%$ and $20 \%$ of the local subnuclear volume, respectively. ${ }^{[78]}$ Moreover, in contrast to euchromatin, the dense peripheral constitutive heterochromatin offers resistance to mechanical, enzymatic, and ion-based extraction. [80,81,82] Hence, while chromatin extractions in mild conditions might lead to an incomplete representation of the nuclear heterochromatin, labile euchromatin fragments or weakly bound proteins can be lost during isolation in harsh conditions.

Also, intense chromatin extraction methods from living cells can potentially introduce unpredicted artifacts in their proteomic composition. For instance, at constitutive heterochromatin, histone PTMs are labile upon mild physical perturbations in human epithelial cells. [83] Using low-amplitude stretches in these cells, for periods as short as 30 min, causes a rapid and persistent chromatin loss of about $30 \%$ of H3K9me3 and a notable reduction in the elongating form of RNA polymerase II. [83] Thus, any experimental 
approach aiming to capture the chromatin-bound proteome in its native conditions must consider the rapid mechano-adaptation of the epigenome to external microenvironmentderived forces.

To date, several pioneering studies have developed very elegant biochemical strategies that, in combination with mass spectrometry, aim to investigate the chromatome. According to the broadness of the characterization, these methodologies can be classified as either target-specific or bulk chromatin proteomics. Target-specific methods aim to characterize: i) the proteomic composition of specific-loci, by isolating individual chromatin segments, ${ }^{[84]}$ or ii) the protein-protein interactome within the chromatin, by capturing proteins of interest or specific histone PTMs. [2,85,86,87,88,89] On the other hand, the bulk chromatin proteomic methods aim to provide a global description of nuclear chromatin or large chromatin subtypes. These strategies have provided insight into the mechanisms governing chromatin regulation and organization, characterized the chromatome composition in different biological contexts, and identified de novo chromatin-bound proteins. Below, we discuss the biochemical principles and challenges of bulk chromatin proteomic methods and focus on providing a panoramic view.

\section{CHROMATIN ISOLATION FROM NATIVE NUCLEOUS}

Since the initial protocols described fifty years ago, ${ }^{[90]}$ multiple methods for bulk chromatin isolation from living cells have been developed. These methods rely on limited biochemical principles to obtain a final soluble chromatin material outside of the nucleus for protein analysis (Figure 3). Physically, chromatin is a water-insoluble, stiff, and large polymer confined in the nucleus of eukaryotic cells. [79,90,91] Outside of the nucleus, chromatin self-associates in a condensed thick mass in low ionic strength buffer $(<1 \mathrm{mM})$. [92] This property is used to collect large chromatin fragments upon nuclear breakdown followed by centrifugation. After chromatin precipitation, proteins can be solubilized with ionic detergents, ${ }^{[93,94,95]}$ or by fragmenting DNA into small soluble fragments. [96] Alternatively, small chromatin fragments can be released directly from the nucleus and solubilized by digesting DNA with endonucleases in isotonic conditions (150 mM salt), [97] or by sequential digestion and subsequent salt-extraction. ${ }^{[98]}$ Finally, the electrostatic interactions of proteins with chromatin can be disturbed by increasing the ionic strength of nuclear solutions, thus solubilizing the proteins outside of the nucleus (Figure 3). [82] 


\section{Chromatin isolation by nuclear breakdown}

In 2000, Mendez $\mathrm{J}$ and Stillman B. elaborated a fast and simple low ionic-based extraction method to purify insoluble material highly enriched in tightly bound chromatin proteins. ${ }^{[93]}$ Very similar to a previous methodology, ${ }^{\left[{ }^{11]}\right.}$ Mendez \& Stillman's method is based on a hypotonic swelling step, whereby the cells are lysed in the presence of a nonionic detergent, and the nuclei are collected by low centrifugation. A gentle nuclear breakdown is achieved in a low ionic strength buffer $(<1 \mathrm{mM})$ containing chelating agents (EDTA and EGTA) to further reduce residual divalent cations, and dithiothreitol to reduce disulfide bridges. Chromatin is subsequently collected by centrifugation for posterior analysis. Since its description, this chromatin enrichment strategy has become very popular and has been used both alone to analyze chromatin associations of particular proteins ${ }^{[99]}$ as well as coupled with mass spectrometry-based proteomic studies. [94,95,100,101] Posteriorly, to increase the purity of chromatin preparation, variations in buffer composition and the addition of detergent were introduced to the original protocol to remove nuclear membrane components, as well as non-chromatin nucleoplasmic material. [102,103,104,105,106] Proteomic material from insoluble chromatin pellets was solubilized using ionic detergent-containing buffers (such as sodium dodecyl sulfate, SDS). ${ }^{[93,94,95]}$

\section{Chromatin solubilization by nuclease digestion}

Alternatively, chromatin material can also be solubilized by enzymatic methods that fragment the large insoluble chromatin into small soluble fragments using nonspecific nucleases. Micrococcal nuclease (MNase) can create double-strand breaks within nucleosome linker regions, ${ }^{[107]}$ while DNase I endonuclease preferentially cleaves GCrich DNA unprotected by bound proteins, which are often located in active regulatory regions. [107] Importantly, MNase and DNase I display different digestion kinetics, according to the amount of enzyme used and the time of incubation; [107,108,109] these differences have been exploited to characterize the protein composition in different topological regions of the chromatin ${ }^{[96]}$ as well as proteins with different interaction strengthens with chromatin. ${ }^{[97]}$ For instance, Dutta and collaborators treated insoluble chromatin pellets with endonucleases at different time points and characterized the temporal release of proteins associated with different chromatin types. [96] Proteins released early during treatment with both $\mathrm{MNase}$ and DNase I were enriched in factors associated with active GC-rich promoters or transcription start sites and loosely compacted euchromatin. On the other side, approximately half of non-histone proteins identified in this study belonged to the chromatin pellet, indicative of the large diversity of 
protein composition in resistant heterochromatin. [96] A variation of this enzymatic approach was later adapted by the laboratory of E. Meshorer. ${ }^{[97]}$ The technology (named differential chromatin-associated proteins, D-CAP) uses brief digestion with increasing concentrations of MNase in the presence of salt $(150 \mathrm{mM})$; this leads to the immediate release of small fragments upon digestion. The dose-dependent enzymatic treatment in isolated nuclei simplified the experimental procedure and enabled the authors to standardize the same protocol in different biological conditions. By combining D-CAP with mass spectrometry, Alajem and collaborators explored the differential association of chromatin-bound proteins in pluripotent and differentiated cells. ${ }^{[97]}$ They found that the SWI/SNF-type complex subunits SMARCC1 and SMARCD1 are more tightly associated with chromatin in differentiated cells with respect to pluripotent cells. As both proteins are expressed at similar levels in both cell types, these results point towards a posttranslational regulation of their chromatin association.

\section{Chromatome solubilization by salt extraction}

Protein-nucleic acid interactions are mostly mediated by electrostatic interactions. This property has long been used to differentiate loosely bound from tightly bound chromatin proteins. ${ }^{[110,111,112]}$ Based on the differential physical properties of the chromatin proteins, Avgousti and colleagues recently elaborated a protocol to determine the changes in the chromatin composition of host cells in the presence of the adenoviral protein VII, which resembles cellular histones. ${ }^{[98,113]}$ The protocol relies on the complete MNase digestion of nuclei, in this case in hypotonic conditions, thus preventing the release of chromatin fragments. Subsequent serial washes of the digested nuclei with increasing concentrations of salt $(80-600 \mathrm{mM} \mathrm{NaCl})$ in the presence of nonionic detergent $(0.1 \%$ Triton X-100) enable the sequential release the chromatin material. In these conditions, the proteins weakly bound to chromatin are expected to be released in low-salt concentrations, while tightly chromatin-bound proteins would remain in the insoluble fraction. Using this protocol, the authors found that the viral protein VII causes changes in the chromatome composition of the host cell, with a remarkable increase in chromatin retention of high mobility group protein B family, a key protein involved in the activation of the immune response. ${ }^{[98]}$

To increase proteomic sensitivity, chromatin enriching salt preparations have recently been coupled to data-independent acquisition (CHESS-DIA) mass spectrometry. [82] For this, isolated nuclei are directly subjected to a series of salt-based extraction of nuclear proteins. According to the ionic strength applied, proteins are classified as solubilized in 
four different fractions: unbound nucleoplasmic proteins (15 $\mathrm{mM} \mathrm{NaCl}$ ), euchromatin proteins (250 mM), heterochromatin proteins (600 mM), and insoluble structural proteins. [82] CHESS-DIA offers a notable correlation between proteins classified as chromatin proteins using this protocol and their previous annotation as nuclear proteins in public imaging database (>85\%). However, $55 \%$ of protein classified as nucleoplasmic were not observed in the nucleus by direct immunostaining, ${ }^{[82]}$ thus alerting for the presence of contaminants in the nuclear fraction of CHESS, most likely derived from co-isolated organelles.

In our view, some potential caveats must be taken into consideration when using one of these (now less technically challenging) methods to characterize the chromatin composition. First, insolubility in low ionic conditions is not an exclusive property of chromatin; hence, these isolation methods can eventually carry over impurities from other organelles (such as mitochondria and vesicles) that can co-precipitate with nuclei or chromatin preparations. ${ }^{[14]}$ Second, the presence of ionic conditions and detergents can decrease retention of proteins to chromatin. [91,113,115,116] Thus, highly mobile nuclear proteins can potentially be lost during purification of chromatin. Third, as transient chromatin interactions are a common feature of chromatin-bound proteins, ${ }^{[117]}$ proteins with a minor fraction bound to chromatin could escape the capture during native chromatin isolation. Fourth, the association of mechanical-sensitive chromatin-bound proteins and histone PTMs can be perturbed during extensive sample manipulation. [83] These perturbations are unpredictable and can be overlooked during sample preparation, thus compromising the interpretation of the results.

\section{CHROMATIN ISOLATION FROM CROSSLINKED NUCLEOUS}

Developed in the laboratory of Juri Rappsilber, the chromatin enrichment for proteomics (ChEP) protocol applies extremely stringent washing conditions to remove nonchromatin-bound proteins. [114,118] In this protocol, chromatin-bound proteins are covalently attached by in vivo crosslinking with formaldehyde either directly or indirectly to DNA. This chemical fixation method offers several benefits: it is cell-permeable and rapidly diffuses throughout the cell, thereby crosslinking DNA-protein and protein-protein complexes associated within a distance of less than $2 \AA$. Artefactual crosslinking is very unlikely, as experimentally proven by incubating DNA with high concentrations of BSA (50 $\mathrm{mg} / \mathrm{ml})$ in $1 \%$ of formaldehyde for several days. [119] Using ChEP, non-crosslinked contaminants are then washed away (using high denaturing extraction buffers containing $4 \%$ SDS and $8 \mathrm{M}$ urea, and intermediate RNase digestion reduces the purification of 
nascent RNA-associated proteins. Chromatin is then solubilized by sonication, and the soluble samples are reverse-crosslinked by heating before proteomic analysis. [114,118] Coupled to mass spectrometry, ChEP displays a notable enrichment in proteins associated with DNA- and chromatin-based processes, such as DNA replication, transcription, and repair. But in contrast to classical native chromatin enrichment, the presence of proteins involved in pre-rRNA processing and proteins with unrelated chromatin functions is reduced. [114,118] Thus, combining crosslinking with harsh washing conditions increases the capacity to discriminate true chromatin components from impurities.

Recently, Zaret and collaborators developed a method (Gradient-seq) to successfully discriminate between sonication-sensitive euchromatin from sonication-resistant heterochromatin in human fibroblasts. ${ }^{80]}$ By separating partially sonicated, crosslinked chromatin with linear sucrose gradients, the authors discriminate the dense chromatin (which sediments in the middle of the gradient) from sonicated-sensitive chromatin (at the top of the gradient). Genomic characterization of the fractions indicated that the dense fractions correspond to constitutive heterochromatin dominated by the presence of H3K9me3 and lamin B1, marked by CpG methylation, and enriched in multiple repetitive elements. On the other hand, the sonicated-sensitive chromatin encompasses the DNase-sensitive euchromatic domains and H3K27me3-marked facultative heterochromatin. In combination with mass spectrometry-based proteomics, Gradientseq enabled 217 heterochromatin-specific proteins to be identified, nearly half of them being present in $\mathrm{H} 3 \mathrm{~K} 9 \mathrm{me} 3$ domains. However, the presence of soluble cellular proteins on the top gradient fraction hampered the determination of proteomic composition of the euchromatin. Further functional analyses indicated that a notable proportion of heterochromatin proteins function as repressors of gene activation, and point towards heterochromatin stability as a barrier for cell lineage conversion. [80]

Schübeler and collaborators then adapted a strategy to separate crosslinked DNA-protein complexes with buoyant, density-based fractionation in a cesium chloride ( $\mathrm{CsCl}$ ) gradient (DEMAC, for density-based enrichment for mass spectrometry analysis of chromatin). [3] In this protocol, nuclear fractions are first purified after hypotonic lysis, and the isolated nucleus is crosslinked with formaldehyde and then sonicated, giving fragments ranging in size from $0.1-12 \mathrm{~kb}$. These fragments are recovered using a stable isopycnic density gradient of $1.39 \mathrm{~g} / \mathrm{cm}^{3}$, after $48 \mathrm{~h}$ of centrifugation. Using this protocol, the complete chromatin can be recovered without any topological biases. In comparison to the cellular and nuclear proteome, the chromatin samples have reduced levels of ribosome and 
spliceosome proteins, which are RNA-bound proteins abundantly found in the nucleus, as the result of adding RNase A before centrifugation. However, RNA depletion can also result in the loss of chromatin retention of relevant proteins attached to chromatin in an RNA-dependent manner.

At the beginning of the 2000s, Sharpless and collaborators developed the concept and methodology of "click chemistry". [120] Click chemistry encompasses a group of simple and highly efficient chemical reactions that enable a set of molecules ("building blocks") to be assembled to synthesize new compounds. Since its development, click chemistry has been applied to a wide range of research areas, including chemistry, material science, and biological sciences. ${ }^{[121]}$ In 2011, two different laboratories elaborated in parallel a very similar method to capture nascent DNA during replication from cells in a very efficient manner, by covalently linking the emergent DNA to biotin moieties using click chemistry. [122,123] The methods of isolation of proteins on nascent DNA (iPOND) and the DNAmediated chromatin pulldown (Dm-ChP) rely on an efficient incorporation of the thymidine analog 5-ethynyl-2'-deoxyuridine (EdU) during DNA replication, the addition of a biotin moiety to the incorporated EdU using mild conditions, and streptavidin-biotin affinity to capture sheared EdU-labelled chromatin. [122,123] Inspired by these technologies, we recently elaborated a simple, robust, and straightforward experimental strategy that combines a biochemical method to capture DNA with high-resolution mass spectrometry analysis and a subsequent statistical assessment, for identification of bona fide chromatin-bound proteins. ${ }^{[124,125]}$ We based our strategy on i) whole-genome metabolic labeling with the thymidine analog 5-ethynyl-2'-deoxyuridine (EdU) during several rounds of cell division, in a non-cytotoxic manner; ii) a chemical crosslinking step, which also preserves weak but specific interactions; iii) addition of a biotin moiety to the incorporated EdU, using the click reaction; iv) fragmentation of chromatin; v) streptavidin-biotin affinity purification, to capture chromatin; vi) mass spectrometry analysis; and, vii) Significance Analysis of INTeractome (SAINT), to identify bona fide chromatin-bound proteins. The biochemical procedure stands out for its simplicity, robustness, and usability in a wide range of cells and flexibility for combining with techniques (such as conventional affinity purification and ChIP). The complete procedure enables the identification of Proteins on Total DNA (named iPOTD, based on its biochemical and phonetical similarity with iPOND). [125] Using iPOTD, we have recently provided a broad and comprehensive characterization of the chromatome of murine pluripotent embryonic stem cells. Importantly, we proved the power of this novel strategy by discovering and characterizing the unexpected chromatin association of a major one-carbon pathway metabolic enzyme, namely adenosylhomocysteinase (AHCY), which is involved in the methionine cycle. ${ }^{[124,125]}$ 


\section{CONCLUSIONS AND OUTLOOK}

Chromatin-bound proteins organize the three-dimensional structure of chromatin, regulate gene expression, replicate DNA, and transmit epigenetic information to cellular progeny. $[18,19,126,127,128]$ Remarkably, human diseases are highly influenced by alterations in the chromatin-bound protein composition. [129] Considering that only $40 \%$ of the protein level variability can be attributed to differences in mRNA levels in mammalian cells, ${ }^{[12]}$ the application of chromatome profiling methods is key to fully understanding the functionality of cells, as well as to unveiling disease-driven molecular mechanism.

Chromatin has a highly heterogenic composition and comprises diverse structural features, such that no single isolation method can be used to study all the different aspects of nuclear chromatin. Therefore, the method selected for chromatin analysis should depend on the particular problem to be investigated. However, in our view, an optimal approach to characterize the chromatin composition must: i) be as simple as possible, to increase the yield of isolation and to reduce technical variability in and between laboratories; ii) preserve weak and transient molecular interactions; iii) be robust enough to minimize the presence of impurities from other subcellular organelles; and iv) be as direct as possible, to unequivocally identify uncharacterized chromatin-bound factors.

As there is currently no absolute criterion of purity for chromatin, the de novo identification of chromatin binding proteins requires additional experimental validations, including chromatin immunoprecipitation combined with massive parallel sequencing. In particular, recent studies have characterized the unexpected association of metabolic enzymes to chromatin. [124,130,131,132,133] Chromatin recruitment of metabolic enzymes is considered to be an adaptive cell solution for the highly compartmentalized metabolic and subcellular local demands of metabolites. These strategies can also exploit solutions found by cancer cells that, similar to pluripotent cells, have an elevated proliferation rate. [124,133] Considered as cellular vulnerability points, pharmacological targeting of these enzymes compromises cellular fitness, ${ }^{[124,133]}$ thus indicating that the de novo identification of chromatin-bound proteins can open as of yet unexplored therapeutic avenues against human diseases.

Chromatin fractionation strategies can be instrumental to clarify the protein composition of subnuclear compartments. These membrane-less compartments (e.g. nucleolus, 
enhancer clusters, or Polycomb bodies) are thought to self-organize by two distinct mechanisms: polymer-polymer phase-separation, by which chromatin binders function as a bridge among different chromatin segments, or ii) liquid-liquid phase separation, through multivalent interactions among proteins and other molecules that bind the chromatin. ${ }^{[134]}$ The mechanism by which the subnuclear compartments self-assemble is predicted to impact their physical and functional properties, such as the size and spreading of the subnuclear compartment across multiple genomic loci. Thus, the proteomic characterization of the subnuclear compartments could contribute to gain insights into the fundamental principles governing chromatin compartmentalization by phase separation.

\section{ACKNOWLEDGMENTS}

We thank all the members of Di Croce laboratory for helpful discussions, and V. A. Raker for scientific editing. I.E. is supported by La Caixa INPhINIT program. L.D.C is supported by grants from the Spanish of Economy, Industry and Competitiveness (MEIC) (BFU2016-75008-P), "Fundación Vencer El Cancer" (VEC), the European Regional Development Fund (FEDER), Fundació "La Marató de TV3", and from AGAUR. S.A. is funded by Ramón y Cajal Program MICIU-FSE (RYC2018-025002-I) and ISCIII-FEDER (PI19/01814). We acknowledge support of the Spanish Ministry Science and Innovation to the EMBL partnership, the Centro de Excelencia Severo Ochoa, and the CERCA Programme / Generalitat de Catalunya.

The authors declare no conflict of interest. 


\section{REFERENCES}

[1] Imhof A, Bonaldi T. (2005). "Chromatomics" the analysis of the chromatome. Mol Biosyst, 1(2), 1126.

[2] Noberini R, Sigismondo G, Bonaldi T. (2016). The contribution of mass spectrometry-based proteomics to understanding epigenetics. Epigenomics, 8(3), 429-45.

[3] Ginno PA, Burger L, Seebacher J, lesmantavicius V, Schubeler D. (2018). Cell cycle-resolved chromatin proteomics reveals the extent of mitotic preservation of the genomic regulatory landscape. Nat Commun, 9(1), 4048.

[4] Aebersold R, Agar JN, Amster IJ, Baker MS, Bertozzi CR, Boja ES, . . Zhang B. (2018). How many human proteoforms are there? Nat Chem Biol, 14(3), 206-14.

[5] Arrowsmith $\mathrm{CH}$, Schapira M. (2019). Targeting non-bromodomain chromatin readers. Nat Struct Mol Biol, 26(10), 863-9.

[6] Bonnet J, Lindeboom RGH, Pokrovsky D, Stricker G, Celik MH, Rupp RAW, . . Muller J. (2019). Quantification of Proteins and Histone Marks in Drosophila Embryos Reveals Stoichiometric Relationships Impacting Chromatin Regulation. Dev Cell, 51(5), 632-44 e6.

[7] Poorey K, Viswanathan R, Carver MN, Karpova TS, Cirimotich SM, McNally JG, ... Auble DT. (2013). Measuring chromatin interaction dynamics on the second time scale at single-copy genes. Science, 342(6156), 369-72.

[8] Wierer M, Mann M. (2016). Proteomics to study DNA-bound and chromatin-associated gene regulatory complexes. Hum Mol Genet, 25(R2), R106-R14.

[9] Dawson MA, Kouzarides T. (2012). Cancer epigenetics: from mechanism to therapy. Cell, 150(1), 1227.

[10] Eubanks CG, Dayebgadoh G, Liu X, Washburn MP. (2017). Unravelling the biology of chromatin in health and cancer using proteomic approaches. Expert Rev Proteomics, 14(10), 905-15.

[11] Marchione DM, Garcia BA, Wojcik J. (2019). Proteomic approaches for cancer epigenetics research. Expert Rev Proteomics, 16(1), 33-47.

[12] Schwanhausser B, Busse D, Li N, Dittmar G, Schuchhardt J, Wolf J, . . Selbach M. (2011). Global quantification of mammalian gene expression control. Nature, 473(7347), 337-42.

[13] de Sousa Abreu R, Penalva LO, Marcotte EM, Vogel C. (2009). Global signatures of protein and mRNA expression levels. Mol Biosyst, 5(12), 1512-26.

[14] Maier T, Guell M, Serrano L. (2009). Correlation of mRNA and protein in complex biological samples. FEBS Lett, 583(24), 3966-73.

[15] Vogel C, Abreu Rde S, Ko D, Le SY, Shapiro BA, Burns SC, . . Penalva LO. (2010). Sequence signatures and mRNA concentration can explain two-thirds of protein abundance variation in a human cell line. Mol Syst Biol, 6, 400.

[16] Wang J, Ma Z, Carr SA, Mertins P, Zhang H, Zhang Z, . . Zhang B. (2017). Proteome Profiling Outperforms Transcriptome Profiling for Coexpression Based Gene Function Prediction. Mol Cell Proteomics, 16(1), 121-34.

[17] Kustatscher G, Grabowski P, Schrader TA, Passmore JB, Schrader M, Rappsilber J. (2019). Coregulation map of the human proteome enables identification of protein functions. Nat Biotechnol, 37(11), 1361-71. 
[18] Aranda S, Mas G, Di Croce L. (2015). Regulation of gene transcription by Polycomb proteins. Sci Adv, 1(11), e1500737.

[19] Cramer P. (2014). A tale of chromatin and transcription in 100 structures. Cell, 159(5), 985-94.

[20] Fyodorov DV, Zhou BR, Skoultchi Al, Bai Y. (2018). Emerging roles of linker histones in regulating chromatin structure and function. Nat Rev Mol Cell Biol, 19(3), 192-206.

[21] Klemm SL, Shipony Z, Greenleaf WJ. (2019). Chromatin accessibility and the regulatory epigenome. Nat Rev Genet, 20(4), 207-20.

[22] Allshire RC, Madhani HD. (2018). Ten principles of heterochromatin formation and function. Nat Rev Mol Cell Biol, 19(4), 229-44.

[23] Saksouk N, Simboeck E, Dejardin J. (2015). Constitutive heterochromatin formation and transcription in mammals. Epigenetics Chromatin, 8, 3.

[24] Stewart-Morgan KR, Reveron-Gomez N, Groth A. (2019). Transcription Restart Establishes Chromatin Accessibility after DNA Replication. Mol Cell, 75(2), 284-97 e6.

[25] Thurman RE, Rynes E, Humbert R, Vierstra J, Maurano MT, Haugen E, ... Stamatoyannopoulos JA. (2012). The accessible chromatin landscape of the human genome. Nature, 489(7414), 75-82.

[26] Hnisz D, Abraham BJ, Lee TI, Lau A, Saint-Andre V, Sigova AA, . . Young RA. (2013). Superenhancers in the control of cell identity and disease. Cell, 155(4), 934-47.

[27] Lambert SA, Jolma A, Campitelli LF, Das PK, Yin Y, Albu M, ... Weirauch MT. (2018). The Human Transcription Factors. Cell, 172(4), 650-65.

[28] Lee TI, Young RA. (2013). Transcriptional regulation and its misregulation in disease. Cell, 152(6), 1237-51.

[29] Bradner JE, Hnisz D, Young RA. (2017). Transcriptional Addiction in Cancer. Cell, 168(4), 629-43.

[30] Young RA. (2011). Control of the embryonic stem cell state. Cell, 144(6), 940-54.

[31] Iwafuchi-Doi M, Donahue G, Kakumanu A, Watts JA, Mahony S, Pugh BF, .. Zaret KS. (2016). The Pioneer Transcription Factor FoxA Maintains an Accessible Nucleosome Configuration at Enhancers for Tissue-Specific Gene Activation. Mol Cell, 62(1), 79-91.

[32] Brahma S, Henikoff S. (2020). Epigenome Regulation by Dynamic Nucleosome Unwrapping. Trends Biochem Sci, 45(1), 13-26.

[33] Hota SK, Bruneau BG. (2016). ATP-dependent chromatin remodeling during mammalian development. Development, 143(16), 2882-97.

[34] Clapier CR, Iwasa J, Cairns BR, Peterson CL. (2017). Mechanisms of action and regulation of ATPdependent chromatin-remodelling complexes. Nat Rev Mol Cell Biol, 18(7), 407-22.

[35] Narlikar GJ, Sundaramoorthy R, Owen-Hughes T. (2013). Mechanisms and functions of ATPdependent chromatin-remodeling enzymes. Cell, 154(3), 490-503.

[36] Sokpor G, Castro-Hernandez R, Rosenbusch J, Staiger JF, Tuoc T. (2018). ATP-Dependent Chromatin Remodeling During Cortical Neurogenesis. Front Neurosci, 12, 226.

Talbert PB, Henikoff S. (2017). Histone variants on the move: substrates for chromatin dynamics. Nat Rev Mol Cell Biol, 18(2), 115-26. 
[38] Strahl BD, Allis CD. (2000). The language of covalent histone modifications. Nature, 403(6765), 415.

[39] Zaware N, Zhou MM. (2019). Bromodomain biology and drug discovery. Nat Struct Mol Biol, 26(10), 870-9.

[40] Janssen KA, Sidoli S, Garcia BA. (2017). Recent Achievements in Characterizing the Histone Code and Approaches to Integrating Epigenomics and Systems Biology. Methods Enzymol, 586, 359-78.

[41] Khare SP, Habib F, Sharma R, Gadewal N, Gupta S, Galande S. (2012). HIstome--a relational knowledgebase of human histone proteins and histone modifying enzymes. Nucleic Acids Res, 40(Database issue), D337-42.

[42] Huang H, Sabari BR, Garcia BA, Allis CD, Zhao Y. (2014). SnapShot: histone modifications. Cell, 159(2), 458- e1.

[43] Zhao Y, Garcia BA. (2015). Comprehensive Catalog of Currently Documented Histone Modifications. Cold Spring Harbor Perspectives in Biology, 7(9).

[44] Sidoli S, Lopes M, Lund PJ, Goldman N, Fasolino M, Coradin M, . . Garcia BA. (2019). A mass spectrometry-based assay using metabolic labeling to rapidly monitor chromatin accessibility of modified histone proteins. Sci Rep, 9(1), 13613.

[45] Simithy J, Sidoli S, Garcia BA. (2018). Integrating Proteomics and Targeted Metabolomics to Understand Global Changes in Histone Modifications. Proteomics, 18(18), e1700309.

[46] Ji X, Dadon DB, Abraham BJ, Lee TI, Jaenisch R, Bradner JE, Young RA. (2015). Chromatin proteomic profiling reveals novel proteins associated with histone-marked genomic regions. Proc Natl Acad Sci U S A, 112(12), 3841-6.

[47] Fujisawa T, Filippakopoulos P. (2017). Functions of bromodomain-containing proteins and their roles in homeostasis and cancer. Nat Rev Mol Cell Biol, 18(4), 246-62.

[48] Mahmood N, Rabbani SA. (2019). DNA Methylation Readers and Cancer: Mechanistic and Therapeutic Applications. Front Oncol, 9, 489.

[49] Jones PA. (2012). Functions of DNA methylation: islands, start sites, gene bodies and beyond. Nat Rev Genet, 13(7), 484-92.

[50] Zhu H, Wang G, Qian J. (2016). Transcription factors as readers and effectors of DNA methylation. Nat Rev Genet, 17(9), 551-65.

[51] Zheng H, Xie W. (2019). The role of 3D genome organization in development and cell differentiation. Nat Rev Mol Cell Biol, 20(9), 535-50.

[52] Hnisz D, Day DS, Young RA. (2016). Insulated Neighborhoods: Structural and Functional Units of Mammalian Gene Control. Cell, 167(5), 1188-200.

[53] Lek M, Karczewski KJ, Minikel EV, Samocha KE, Banks E, Fennell T, . . Exome Aggregation C. (2016). Analysis of protein-coding genetic variation in 60,706 humans. Nature, 536(7616), 285-91.

[54] Tsurusaki Y, Okamoto N, Ohashi H, Kosho T, Imai Y, Hibi-Ko Y, . . Matsumoto N. (2012). Mutations affecting components of the SWI/SNF complex cause Coffin-Siris syndrome. Nat Genet, 44(4), 3768.

[55] Maurano MT, Humbert R, Rynes E, Thurman RE, Haugen E, Wang H, . . Stamatoyannopoulos JA. (2012). Systematic localization of common disease-associated variation in regulatory DNA. Science, 337(6099), 1190-5. 
[56] Fadason T, Schierding W, Lumley T, O'Sullivan JM. (2018). Chromatin interactions and expression quantitative trait loci reveal genetic drivers of multimorbidities. Nat Commun, 9(1), 5198.

[57] Gloss BS, Dinger ME. (2018). Realizing the significance of noncoding functionality in clinical genomics. Exp Mol Med, 50(8), 97.

[58] Chabot B, Shkreta L. (2016). Defective control of pre-messenger RNA splicing in human disease. J Cell Biol, 212(1), 13-27.

[59] Lupianez DG, Kraft K, Heinrich V, Krawitz P, Brancati F, Klopocki E, . . . Mundlos S. (2015). Disruptions of topological chromatin domains cause pathogenic rewiring of gene-enhancer interactions. Cell, 161(5), 1012-25.

[60] Anna A, Monika G. (2018). Splicing mutations in human genetic disorders: examples, detection, and confirmation. J Appl Genet, 59(3), 253-68.

[61] Soldner F, Stelzer Y, Shivalila CS, Abraham BJ, Latourelle JC, Barrasa MI, . . Jaenisch R. (2016). Parkinson-associated risk variant in distal enhancer of alpha-synuclein modulates target gene expression. Nature, 533(7601), 95-9.

[62] Valencia AM, Kadoch C. (2019). Chromatin regulatory mechanisms and therapeutic opportunities in cancer. Nat Cell Biol, 21(2), 152-61.

[63] Bailey MH, Tokheim C, Porta-Pardo E, Sengupta S, Bertrand D, Weerasinghe A, ... Ding L. (2018). Comprehensive Characterization of Cancer Driver Genes and Mutations. Cell, 174(4), 1034-5.

[64] Lawrence MS, Stojanov P, Mermel CH, Robinson JT, Garraway LA, Golub TR, .. Getz G. (2014). Discovery and saturation analysis of cancer genes across 21 tumour types. Nature, 505(7484), 495501.

[65] Martincorena I, Raine KM, Gerstung M, Dawson KJ, Haase K, Van Loo P, . . Campbell PJ. (2017). Universal Patterns of Selection in Cancer and Somatic Tissues. Cell, 171(5), 1029-41 e21.

[66] Dietlein F, Weghorn D, Taylor-Weiner A, Richters A, Reardon B, Liu D, . . Sunyaev SR. (2020). Identification of cancer driver genes based on nucleotide context. Nat Genet, 52(2), 208-18.

[67] Kandoth C, McLellan MD, Vandin F, Ye K, Niu B, Lu C, ... Ding L. (2013). Mutational landscape and significance across 12 major cancer types. Nature, 502(7471), 333-9.

[68] Lund K, Adams PD, Copland M. (2014). EZH2 in normal and malignant hematopoiesis. Leukemia, 28(1), 44-9.

[69] Schwartzentruber J, Korshunov A, Liu XY, Jones DT, Pfaff E, Jacob K, . . Jabado N. (2012). Driver mutations in histone H3.3 and chromatin remodelling genes in paediatric glioblastoma. Nature, 482(7384), 226-31.

[70] Wu G, Broniscer A, McEachron TA, Lu C, Paugh BS, Becksfort J, . . St. Jude Children's Research Hospital-Washington University Pediatric Cancer Genome P. (2012). Somatic histone H3 alterations in pediatric diffuse intrinsic pontine gliomas and non-brainstem glioblastomas. Nat Genet, 44(3), 2513.

[71] Bender S, Tang Y, Lindroth AM, Hovestadt V, Jones DT, Kool M, . . Pfister SM. (2013). Reduced H3K27me3 and DNA hypomethylation are major drivers of gene expression in K27M mutant pediatric high-grade gliomas. Cancer Cell, 24(5), 660-72.

[72] Chan KM, Fang D, Gan H, Hashizume R, Yu C, Schroeder M, . . Zhang Z. (2013). The histone H3.3K27M mutation in pediatric glioma reprograms H3K27 methylation and gene expression. Genes Dev, 27(9), 985-90. 
[73] Lewis PW, Muller MM, Koletsky MS, Cordero F, Lin S, Banaszynski LA, . . Allis CD. (2013). Inhibition of PRC2 activity by a gain-of-function H3 mutation found in pediatric glioblastoma. Science, 340(6134), 857-61.

[74] Venneti S, Garimella MT, Sullivan LM, Martinez D, Huse JT, Heguy A, . . Judkins AR. (2013). Evaluation of histone 3 lysine 27 trimethylation (H3K27me3) and enhancer of Zest 2 (EZH2) in pediatric glial and glioneuronal tumors shows decreased H3K27me3 in H3F3A K27M mutant glioblastomas. Brain Pathol, 23(5), 558-64.

[75] Larson JD, Kasper LH, Paugh BS, Jin H, Wu G, Kwon CH, . . Baker SJ. (2019). Histone H3.3 K27M Accelerates Spontaneous Brainstem Glioma and Drives Restricted Changes in Bivalent Gene Expression. Cancer Cell, 35(1), 140-55 e7.

[76] Piunti A, Hashizume R, Morgan MA, Bartom ET, Horbinski CM, Marshall SA, ... Shilatifard A. (2017). Therapeutic targeting of polycomb and BET bromodomain proteins in diffuse intrinsic pontine gliomas. Nat Med, 23(4), 493-500.

[77] Mohammad F, Weissmann S, Leblanc B, Pandey DP, Hojfeldt JW, Comet I, . . Helin K. (2017). EZH2 is a potential therapeutic target for H3K27M-mutant pediatric gliomas. Nat Med, 23(4), 483-92.

[78] Ou HD, Phan S, Deerinck TJ, Thor A, Ellisman MH, O'Shea CC. (2017). ChromEMT: Visualizing 3D chromatin structure and compaction in interphase and mitotic cells. Science, 357(6349).

[79] Stephens AD, Banigan EJ, Marko JF. (2019). Chromatin's physical properties shape the nucleus and its functions. Curr Opin Cell Biol, 58, 76-84.

[80] Becker JS, McCarthy RL, Sidoli S, Donahue G, Kaeding KE, He Z, . . Zaret KS. (2017). Genomic and Proteomic Resolution of Heterochromatin and Its Restriction of Alternate Fate Genes. Mol Cell, 68(6), 1023-37 e15.

[81] Teves SS, Henikoff S. (2012). Salt fractionation of nucleosomes for genome-wide profiling. Methods Mol Biol, 833, 421-32.

[82] Federation AJ, Nandakumar V, Searle BC, Stergachis A, Wang H, Pino LK, ... Stamatoyannopoulos JA. (2020). Highly Parallel Quantification and Compartment Localization of Transcription Factors and Nuclear Proteins. Cell Rep, 30(8), 2463-71 e5.

[83] Nava MM, Miroshnikova YA, Biggs LC, Whitefield DB, Metge F, Boucas J, . . Wickstrom SA. (2020). Heterochromatin-Driven Nuclear Softening Protects the Genome against Mechanical Stress-Induced Damage. Cell, 181(4), 800-17 e22.

[84] Gauchier M, van Mierlo G, Vermeulen M, Dejardin J. (2020). Purification and enrichment of specific chromatin loci. Nat Methods, 17(4), 380-9.

[85] Ummethum H, Hamperl S. (2020). Proximity Labeling Techniques to Study Chromatin. Front Genet, $11,450$.

[86] Rafiee MR, Girardot C, Sigismondo G, Krijgsveld J. (2016). Expanding the Circuitry of Pluripotency by Selective Isolation of Chromatin-Associated Proteins. Mol Cell, 64(3), 624-35.

[87] Kleiner RE, Hang LE, Molloy KR, Chait BT, Kapoor TM. (2018). A Chemical Proteomics Approach to Reveal Direct Protein-Protein Interactions in Living Cells. Cell Chem Biol, 25(1), 110-20 e3.

[88] Villasenor R, Pfaendler R, Ambrosi C, Butz S, Giuliani S, Bryan E, . . Baubec T. (2020). ChromID identifies the protein interactome at chromatin marks. Nat Biotechnol, 38(6), 728-36.

[89] Burton AJ, Haugbro M, Gates LA, Bagert JD, Allis CD, Muir TW. (2020). In situ chromatin interactomics using a chemical bait and trap approach. Nature Chemistry, 12(6), 520-7. 
[90] Garrard WT, Hancock R. (1978). Preparation of chromatin from animal tissues and cultured cells. Methods Cell Biol, 17, 27-50.

[91] Hancock R, Faber AJ, Fakan S. (1977). Isolation of interphase chromatin structures from cultured cells. Methods Cell Biol, 15, 127-47.

[92] Cai S, Song Y, Chen C, Shi J, Gan L. (2018). Natural chromatin is heterogeneous and self-associates in vitro. Mol Biol Cell, 29(13), 1652-63.

[93] Mendez J, Stillman B. (2000). Chromatin association of human origin recognition complex, cdc6, and minichromosome maintenance proteins during the cell cycle: assembly of prereplication complexes in late mitosis. Mol Cell Biol, 20(22), 8602-12.

[94] Shiio Y, Eisenman RN, Yi EC, Donohoe S, Goodlett DR, Aebersold R. (2003). Quantitative proteomic analysis of chromatin-associated factors. J Am Soc Mass Spectrom, 14(7), 696-703.

[95] Torrente MP, Zee BM, Young NL, Baliban RC, LeRoy G, Floudas CA, . . Garcia BA. (2011). Proteomic interrogation of human chromatin. PLoS One, 6(9), e24747.

[96] Dutta B, Adav SS, Koh CG, Lim SK, Meshorer E, Sze SK. (2012). Elucidating the temporal dynamics of chromatin-associated protein release upon DNA digestion by quantitative proteomic approach. $J$ Proteomics, 75(17), 5493-506.

[97] Alajem A, Biran A, Harikumar A, Sailaja BS, Aaronson Y, Livyatan I, . . Meshorer E. (2015). Differential association of chromatin proteins identifies BAF60a/SMARCD1 as a regulator of embryonic stem cell differentiation. Cell Rep, 10(12), 2019-31.

[98] Avgousti DC, Herrmann C, Kulej K, Pancholi NJ, Sekulic N, Petrescu J, . . Weitzman MD. (2016). A core viral protein binds host nucleosomes to sequester immune danger signals. Nature, 535(7610), 173-7.

[99] Wysocka J, Reilly PT, Herr W. (2001). Loss of HCF-1-chromatin association precedes temperatureinduced growth arrest of tsBN67 cells. Mol Cell Biol, 21(11), 3820-9.

[100] Mulvey CM, Breckels LM, Geladaki A, Britovsek NK, Nightingale DJH, Christoforou A, .. . Lilley KS. (2017). Using hyperLOPIT to perform high-resolution mapping of the spatial proteome. Nat Protoc, 12(6), 1110-35.

[101] Geladaki A, Kocevar Britovsek N, Breckels LM, Smith TS, Vennard OL, Mulvey CM, ... Lilley KS. (2019). Combining LOPIT with differential ultracentrifugation for high-resolution spatial proteomics. Nat Commun, 10(1), 331.

[102] Monte E, Mouillesseaux K, Chen H, Kimball T, Ren S, Wang Y, . . Franklin S. (2013). Systems proteomics of cardiac chromatin identifies nucleolin as a regulator of growth and cellular plasticity in cardiomyocytes. Am J Physiol Heart Circ Physiol, 305(11), H1624-38.

[103] Franklin S, Zhang MJ, Chen H, Paulsson AK, Mitchell-Jordan SA, Li Y, . . Vondriska TM. (2011). Specialized compartments of cardiac nuclei exhibit distinct proteomic anatomy. Mol Cell Proteomics, 10(1), M110 000703.

[104] Franklin S, Chen H, Mitchell-Jordan S, Ren S, Wang Y, Vondriska TM. (2012). Quantitative analysis of the chromatin proteome in disease reveals remodeling principles and identifies high mobility group protein B2 as a regulator of hypertrophic growth. Mol Cell Proteomics, 11(6), M111 014258.

[105] Wang X, Andreassen PR, D'Andrea AD. (2004). Functional interaction of monoubiquitinated FANCD2 and BRCA2/FANCD1 in chromatin. Mol Cell Biol, 24(13), 5850-62.

[106] Chou DM, Adamson B, Dephoure NE, Tan X, Nottke AC, Hurov KE, . . . Elledge SJ. (2010). A chromatin localization screen reveals poly (ADP ribose)-regulated recruitment of the repressive 
polycomb and NuRD complexes to sites of DNA damage. Proc Natl Acad Sci U S A, 107(43), 1847580.

[107] Klein DC, Hainer SJ. (2020). Genomic methods in profiling DNA accessibility and factor localization. Chromosome Res, 28(1), 69-85.

[108] Mieczkowski J, Cook A, Bowman SK, Mueller B, Alver BH, Kundu S, . . Tolstorukov MY. (2016). MNase titration reveals differences between nucleosome occupancy and chromatin accessibility. Nat Commun, 7, 11485.

[109] Zaret K. (2005). Micrococcal nuclease analysis of chromatin structure. Curr Protoc Mol Biol, Chapter 21, Unit 211.

[110] Sanders MM. (1978). Fractionation of nucleosomes by salt elution from micrococcal nucleasedigested nuclei. J Cell Biol, 79(1), 97-109.

[111] Bloom KS, Anderson JN. (1978). Fractionation of hen oviduct chromatin into transcriptionally active and inactive regions after selective micrococcal nuclease digestion. Cell, 15(1), 141-50.

[112] Rocha E, Davie JR, van Holde KE, Weintraub H. (1984). Differential salt fractionation of active and inactive genomic domains in chicken erythrocyte. J Biol Chem, 259(13), 8558-63.

[113] Herrmann C, Avgousti DC, Weitzman MD. (2017). Differential Salt Fractionation of Nuclei to Analyze Chromatin-associated Proteins from Cultured Mammalian Cells. Bio Protoc, 7(6).

[114] Kustatscher G, Hegarat N, Wills KL, Furlan C, Bukowski-Wills JC, Hochegger H, Rappsilber J. (2014). Proteomics of a fuzzy organelle: interphase chromatin. EMBO J, 33(6), 648-64.

[115] Montagna RA, Wang TY. (1976). A comparison of the loosely bound and tightly bound nonhistone proteins from Ehrlich ascites tumor chromatin. Cancer Res, 36(9 pt.1), 3138-42.

[116] Kostraba NC, Montagna RA, Wang TY. (1975). Study of the loosely bound non-histone chromatin proteins. Stimulation of deoxyribonucleic acid-templated ribonucleic acid synthesis by a specific deoxyribonucleic acid-binding phosphoprotein fraction. J Biol Chem, 250(4), 1548-55.

[117] Phair RD, Scaffidi P, Elbi C, Vecerova J, Dey A, Ozato K, . . Misteli T. (2004). Global nature of dynamic protein-chromatin interactions in vivo: three-dimensional genome scanning and dynamic interaction networks of chromatin proteins. Mol Cell Biol, 24(14), 6393-402.

[118] Kustatscher G, Wills KL, Furlan C, Rappsilber J. (2014). Chromatin enrichment for proteomics. Nat Protoc, 9(9), 2090-9.

[119] Solomon MJ, Varshavsky A. (1985). Formaldehyde-mediated DNA-protein crosslinking: a probe for in vivo chromatin structures. Proc Natl Acad Sci U S A, 82(19), 6470-4.

[120] Kolb HC, Finn MG, Sharpless KB. (2001). Click Chemistry: Diverse Chemical Function from a Few Good Reactions. Angew Chem Int Ed Engl, 40(11), 2004-21.

[121] Mamidyala SK, Finn MG. (2010). In situ click chemistry: probing the binding landscapes of biological molecules. Chem Soc Rev, 39(4), 1252-61.

[122] Sirbu BM, Couch FB, Feigerle JT, Bhaskara S, Hiebert SW, Cortez D. (2011). Analysis of protein dynamics at active, stalled, and collapsed replication forks. Genes Dev, 25(12), 1320-7.

[123] Kliszczak AE, Rainey MD, Harhen B, Boisvert FM, Santocanale C. (2011). DNA mediated chromatin pull-down for the study of chromatin replication. Sci Rep, 1, 95.

[124] Aranda S, Alcaine-Colet A, Blanco E, Borras E, Caillot C, Sabido E, Di Croce L. (2019). Chromatin capture links the metabolic enzyme AHCY to stem cell proliferation. Sci Adv, 5(3), eaav2448. 
[125] Aranda S, Borràs E, Sabidó E, Di Croce L. (2020). Chromatin-Bound Proteome Profiling by Genome Capture. STAR Protocols, 100014.

[126] Bonev B, Cavalli G. (2016). Organization and function of the 3D genome. Nat Rev Genet, 17(11), $661-78$.

[127] Almouzni G, Cedar H. (2016). Maintenance of Epigenetic Information. Cold Spring Harb Perspect Biol, 8(5).

[128] Gilbert DM, Takebayashi SI, Ryba T, Lu J, Pope BD, Wilson KA, Hiratani I. (2010). Space and time in the nucleus: developmental control of replication timing and chromosome architecture. Cold Spring Harb Symp Quant Biol, 75, 143-53.

[129] Mirabella AC, Foster BM, Bartke T. (2016). Chromatin deregulation in disease. Chromosoma, 125(1), 75-93.

[130] Li X, Yu W, Qian X, Xia Y, Zheng Y, Lee JH, . . Lu Z. (2017). Nucleus-Translocated ACSS2 Promotes Gene Transcription for Lysosomal Biogenesis and Autophagy. Mol Cell, 66(5), 684-97 e9.

[131] Mews P, Donahue G, Drake AM, Luczak V, Abel T, Berger SL. (2017). Acetyl-CoA synthetase regulates histone acetylation and hippocampal memory. Nature, 546(7658), 381-6.

[132] Li X, Egervari G, Wang Y, Berger SL, Lu Z. (2018). Regulation of chromatin and gene expression by metabolic enzymes and metabolites. Nat Rev Mol Cell Biol, 19(9), 563-78.

[133] Sdelci S, Rendeiro AF, Rathert P, You W, Lin JG, Ringler A, . . Kubicek S. (2019). MTHFD1 interaction with BRD4 links folate metabolism to transcriptional regulation. Nat Genet, 51(6), 990-8.

[134] Erdel F, Rippe K. (2018). Formation of Chromatin Subcompartments by Phase Separation. Biophys J, 114(10), 2262-70. 
Figure 1. Principal chromatin-bound proteins that mediate chromatin topology and function. CR, chromatin remodeling; TF, transcription factor.

Figure 2. Influence of chromatin-bound proteins in human genetic disease. (a) A list of chromatin-bound proteins was established based on public protein databases and bibliographic research. Using the "chromatin" associated category, a list of potential chromatin-bound proteins of 2311 proteins from at least two different protein databases. Additionally, 2308 proteins were obtained from an extensive manual bibliographic search of previously published lists of chromatin regulators and well-established chromatin interactors (including TFs, histone writers and erasers, and ATP-remodelers). Merging of both gave a final list of 3097 known and potential chromatin-bound proteins. (b) The resultant percentage and absolute number of the 3097 identified chromatin-bound proteins (a) that were also in a list of: i) human proteins with a high intolerance to suffer loss-of-function mutations (Lek et al. 2016), ii) commonly identified cancer driver genes (Bailey et al. 2018, Lawrence', 2014, Martincorena, 2017, Dietlein`, 2020), and iii) the most commonly mutated genes in the PanCancer cohort (kandoth et al 2013).

Figure 3. Methods for global chromatin isolation from cells. Methods are classified as native chromatin isolation methods when the nucleus is obtained from living cells in native conditions, and as crosslinked chromatin isolation methods when the components of the nucleus have been chemically crosslinked. Dig., digestion. 
HETEROCHROMATIN
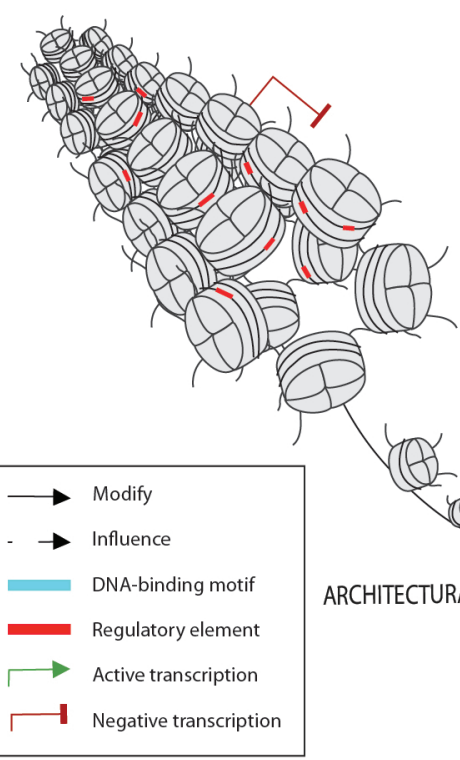

EUCHROMATIN

DNA loops

ARCHITECTURAL PROTEINS
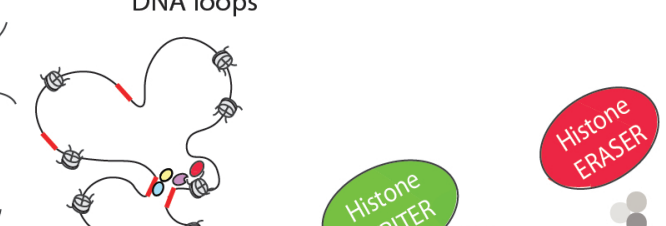

( His Whiter
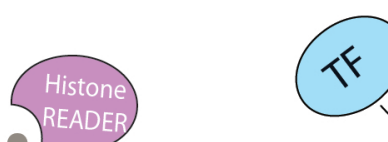

$-$
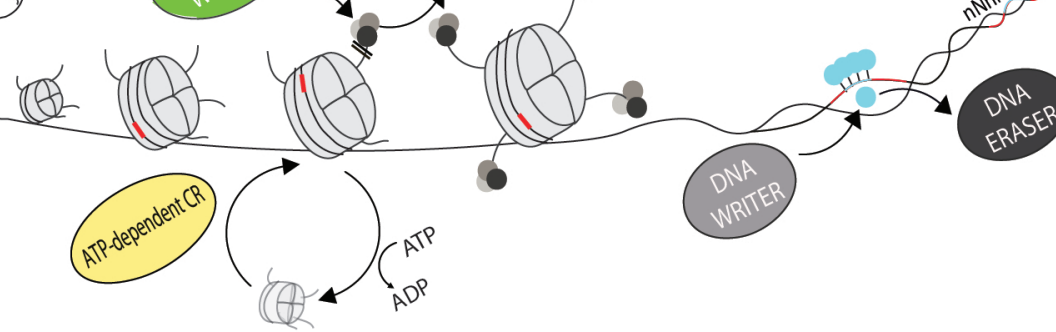


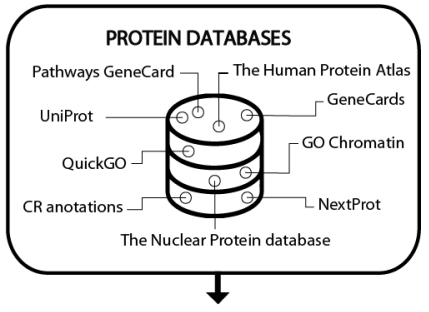

Proteins present

in at least two different data bases

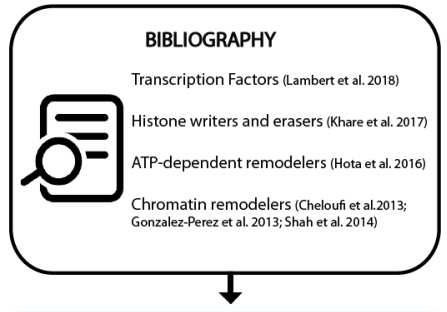

Proteins described as chromatin-bound proteins

\section{CHROMATIN-BOUND PROTEINS}

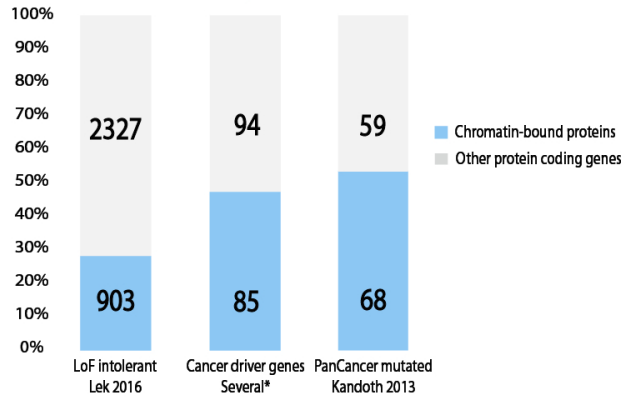




\section{Native chromatin isolation methods}

lonic detergent

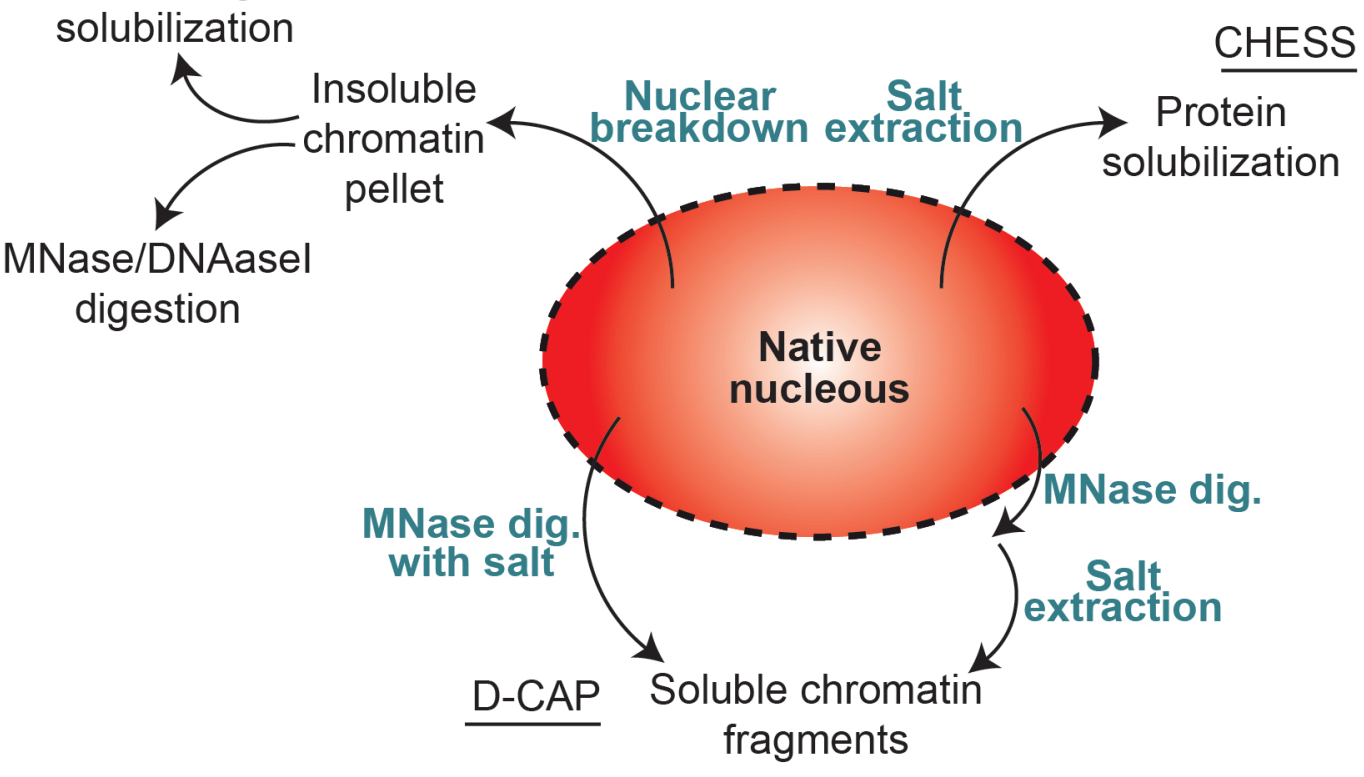

\section{Crosslinked chromatin isolation methods}

Gradient-seq
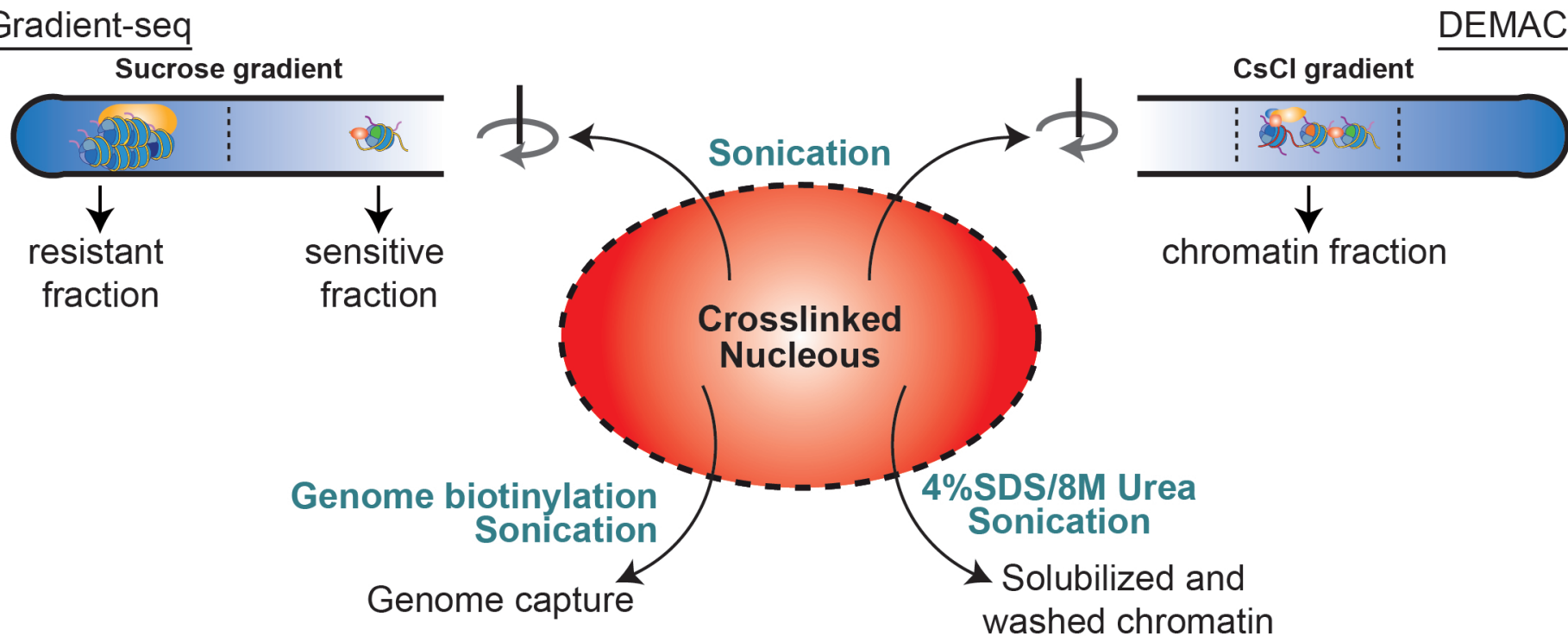
List of 3098 chromatin genes

Present in at least two categories of all the different consulted datasets*

1600027N09RIK

2410016O06RIK

*Consulted datasets

2410018M08RIK

Histone remodeleres (Khare et al. 017)

2900092E17RIK

Lambert et al. 2018

4931414P19RIK

Remodelers paper (Gonzalez-Perez et al. 2013; Cheloufi

ABCF2

ABRAXAS1

ATP-dependent chromatin remodelers paper (Hota et al

AC008770.3

UniProt

AC023509.3

QuickGO (Chromatin\&others)

AC092835.1

QuickGO GONAME (Chromatin\&others)

AC138696.1

GO_Chromatin

The human protein atlas

ACIN1

ACTA1

ACTB

ACTL6A

NextProt (Chromatin-associated proteins)

GeneCards (Chromatin)

Pathways Genecards

$\mathrm{CR}$ anotations

ACTL6B

The Nuclear protein database

ACTN4

ACTR5

ACTR6

ACTR8

ACTRT1

ADA

ADAR

ADNP

ADNP2

ADPRH

ADPRHL2

AEBP1

AEBP2

AFF4

$\mathrm{AHCY}$

AHCTF1

AHDC1

AHR

AHRR

AICDA

AIFM1

AIRE

AJUBA

AKAP1

AKAP8

AKAP8L

AKNA

ALKBH1

ALKBH2

ALKBH3

ALX1

ALX3

ALX4 
ANHX

ANKRD11

ANKRD17

ANKRD2

ANKRD31

ANKZF1

ANP32E

ANXA1

APBB1

APEX1

APLF

APOBEC1

APOBEC2

APOBEC3

APOBEC3B

APOBEC4

APTX

$A R$

ARGFX

ARHGAP35

ARID1A

ARID1B

ARID2

ARID3A

ARID3B

ARID3C

ARID4A

ARID4B

ARID5A

ARID5B

ARIH2

ARNT

ARNT2

ARNTL

ARNTL2

ARRB1

ART1

ARX

ASAP1

ASCL1

ASCL2

ASCL3

ASCL4

ASCL5

ASF1A

ASF1B

ASH1L

ASH2L

ASXL1

ASXL2

ASXL3 
ATAD2

ATAD2B

ATAT1

ATF1

ATF2

ATF3

ATF4

ATF5

ATF6

ATF6B

ATF7

ATF7IP

ATF7IP2

ATM

ATMIN

ATOH1

ATOH7

ATOH8

ATR

ATRX

ATXN3

ATXN7

ATXN7L3

AURKA

AURKB

AUTS2

BABAM1

BABAM2

$\mathrm{BACH} 1$

$\mathrm{BACH} 2$

BAG6

BAHCC1

BAHD1

BANF1

BANP

BAP1

BAP18

BARHL1

BARHL2

BARX1

BARX2

BATF

BATF2

BATF3

BAZ1A

BAZ1B

BAZ2A

BAZ2B

$B B X$

BCAS3

BCL11A 
BCL11B

BCL6

BCL6B

BCOR

BCORL1

BDP1

BEND3

BEND6

BHLHA15

BHLHA9

BHLHE22

BHLHE23

BHLHE40

BHLHE41

BICRA

BICRAL

BLM

BMI1

BNC1

BNC2

BOP1

BORCS8-MEF2B

BPTF

BRCA1

BRCA2

BRCC3

BRD1

BRD2

BRD3

BRD4

BRD7

BRD8

BRD9

BRDT

BRE

BRF2

BRIP1

BRMS1

BRMS1L

BRPF1

BRPF3

BRWD1

BRWD3

BSX

BTAF1

BUB3

BUD23

BUD31

C11orf95

C14orf169

C14orf93 
C17orf49

C17orf96

CABIN1

CALCOCO1

CAMK4

CAMKMT

CAMTA1

CAMTA2

CAND1

CAPN2

CARF

CARM1

CASZ1

CBFA2T2

CBFA2T3

CBX1

CBX2

CBX3

CBX4

CBX5

CBX6

CBX7

CBX8

CC2D1A

CCAR2

CCDC101

CCDC169-SOHLH2

CCDC17

CCNA1

CCND2

CCNT1

CCNT2

CDA

CDAN1

CDC45

CDC5L

CDC6

CDC73

CDCA2

CDCA5

CDCA7L

CDCA8

CDK1

CDK12

CDK13

CDK2AP1

CDK4

CDK5

CDK9

CDKN2A

CDT1 
CDX1

CDX2

CDX4

CDY1

CDY1B

CDY2A

CDY2B

CDYL

CDYL2

CEBPA

CEBPB

CEBPD

CEBPE

CEBPG

CEBPZ

CECR2

CENPA

CENPB

CENPBD1

CENPC

CENPF

CENPH

CENPI

CENPK

CENPL

CENPM

CENPN

CENPO

CENPP

CENPQ

CENPS

CENPT

CENPU

CENPV

CENPW

CENPX

CEP164

CGAS

CGGBP1

CHAF1A

CHAF1B

CHAMP1

CHCHD3

CHD1

CHD1L

CHD2

CHD3

CHD4

CHD5

CHD6

CHD7 
CHD8

CHD9

CHEK1

CHEK2

CHMP1A

CHMP1B

CHMP2A

CHMP2B

CHMP4A

CHMP4B

CHMP4C

CHMP5

CHMP6

CHMP7

CHRAC1

CHTF18

CHTF8

CHTOP

CHUK

$\mathrm{CIC}$

CINP

CITED1

CITED2

CKS2

CLOCK

COPRS

COPS3

COPS4

COPS5

COPS6

COPS9

COQ7

CPEB1

CPHXL

CPSF6

CPXCR1

CRAMP1

CREB1

CREB3

CREB3L1

CREB3L2

CREB3L3

CREB3L4

CREB5

CREBBP

CREBL2

CREBZF

CREM

CRTC2

CRX

CSNK2A1 
CSNK2A2

CSNK2B

CSNKA2IP

CSRNP1

CSRNP2

CSRNP3

CTBP1

CTBP2

CTCF

CTCFL

CTDSPL2

CTNNB1

CTR9

CTSL

CUL1

CUL4B

CUX1

CUX2

CXXC1

CXXC4

CXXC5

D14ERTD668E

DACH1

$\mathrm{DACH} 2$

DAPK3

DAXX

DBF4B

DBP

DBX1

DBX2

DCAF1

DDB1

DDB2

DDIT3

DDX1

DDX11

DDX17

DDX21

DDX23

DDX3X

DDX41

DDX5

DDX6

DEAF1

DEK

DFFA

DFFB

DGCR8

DHX29

DHX30

DHX36 
DHX9

DIDO1

DKC1

DLD

DLST

DLX1

DLX2

DLX3

DLX4

DLX5

DLX6

DMAP1

DMBX1

DMRT1

DMRT2

DMRT3

DMRTA1

DMRTA2

DMRTB1

DMRTC1

DMRTC1B

DMRTC2

DMTF1

DNAJC2

DNASE1L3

DNMT1

DNMT3A

DNMT3B

DNMT3L

DNTT

DNTTIP1

DNTTIP2

DOT1L

DPF1

DPF2

DPF3

DPPA2

DPPA3

DPPA4

DPRX

DPY30

DR1

DRAP1

DRGX

DSCC1

DTD1

DTX3L

DUB2A

DUX1

DUX3

DUX4 
DUXA

DVL3

DZIP1

DZIP3

E130308A19RIK

E2F1

E2F2

E2F3

E2F4

E2F5

E2F6

E2F7

E2F8

E4F1

EBF1

EBF2

EBF3

EBF4

EDF1

EEA1

EED

EG330129

EG546387

EGFR

EGR1

EGR2

EGR3

EGR4

EHF

EHMT1

EHMT2

EID1

EIF3E

EIF4A3

ELF1

ELF2

ELF3

ELF4

ELF5

ELK1

ELK3

ELK4

ELL

ELOB

ELOF1

ELP1

ELP2

ELP3

ELP4

EMD

EME1 
EME2

EMG1

EMSY

EMX1

EMX2

EN1

EN2

ENC1

ENY2

EOMES

EP300

EP400

EPAS1

EPC1

EPC2

EPOP

ERCC1

ERCC3

ERCC4

ERCC5

ERCC6

ERCC6L

ERF

ERG

ESCO1

ESCO2

ESR1

ESR2

ESRRA

ESRRB

ESRRG

ESX1

ETS1

ETS2

ETV1

ETV2

ETV3

ETV3L

ETV4

ETV5

ETV6

ETV7

EVX1

EVX2

EWSR1

EXD2

EXO1

EXOSC10

EXOSC3

EXOSC4

EXOSC5 
EXOSC9

EYA1

EYA2

EYA3

EYA4

EZH1

EZH2

FAAP24

FABP1

FACT

FAF1

FAM111A

FAM170A

FAM175A

FAM175B

FAM200B

FANCM

FANK1

FBH1

FBL

FBXL19

FBXO17

FBXO44

FBXW9

FEM1B

FER

FERD3L

FEV

FEZF1

FEZF2

FIGLA

FIZ1

FKBP1A

FKBP2

FKBP5

FLI1

FLNA

FLYWCH1

FMR1

FNBP4

FOS

FOSB

FOSL1

FOSL2

FOXA1

FOXA2

FOXA3

FOXB1

FOXB2

FOXC1

FOXC2 
FOXD1

FOXD2

FOXD3

FOXD4

FOXD4L1

FOXD4L3

FOXD4L4

FOXD4L5

FOXD4L6

FOXE1

FOXE3

FOXF1

FOXF2

FOXG1

FOXH1

FOXI1

FOXI2

FOXI3

FOXJ1

FOXJ2

FOXJ3

FOXK1

FOXK2

FOXL1

FOXL2

FOXM1

FOXN1

FOXN2

FOXN3

FOXN4

FOXO1

FOXO3

FOXO4

FOXO6

FOXP1

FOXP2

FOXP3

FOXP4

FOXO1

FOXR1

FOXR2

FOXS1

FSHR

FTSJ3

FUBP3

FUS

FXR1

FXR2

G2E3

GABPA

GADD45A 
GADD45B

GATA1

GATA2

GATA3

GATA4

GATA5

GATA6

GATAD1

GATAD2A

GATAD2B

GBX1

GBX2

GCM1

GCM2

GFI1

GFI1B

GLI1

GLI2

GLI3

GLI4

GLIS1

GLIS2

GLIS3

GLMP

GLYR1

GM4349

GMEB1

GMEB2

GMNC

GMNN

GPBP1

GPBP1L1

GPER1

GPS2

GPX4

GRHL1

GRHL2

GRHL3

GRWD1

GSC

GSC2

GSG2

GSX1

GSX2

GTF2B

GTF2F1

GTF2H1

GTF2I

GTF2IRD1

GTF2IRD2

GTF2IRD2B 
GTF3A

GTF3C1

GTF3C4

GTF3C5

GZF1

H1-0

H1-1

H1-10

H1-2

H1-3

H1-4

H1-5

H1-6

H1-7

H1-8

H1F0

H1FNT

H1FOO

H1FX

H2AB1

H2AB2

$\mathrm{H} 2 \mathrm{AB} 3$

H2AC1

H2AC11

$\mathrm{H} 2 \mathrm{AC} 12$

$\mathrm{H} 2 \mathrm{AC} 13$

$\mathrm{H} 2 \mathrm{AC} 14$

$\mathrm{H} 2 \mathrm{AC} 15$

H2AC16

H2AC17

H2AC18

H2AC19

$\mathrm{H} 2 \mathrm{AC} 20$

H2AC21

H2AC4

H2AC6

$\mathrm{H} 2 \mathrm{AC} 7$

H2AC8

H2AFB1

H2AFB2

H2AFB3

H2AFJ

H2AFV

H2AFX

H2AFY

H2AFY2

H2AFZ

H2AJ

H2AP

H2AW

H2AX 
H2AZ1

H2AZ2

$\mathrm{H} 2 \mathrm{BC} 1$

H2BC10

H2BC11

$\mathrm{H} 2 \mathrm{BC} 12$

$\mathrm{H} 2 \mathrm{BC} 13$

$\mathrm{H} 2 \mathrm{BC} 14$

H2BC15

H2BC17

H2BC18

H2BC21

$\mathrm{H} 2 \mathrm{BC} 3$

$\mathrm{H} 2 \mathrm{BC} 4$

H2BC5

H2BC6

$\mathrm{H} 2 \mathrm{BC} 7$

$\mathrm{H} 2 \mathrm{BC} 8$

H2BC9

H2BFM

H2BFS

H2BFWT

H2BU1

H2BW2

H3-3A

H3-3B

H3-5

H3C1

H3C10

H3C11

H3C12

$\mathrm{H} 3 \mathrm{C} 13$

$\mathrm{H} 3 \mathrm{C} 14$

H3C15

H3C2

$\mathrm{H} 3 \mathrm{C} 3$

$\mathrm{H} 3 \mathrm{C} 4$

H3C6

$\mathrm{H} 3 \mathrm{C} 7$

$\mathrm{H} 3 \mathrm{C} 8$

H3F3A

H3F3B

H3F3C

H4-16

H4C1

$\mathrm{H} 4 \mathrm{C} 11$

$\mathrm{H} 4 \mathrm{C} 12$

$\mathrm{H} 4 \mathrm{C} 13$

$\mathrm{H} 4 \mathrm{C} 14$

H4C15

$\mathrm{H} 4 \mathrm{C} 2$ 
$\mathrm{H} 4 \mathrm{C} 3$

$\mathrm{H} 4 \mathrm{C} 4$

$\mathrm{H} 4 \mathrm{C} 5$

$\mathrm{H} 4 \mathrm{C} 6$

$\mathrm{H} 4 \mathrm{C} 8$

H4C9

HAND1

HAND2

HASPIN

HAT1

HBP1

HCFC1

HCFC2

HDAC1

HDAC10

HDAC11

HDAC2

HDAC3

HDAC4

HDAC5

HDAC6

HDAC7

HDAC8

HDAC9

HDGF

HDGFL1

HDGFL2

HDGFRP2

HDGFRP3

HDLBP

HDX

HEATR1

HELB

HELLS

HELT

HES1

HES2

HES3

HES4

HES5

HES6

HES7

HESX1

HEY1

HEY2

HEYL

HHEX

HIC1

HIC2

HIF1A

HIF3A 
HILS1

HINFP

HIPK1

HIRA

HIRIP3

HIST1H1A

HIST1H1B

HIST1H1C

HIST1H1D

HIST1H1E

HIST1H1T

HIST1H2AA

HIST1H2AB

HIST1H2AC

HIST1H2AD

HIST1H2AE

HIST1H2AG

HIST1H2AH

HIST1H2AI

HIST1H2AJ

HIST1H2AK

HIST1H2AL

HIST1H2AM

HIST1H2BA

HIST1H2BB

HIST1H2BC

HIST1H2BD

HIST1H2BE

HIST1H2BF

HIST1H2BG

HIST1H2BH

HIST1H2BI

HIST1H2BJ

HIST1H2BK

HIST1H2BL

HIST1H2BM

HIST1H2BN

HIST1H2BO

HIST1H3A

HIST1H3B

HIST1H3C

HIST1H3D

HIST1H3E

HIST1H3F

HIST1H3G

HIST1H3H

HIST1H3I

HIST1H3J

HIST1H4A

HIST1H4B

HIST1H4C 


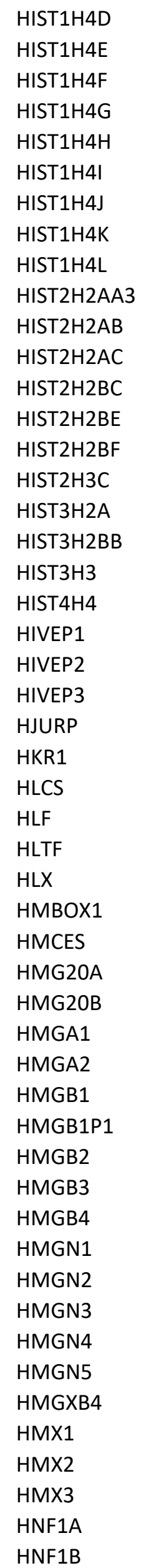


HNF4A

HNF4G

HNRNPAO

HNRNPA1

HNRNPA2B1

HNRNPC

HNRNPD

HNRNPF

HNRNPK

HNRNPL

HNRNPM

HNRNPR

HNRNPU

HNRNPUL1

HOMEZ

HOPX

HORMAD1

HOXA1

HOXA10

HOXA11

HOXA13

HOXA2

HOXA3

HOXA4

HOXA5

HOXA6

HOXA7

HOXA9

HOXB1

HOXB13

HOXB2

HOXB3

HOXB4

HOXB5

HOXB6

HOXB7

HOXB8

HOXB9

HOXC10

HOXC11

HOXC12

HOXC13

HOXC4

HOXC5

HOXC6

HOXC8

HOXC9

HOXD1

HOXD10

HOXD11

HOXD12 
HOXD13

HOXD3

HOXD4

HOXD8

HOXD9

HP1BP3

HPRT

HR

HSF1

HSF2

HSF4

HSF5

HSFX1

HSFX2

HSFX3

HSFY1

HSFY2

HTRA2

HUWE1

HYPM

ICE1

ICE2

IFI16

IFT74

IGF2BP3

IKBKAP

IKZF1

IKZF2

IKZF3

IKZF4

IKZF5

IL33

ILF2

ILF3

INCENP

ING1

ING2

ING3

ING4

ING5

INO80

INO80B

INO80C

INO80D

INO80E

INSM1

INSM2

INTS12

IPO4

IRF1

IRF2 
IRF3

IRF4

IRF5

IRF6

IRF7

IRF8

IRF9

IRX1

IRX2

IRX3

IRX4

IRX5

IRX6

ISL1

ISL2

IST1

ISX

ITGB3BP

IWS1

JADE1

JADE2

JADE3

JAK2

JARID2

JAZF1

JDP2

JHDM1D

JMJD1C

JMJD4

JMJD5

JMJD6

JMJD8

JRK

JRKL

JUN

JUNB

JUND

KANSL1

KANSL2

KANSL3

KAT2A

KAT2B

KAT5

KAT6A

KAT6B

KAT7

KAT8

KCMF1

KCNIP3

KDM1A

KDM1B 
KDM2A

KDM2B

KDM3A

KDM3B

KDM4A

KDM4B

KDM4C

KDM4D

KDM4DL

KDM4E

KDM5A

KDM5B

KDM5C

KDM5D

KDM6A

KDM6B

KDM7A

KDM8

KHDRBS1

KIAA1045

KIAA1958

KIAA2026

KIF22

KIN

KLF1

KLF10

KLF11

KLF12

KLF13

KLF14

KLF15

KLF16

KLF17

KLF2

KLF3

KLF4

KLF5

KLF6

KLF7

KLF8

KLF9

KLHDC3

KMT2A

KMT2B

KMT2C

KMT2D

KMT2E

KMT5A

KMT5B

KMT5C

KNL1 
L3MBTL

L3MBTL1

L3MBTL2

L3MBTL3

L3MBTL4

LAS1L

LBR

LBX1

LBX2

LCOR

LCORL

LDB1

LEF1

LEMD2

LEUTX

LHX1

LHX2

LHX3

LHX4

LHX5

LHX6

LHX8

LHX9

LIG4

LIN28A

LIN28B

LIN54

LIN9

LMNA

LMNB1

LMNB2

LMX1A

LMX1B

LNX1

LOC100040412

LOC100044324

LOC100048887

LOC100129278

LOC664892

LOXL2

LRWD1

LTF

LYL1

M1AP

MACROH2A1

MACROH2A2

MAEA

MAEL

MAF

MAFA

MAFB 
MAFF

MAFG

MAFK

MAGED1

MAML1

MAP1S

MAP3K12

MAPK1

MAPK15

MAPKAPK3

MATR3

MAU2

MAX

MAZ

MBD1

$M B D 2$

MBD3

MBD3L1

MBD3L2

MBD3L3

MBD3L4

MBD3L5

MBD4

MBD5

MBD6

MBIP

MBNL2

MBTD1

MCM10

MCM2

$\mathrm{MCM} 3$

MCMЗАP

MCM4

MCM5

MCM6

MCM7

MCM8

MCM9

MCMBP

MCRS1

MDC1

MEAF6

MECOM

MECP2

MED1

MED12

MED25

MED26

MED6

MEF2A

MEF2B 
MEF2C

MEF2D

MEIOB

MEIS1

MEIS2

MEIS3

MEN1

MEOX1

MEOX2

MEPCE

MESP1

MESP2

METTL21D

METTL8

MGA

MGEA5

MGMT

MIER1

MINA

MIS12

MIS18A

MIS18BP1

MITF

MIXL1

MIZF

MKI67

MKX

MLH1

MLH3

MLL

MLL1

MLL2

MLL3

MLL4

MLL5

MLLT1

MLLT10

MLLT3

MLLT6

MLX

MLXIP

MLXIPL

MNDA

MNT

MNX1

MORC2

MORF4L1

MORF4L2

MOS

MPG

MPHOSPH8 
MPO

MRE11

MRGBP

MRNIP

MSANTD1

MSANTD3

MSANTD4

MSC

MSGN1

$\mathrm{MSH} 2$

MSH6

MSL1

MSL2

MSL3

MSL3L1

MSX1

MSX2

MTA1

MTA2

MTA3

MTBP

MTERF

MTERF1

MTERF2

MTERF3

MTERF4

MTF1

MTF2

MTHFR

MTHFD1

MTR

MTREX

MUC1

MUM1

MUM1L1

MXD1

MXD3

MXD4

MXI1

MXRA8

MYB

MYBBP1A

MYBL1

MYBL2

MYC

MYCL

MYCN

MYEF2

MYF5

MYF6

MYNN 
MYO1C

MYOCD

MYOD1

MYOG

MYPOP

MYRF

MYRFL

MYSM1

MYST1

MYST2

MYST3

MYST4

MYT1

MYT1L

MZF1

N28178

NAA10

NAA15

NAA60

NACC1

NACC2

NAIF1

NANOG

NANOGNB

NANOGP8

NAP1L1

NAP1L2

NAP1L3

NAP1L4

NASP

NAT10

NAT6

NBN

NCAPD2

NCAPD3

NCAPG

$\mathrm{NCAPH}$

NCAPH2

$\mathrm{NCL}$

NCOA1

NCOA2

NCOA3

NCOA5

NCOA6

NCOR1

NCOR1P1

NCOR2

NEDD4

NEDD4L

NEIL1

NEK6 
NEK9

NELFA

NELFB

NELFCD

NELFE

NEUROD1

NEUROD2

NEUROD4

NEUROD6

NEUROG1

NEUROG2

NEUROG3

NFAT5

NFATC1

NFATC2

NFATC3

NFATC4

NFE2

NFE2L1

NFE2L2

NFE2L3

NFE4

NFIA

NFIB

NFIC

NFIL3

NFIX

NFKB1

NFKB2

NFRKB

NFX1

NFXL1

NFYA

NFYB

NFYC

NHLH1

NHLH2

NHP2

NIPBL

NKAP

NKAPL

NKRF

NKX1-1

NKX1-2

NKX2-1

NKX2-2

NKX2-3

NKX2-4

NKX2-5

NKX2-6

NKX2-8 
NKX3-1

NKX3-2

NKX6-1

NKX6-2

NKX6-3

NME2

N066

NOBOX

$\mathrm{NOC2L}$

NOC3L

NOLC1

NONO

NOP53

NOP56

NOP58

NOTCH1

NOTO

NPAS1

NPAS2

NPAS3

NPAS4

NPM1

NPM2

NPM3

NROB1

NROB2

NR1D1

NR1D2

$\mathrm{NR} 1 \mathrm{H} 2$

NR1H3

$\mathrm{NR} 1 \mathrm{H} 4$

NR1/2

NR1I3

NR2C1

NR2C2

NR2E1

NR2E3

NR2F1

NR2F2

NR2F6

NR3C1

NR3C2

NR4A1

NR4A2

NR4A3

NR5A1

NR5A2

NR6A1

NRDE2

NRF1

NRIP1 
NRL

NSD1

NSD2

NSD3

NSMF

NUCKS1

NUDT16L1

NUDT21

NUDT5

NUFIP1

NUP107

NUP133

NUP155

NUP160

NUP205

NUP62

NUP85

NUP98

NUPR1

NUSAP1

OBI1

OGT

OIP5

OLIG1

OLIG2

OLIG3

ONECUT1

ONECUT2

ONECUT3

ORC1

ORC2

ORC3

ORC4

ORC5

OSR1

OSR2

OTP

OTX1

OTX2

OVOL1

OVOL2

OVOL3

p53

PA2G4

PABPC1L

PADI1

PADI2

PADI3

PADI4

PADI6

PAF1 
PAG1

PAGR1

PAK1

PALB2

PARG

PARK7

PARP1

PARP10

PARP11

PARP12

PARP14

PARP16

PARP2

PARP3

PARP4

PARP6

PARP8

PARP9

PARPBP

PATZ1

PAWR

PAX1

PAX2

PAX3

PAX4

PAX5

PAX6

PAX7

PAX8

PAX9

PAXBP1

PAXIP1

PBRM1

PBX1

PBX2

PBX3

PBX4

PCGF1

PCGF2

PCGF6

PCID2

PCLAF

PCMT1

PCNA

PDS5A

PDS5B

PDX1

PEG3

PELP1

PER1

PGR 
PHB

PHC1

PHC2

PHC3

PHF1

PHF10

PHF11

PHF12

PHF13

PHF14

PHF15

PHF16

PHF17

PHF19

PHF2

PHF20

PHF20L1

PHF21A

PHF21B

PHF23

PHF3

PHF5A

PHF6

PHF7

PHF8

PHIP

PHOX2A

PHOX2B

PHRF1

PIAS1

PIH1D1

PIN1

PINK1

PITX1

PITX2

PITX3

PIWIL1

PKN1

PKNOX1

PKNOX2

PLAC8

PLAG1

PLAGL1

PLAGL2

PLCB1

PLK1

PLK2

PLSCR1

PML

POGK

POGZ 
POLA1

POLD1

POLE

POLE3

POLG

POLH

POLQ

POLR1A

POLR2A

POLR2B

POLR2E

POLR3A

POLR3D

POLR3G

POLR3GL

POU1F1

POU2AF1

POU2F1

POU2F2

POU2F3

POU3F1

POU3F2

POU3F3

POU3F4

POU4F1

POU4F2

POU4F3

POU5F1

POU5F1B

POU5F2

POU6F1

POU6F2

PPARA

PPARD

PPARG

PPARGC1A

PPHLN1

PPIB

PPM1D

PPP1CA

PPP1CB

PPP1CC

PPP1R10

PPP3CA

PPP4C

PRDM1

PRDM10

PRDM11

PRDM12

PRDM13

PRDM14 
PRDM15

PRDM16

PRDM2

PRDM4

PRDM5

PRDM6

PRDM7

PRDM8

PRDM9

PREB

PRIMPOL

PRKAA1

PRKAA2

PRKCB

PRKCD

PRKDC

PRM1

PRM2

PRM3

PRMT1

PRMT2

PRMT3

PRMT5

PRMT6

PRMT7

PRMT8

PROP1

PROX1

PROX2

PRPF19

PRPF3

PRPF4

PRR12

PRR14

PRRC2B

PRRX1

PRRX2

PSD3

PSIP1

PSMA2

PSMA4

PSMA5

PSMA6

PSMA7

PSMB1

PSMB3

PSMB4

PSMB5

PSMB6

PSMC1

PSMC2 
PSMC3

PSMC4

PSMC5

PSMC6

PSMD1

PSMD11

PSMD12

PSMD13

PSMD14

PSMD2

PSMD3

PSMD4

PSMD6

PSMD7

PSMD8

PSME4

PTF1A

PTMA

PTPN2

PURA

PURB

PURG

PWP1

PWWP2A

PWWP2B

PWWP3A

PYGO1

PYGO2

Q6ZW69

RAD17

RAD18

RAD21

RAD21L1

RAD23B

RAD50

RAD51

RAD51AP1

RAD54L

RAE1

RAG1

RAG2

RAI1

RAN

RANBP1

RANBP2

RANGAP1

RARA

RARB

RARG

RAX

RAX2 
RB1

RBAK

RBBP4

RBBP5

RBBP7

RBBP8

RBCK1

RBL1

RBL2

RBM10

RBMX

RBPJ

RBPJL

RBSN

RC3H1

RCBTB1

RCC1

RCCD1

RCOR1

REC8

REL

RELA

RELB

Remodeleres

REPIN1

RERE papers (Nuria's+Sł papers)

RESF1

REST

REV3L

REXO4

RFC1

RFC2

RFC3

RFWD2

RFX1

RFX2

RFX3

RFX4

RFX5

RFX6

RFX7

RFX8

RFXANK

RFXAP

RHOXF1

RHOXF2

RHOXF2B

RIF1

RING1

RIOX1

RIOX2 
RIT2

RLF

RNF168

RNF169

RNF17

RNF2

RNF20

RNF217

RNF40

RNF8

RORA

RORB

RORC

RPA1

RPA2

RPA3

RPL26

RPL3

RPS19BP1

RPS2

RPS6KA3

RPS6KA4

RPS6KA5

RREB1

RRP1B

RRP8

RSBN1

RSF1

RTF1

RUNX1

RUNX1T1

RUNX2

RUNX3

RUVBL1

RUVBL2

RXRA

RXRB

RXRG

RYBP

SAFB

SAFB2

SALL1

SALL2

SALL3

SALL4

SAP130

SAP18

SAP30

SAP30L

SART1

SATB1 
SATB2

SBNO1

SCMH1

SCML2

SCML4

SCRT1

SCRT2

SCX

SDR16C5

SEBOX

SEH1L

SENP2

SENP3

SERBP1

SET

SETBP1

SETD1A

SETD1B

SETD2

SETD3

SETD4

SETD5

SETD6

SETD7

SETD8

SETD9

SETDB1

SETDB2

SETMAR

SETSIP

SETX

SF3A1

SF3B1

SF3B2

SF3B3

SFMBT1

SFMBT2

SFPQ

SGF29

SGSM2

SHMT2

SHOX

SHOX2

SHPRH

SIAH2

SIK1

SIM1

SIM2

SIN3A

SIN3B

SINHCAF 
SIRT1

SIRT2

SIRT3

SIRT4

SIRT5

SIRT6

SIRT7

SIX1

SIX2

SIX3

SIX4

SIX5

SIX6

SKI

SKIL

SKOR1

SKOR2

SKP1

SKP2

SLC2A4RG

SLC30A9

SLF1

SLF2

SLK

SLX4

SMAD1

SMAD2

SMAD3

SMAD4

SMAD5

SMAD6

SMAD7

SMAD9

SMARCA1

SMARCA2

SMARCA4

SMARCA5

SMARCAD1

SMARCAL1

SMARCB1

SMARCC1

SMARCC2

SMARCD1

SMARCD2

SMARCD3

SMARCE1

SMC1A

SMC1B

SMC2

SMC3

SMC4 
SMC5

SMC6

SMCHD1

SMCR8

SMN1

SMN2

SMNDC1

SMYD1

SMYD2

SMYD3

SMYD4

SMYD5

SNAI1

SNAI2

SNAI3

SNAPC2

SNAPC4

SNAPC5

SND1

SNRPD2

SNW1

SOHLH1

SOHLH2

SON

SOS1

SOS2

SOX1

SOX10

SOX11

SOX12

SOX13

SOX14

SOX15

SOX17

SOX18

SOX 2

SOX21

SOX3

SOX30

SOX4

SOX5

Sox6

SOX7

SOX8

SOX9

SP1

SP100

SP110

SP140

SP140L

SP2 
SP3

SP4

SP5

SP6

SP7

SP8

SP9

SPATA24

SPDEF

SPEN

SPHK2

SPI1

SPIB

SPIC

SPIN1

SPINDOC

SPTY2D1

SPZ1

SRBD1

SRCAP

SREBF1

SREBF2

SRF

SRPK1

SRSF2

SRY

SS18

SS18L1

SSBP1

SSRP1

SSU72

ST18

STAG1

STAG2

STAG3

STAG3L1

STAG3L2

STAG3L3

STAG3L4

STAT1

STAT2

STAT3

STAT4

STAT5A

STAT5B

STAT6

STIP1

STK3

STK31

STK4

STPG4 
SUB1

SUDS3

SUMO1

SUMO2

SUMO3

SUPT16H

SUPT3H

SUPT4H1

SUPT5H

SUPT6H

SUPT7L

SUPV3L1

SUV39H1

SUV $39 \mathrm{H} 2$

SUV42OH1

SUV42OH2

SUZ12

SVEP1

SYCE1

SYCE2

SYCP1

SYCP3

SYNCRIP

$T$

TADA1

TADA2A

TADA2B

TADA3

TAF1

TAF10

TAF12

TAF15

TAF1L

TAF2

TAF3

TAF4

TAF5

TAF5L

TAF6L

TAF9

TAF9B

TAL1

TAL2

TARDBP

TASOR

TBL1X

TBL1XR1

TBP

TBPL1

TBPL2

TBR1 
TBX1

TBX10

TBX15

TBX18

TBX19

TBX2

TBX20

TBX21

TBX22

TBX3

TBX4

TBX5

TBX6

TBXT

TCERG1

TCF12

TCF15

TCF19

TCF20

TCF21

TCF23

TCF24

TCF3

TCF4

TCF7

TCF7L1

TCF7L2

TCFL5

TCOF1

TCP1

TDG

TDRD1

TDRD10

TDRD12

TDRD3

TDRD5

TDRD6

TDRD7

TDRD9

TDRKH

TEAD1

TEAD2

TEAD3

TEAD4

TEF

TERB1

TERF1

TERF2

TERF2IP

TET1

TET2 
TET3

TEX10

TFAM

TFAP2A

TFAP2B

TFAP2C

TFAP2D

TFAP2E

TFAP4

TFCP2

TFCP2L1

TFDP1

TFDP2

TFDP3

TFE3

TFEB

TFEC

TFPT

TGIF1

TGIF2

TGIF2LX

TGIF2LY

THAP1

THAP10

THAP11

THAP12

THAP2

THAP3

THAP4

THAP5

THAP6

THAP7

THAP8

THAP9

THRA

THRAP3

THRB

THYN1

TICRR

TIGD1

TIGD2

TIGD3

TIGD4

TIGD5

TIGD6

TIGD7

TIMELESS

TIPARP

TIPIN

TLE1

TLE4 
TLK1

TLK2

TLX1

TLX2

TLX3

TMF1

TMPO

TNKS1BP1

TNP1

TNP2

TNRC18

TOP1

TOP2A

TOP2B

TOPORS

TOX

TOX3

TOX4

TP53

TP53BP1

TP63

TP73

TPR

TPRX1

TPX2

TRAFD1

TRDMT1

TRERF1

TRIM24

TRIM28

TRIM33

TRIM37

TRIM5

TRIM66

TRIP12

TRNP1

TRP53BP1

TRPS1

TRRAP

TSC22D1

TSHZ1

TSHZ2

TSHZ3

TSPYL2

TSSK6

TTC21B

TTC37

TTC5

TTF1

TTF2

TUBA1A 
TUBA1B

TWIST1

TWIST2

UBA1

UBA52

UBA7

UBB

UBE2A

UBE2B

UBE2C

UBE2E1

UBE2E3

UBE2I

UBE2M

UBE2N

UBE2O

UBE2Q2

UBE2T

UBE2U

UBE3A

UBE4A

UBN1

UBP1

UBR1

UBR2

UBR3

UBR4

UBR5

UBR7

UBTF

UCHL5

UHRF1

UHRF2

UIMC1

UNCX

UPF1

URI1

USF1

USF2

USF3

USP11

USP12

USP13

USP16

USP21

USP22

USP27X

USP3

USP34

USP35

USP46 
USP49

USP51

USP7

UTF1

UTP15

UTP3

UTP4

UTY

UXT

VAX1

VAX2

VCP

VCX

VDR

VENTX

VEZF1

VIRMA

VPS72

VRK1

VRK2

VSX1

VSX2

WAC

WAPL

WBP2

WBP2NL

WBP7

WDHD1

WDR13

WDR18

WDR43

WDR5

WDR61

WDR77

WDR82

WHSC1

WHSC1L1

WIZ

WNT5A

WRN

WT1

WTAP

XBP1

XPA

XPC

XPO1

XRCC1

XRCC5

XRCC6

YAF2

YAP1 
YBX1

YBX2

YBX3

YEATS2

YEATS4

YTHDC1

YWHAQ

YY1

YY1AP1

YY2

ZBED1

ZBED2

ZBED3

ZBED4

ZBED5

ZBED6

ZBED9

ZBTB1

ZBTB10

ZBTB11

ZBTB12

ZBTB14

ZBTB16

ZBTB17

ZBTB18

ZBTB2

ZBTB20

ZBTB21

ZBTB22

ZBTB24

ZBTB25

ZBTB26

ZBTB3

ZBTB32

ZBTB33

ZBTB34

ZBTB37

ZBTB38

ZBTB39

ZBTB4

ZBTB40

ZBTB41

ZBTB42

ZBTB43

ZBTB44

ZBTB45

ZBTB46

ZBTB47

ZBTB48

ZBTB49

ZBTB5 
ZBTB6

ZBTB7A

ZBTB7B

ZBTB7C

ZBTB8A

ZBTB8B

ZBTB9

ZC3H12A

$\mathrm{ZC} 3 \mathrm{H} 4$

ZC3H6

ZC3H8

ZCWPW1

ZCWPW2

ZEB1

ZEB2

ZFAT

ZFHX2

ZFHX3

ZFHX4

ZFP1

ZFP14

ZFP2

ZFP28

ZFP295

ZFP3

ZFP30

ZFP37

ZFP41

ZFP42

ZFP57

ZFP62

ZFP64

ZFP69

ZFP69B

ZFP740

ZFP787

ZFP82

ZFP90

ZFP91

ZFP92

ZFPM1

ZFPM2

ZFX

ZFY

ZGLP1

ZGPAT

ZHX1

ZHX2

ZHX3

ZIC1

ZIC2 
ZIC3

ZIC4

ZIC5

ZIK1

ZIM2

ZIM3

ZKSCAN1

ZKSCAN2

ZKSCAN3

ZKSCAN4

ZKSCAN5

ZKSCAN7

ZKSCAN8

ZMAT1

ZMAT4

ZMYM2

ZMYM3

ZMYM4

ZMYND11

ZMYND8

ZNF10

ZNF100

ZNF101

ZNF107

ZNF112

ZNF114

ZNF117

ZNF12

ZNF121

ZNF124

ZNF131

ZNF132

ZNF133

ZNF134

ZNF135

ZNF136

ZNF138

ZNF14

ZNF140

ZNF141

ZNF142

ZNF143

ZNF146

ZNF148

ZNF154

ZNF155

ZNF157

ZNF16

ZNF160

ZNF165

ZNF169 
ZNF17

ZNF174

ZNF175

ZNF177

ZNF18

ZNF180

ZNF181

ZNF182

ZNF184

ZNF189

ZNF19

ZNF195

ZNF197

ZNF2

ZNF20

ZNF200

ZNF202

ZNF205

ZNF207

ZNF208

ZNF211

ZNF212

ZNF213

ZNF214

ZNF215

ZNF217

ZNF219

ZNF22

ZNF221

ZNF222

ZNF223

ZNF224

ZNF225

ZNF226

ZNF227

ZNF229

ZNF23

ZNF230

ZNF232

ZNF233

ZNF234

ZNF235

ZNF236

ZNF239

ZNF24

ZNF248

ZNF25

ZNF250

ZNF251

ZNF253

ZNF254 
ZNF256

ZNF257

ZNF26

ZNF260

ZNF263

ZNF264

ZNF266

ZNF267

ZNF268

ZNF273

ZNF274

ZNF275

ZNF276

ZNF277

ZNF28

ZNF280A

ZNF280B

ZNF280C

ZNF280D

ZNF281

ZNF282

ZNF283

ZNF284

ZNF285

ZNF286A

ZNF286B

ZNF287

ZNF292

ZNF296

ZNF3

ZNF30

ZNF300

ZNF302

ZNF304

ZNF311

ZNF316

ZNF317

ZNF318

ZNF319

ZNF32

ZNF320

ZNF322

ZNF324

ZNF324B

ZNF326

ZNF329

ZNF331

ZNF333

ZNF334

ZNF335

ZNF337 
ZNF33A

ZNF33B

ZNF34

ZNF341

ZNF343

ZNF345

ZNF346

ZNF347

ZNF35

ZNF350

ZNF354A

ZNF354B

ZNF354C

ZNF358

ZNF362

ZNF365

ZNF366

ZNF367

ZNF37A

ZNF382

ZNF383

ZNF384

ZNF385A

ZNF385B

ZNF385C

ZNF385D

ZNF391

ZNF394

ZNF395

ZNF396

ZNF397

ZNF398

ZNF404

ZNF407

ZNF408

ZNF41

ZNF410

ZNF414

ZNF415

ZNF416

ZNF417

ZNF418

ZNF419

ZNF420

ZNF423

ZNF425

ZNF426

ZNF428

ZNF429

ZNF43

ZNF430 
ZNF431

ZNF432

ZNF433

ZNF436

ZNF438

ZNF439

ZNF44

ZNF440

ZNF441

ZNF442

ZNF443

ZNF444

ZNF445

ZNF446

ZNF449

ZNF45

ZNF451

ZNF454

ZNF460

ZNF461

ZNF462

ZNF467

ZNF468

ZNF469

ZNF470

ZNF471

ZNF473

ZNF474

ZNF479

ZNF48

ZNF480

ZNF483

ZNF484

ZNF485

ZNF486

ZNF487

ZNF488

ZNF490

ZNF491

ZNF492

ZNF493

ZNF496

ZNF497

ZNF500

ZNF501

ZNF502

ZNF503

ZNF506

ZNF507

ZNF510

ZNF511 
ZNF512

ZNF512B

ZNF513

ZNF514

ZNF516

ZNF517

ZNF518A

ZNF518B

ZNF519

ZNF521

ZNF524

ZNF525

ZNF526

ZNF527

ZNF528

ZNF529

ZNF530

ZNF532

ZNF534

ZNF536

ZNF540

ZNF541

ZNF543

ZNF544

ZNF546

ZNF547

ZNF548

ZNF549

ZNF550

ZNF551

ZNF552

ZNF554

ZNF555

ZNF556

ZNF557

ZNF558

ZNF559

ZNF560

ZNF561

ZNF562

ZNF563

ZNF564

ZNF565

ZNF566

ZNF567

ZNF568

ZNF569

ZNF57

ZNF570

ZNF571

ZNF572 
ZNF573

ZNF574

ZNF575

ZNF576

ZNF577

ZNF578

ZNF579

ZNF580

ZNF581

ZNF582

ZNF583

ZNF584

ZNF585A

ZNF585B

ZNF586

ZNF587

ZNF587B

ZNF589

ZNF592

ZNF594

ZNF595

ZNF596

ZNF597

ZNF598

ZNF599

ZNF600

ZNF605

ZNF606

ZNF607

ZNF608

ZNF609

ZNF610

ZNF611

ZNF613

ZNF614

ZNF615

ZNF616

ZNF618

ZNF619

ZNF620

ZNF621

ZNF623

ZNF624

ZNF625

ZNF626

ZNF627

ZNF628

ZNF629

ZNF630

ZNF638

ZNF639 
ZNF641

ZNF644

ZNF645

ZNF646

ZNF648

ZNF649

ZNF652

ZNF653

ZNF654

ZNF655

ZNF658

ZNF66

ZNF660

ZNF662

ZNF664

ZNF665

ZNF667

ZNF668

ZNF669

ZNF670

ZNF671

ZNF672

ZNF674

ZNF675

ZNF676

ZNF677

ZNF678

ZNF679

ZNF680

ZNF681

ZNF682

ZNF683

ZNF684

ZNF687

ZNF688

ZNF689

ZNF69

ZNF691

ZNF692

ZNF695

ZNF696

ZNF697

ZNF699

ZNF7

ZNF70

ZNF700

ZNF701

ZNF703

ZNF704

ZNF705A

ZNF705B 
ZNF705D

ZNF705E

ZNF705G

ZNF706

ZNF707

ZNF708

ZNF709

ZNF71

ZNF710

ZNF711

ZNF713

ZNF714

ZNF716

ZNF717

ZNF718

ZNF721

ZNF724

ZNF726

ZNF727

ZNF728

ZNF729

ZNF730

ZNF732

ZNF735

ZNF736

ZNF737

ZNF74

ZNF740

ZNF746

ZNF747

ZNF749

ZNF750

ZNF75A

ZNF75D

ZNF76

ZNF761

ZNF763

ZNF764

ZNF765

ZNF766

ZNF768

ZNF77

ZNF770

ZNF771

ZNF772

ZNF773

ZNF774

ZNF775

ZNF776

ZNF777

ZNF778 
ZNF780A

ZNF780B

ZNF781

ZNF782

ZNF783

ZNF784

ZNF785

ZNF786

ZNF787

ZNF788

ZNF789

ZNF79

ZNF790

ZNF791

ZNF792

ZNF793

ZNF799

ZNF8

ZNF80

ZNF800

ZNF804A

ZNF804B

ZNF805

ZNF808

ZNF81

ZNF813

ZNF814

ZNF816

ZNF821

ZNF823

ZNF827

ZNF829

ZNF83

ZNF830

ZNF831

ZNF835

ZNF836

ZNF837

ZNF84

ZNF841

ZNF843

ZNF844

ZNF845

ZNF846

ZNF85

ZNF850

ZNF852

ZNF853

ZNF860

ZNF865

ZNF878 
ZNF879

ZNF880

ZNF883

ZNF888

ZNF891

ZNF90

ZNF91

ZNF92

ZNF93

ZNF98

ZNF99

ZNFX1

ZNHIT1

ZSCAN1

ZSCAN10

ZSCAN12

ZSCAN16

ZSCAN18

ZSCAN2

ZSCAN20

ZSCAN21

ZSCAN22

ZSCAN23

ZSCAN25

ZSCAN26

ZSCAN29

ZSCAN30

ZSCAN31

ZSCAN32

ZSCAN4

ZSCAN5A

ZSCAN5B

ZSCAN5C

ZSCAN9

ZUFSP

ZXDA

ZXDB

ZXDC

ZZZ3

BCL7A

BCL7B

BCL7C 


\begin{tabular}{|c|c|c|c|c|c|}
\hline $\begin{array}{l}460 \text { cancer driver genes } \\
\text { (Dietlin et al 2020) }\end{array}$ & $\begin{array}{l}299 \text { cancer driver } \\
\text { genes (Bailey et al } \\
\text { 2018) }\end{array}$ & $\begin{array}{l}369 \text { cancer driver } \\
\text { genes } \\
\text { (Martincorena et } \\
\text { al. 2017) }\end{array}$ & $\begin{array}{l}260 \text { cancer driver } \\
\text { genes (Lawrence et } \\
\text { al. 2014) }\end{array}$ & $\begin{array}{l}\text { Loss of function mutations } \\
\text { intolerant genes (Lek et al. } \\
\text { 2016) }\end{array}$ & $\begin{array}{l}127 \text { most commonly } \\
\text { mutated genes in Cancer } \\
\text { (Kandoth 2013) }\end{array}$ \\
\hline TP53 & ABL1 & ABL1 & ACO1 & SKI & MIR142 \\
\hline KRAS & ACVR1 & ACO1 & ACVR1B & GNB1 & B4GALT3 \\
\hline TP53 & ACVR1B & ACVR1 & ACVR2B & GABRD & EGR3 \\
\hline APC & ACVR2A & ACVR1B & ADNP & RER1 & CRIPAK \\
\hline TP53 & AJUBA & ACVR2A & AJUBA & PRDM16 & PRX \\
\hline TP53 & AKT1 & ACVR2B & AKT1 & TP73 & LIFR \\
\hline BRAF & ALB & ADNP & ALK & PANK4 & $A R$ \\
\hline PTEN & ALK & AJUBA & ALKBH6 & ICMT & EPPK1 \\
\hline TP53 & AMER1 & AKT1 & ALPK2 & AJAP1 & HGF \\
\hline TP53 & APC & ALB & ANK3 & CHD5 & NPM1 \\
\hline PIK3CA & APOB & ALK & $\mathrm{APC}$ & PHF13 & USP9X \\
\hline VHL & $A R$ & ALPK2 & APOL2 & DNAJC11 & NCOR1 \\
\hline TP53 & ARAF & AMER1 & ARHGAP35 & PIK3CD & POLQ \\
\hline BRAF & ARHGAP35 & APC & ARID1A & CAMTA1 & ARHGAP35 \\
\hline GNA11 & ARID1A & APOL2 & ARID2 & PARK7 & MALAT1 \\
\hline TP53 & ARID2 & ARHGAP35 & ARID5B & RERE & LRRK2 \\
\hline KRAS & ARID5B & ARHGAP5 & ASXL1 & TARDBP & NOTCH1 \\
\hline TP53 & ASXL1 & ARID1A & ASXL2 & PGD & NAV3 \\
\hline IDH1 & ASXL2 & ARID1B & ATM & CASZ1 & STK11 \\
\hline TP53 & ATF7IP & ARID2 & ATP5B & KIF1B & MTOR \\
\hline GTF2I & ATM & ARID5B & AXIN2 & UBE4B & RPL5 \\
\hline TP53 & ATR & ASXL1 & AZGP1 & MFN2 & RPL22 \\
\hline TP53 & ATRX & ATM & B2M & MTOR & PTPN11 \\
\hline PIK3CA & ATXN3 & ATP1A1 & BAP1 & VPS13D & PPP2R1A \\
\hline ARID1A & AXIN1 & ATP1B1 & BCLAF1 & PRDM2 & NFE2L3 \\
\hline PBRM1 & AXIN2 & ATP2B3 & $\mathrm{BCOR}$ & SPEN & NFE2L2 \\
\hline KRAS & $\mathrm{B} 2 \mathrm{M}$ & ATRX & BHMT2 & ZBTB17 & $\mathrm{IDH} 2$ \\
\hline PIK3R1 & BAP1 & AXIN1 & BRAF & DDI2 & IDH1 \\
\hline CTNNB1 & $\mathrm{BCL2}$ & AXIN2 & BRCA1 & $\mathrm{FBXO42}$ & TET2 \\
\hline BAP1 & BCL2L11 & AZGP1 & BRE & UBR4 & DNMT3A \\
\hline PIK3CA & $\mathrm{BCOR}$ & B2M & C3orf70 & EIF4G3 & AJUBA \\
\hline TP53 & BRAF & BAP1 & CAP2 & ECE1 & $\mathrm{CDH} 1$ \\
\hline NRAS & BRCA1 & BCLAF1 & CARD11 & HP1BP3 & PCBP1 \\
\hline ATRX & BRCA2 & $\mathrm{BCOR}$ & CASP8 & RAP1GAP & U2AF1 \\
\hline CTNNB1 & BRD7 & BHMT2 & CBFB & EPHB2 & SF3B1 \\
\hline BAP1 & BTG2 & BIRC3 & CCDC120 & USP48 & SPOP \\
\hline KDM6A & CACNA1A & BMPR2 & CCDC6 & KDM1A & KEAP1 \\
\hline SF3B1 & CARD11 & BRAF & CCND1 & HNRNPR & FBXW7 \\
\hline PIK3CA & CASP8 & BRCA1 & CD1D & TCEB3 & HIST1H2BD \\
\hline ARID1A & CBFB & BRCA2 & CD70 & LYPLA2 & $\mathrm{H} 3 \mathrm{~F} 3 \mathrm{C}$ \\
\hline TP53 & CBWD3 & BRD7 & CD79B & $\mathrm{E} 2 \mathrm{~F} 2$ & HIST1H1C \\
\hline NF2 & CCND1 & C3orf70 & $\mathrm{CDC} 27$ & TMEM57 & sox17 \\
\hline FBXW7 & CD70 & CACNA1D & $\mathrm{CDH} 1$ & GRHL3 & TBL1XR1 \\
\hline KRAS & CD79B & CALR & CDK12 & SRRM1 & AXIN2 \\
\hline FAT1 & $\mathrm{CDH} 1$ & CARD11 & CDK4 & WDTC1 & CTNNB1 \\
\hline SMAD4 & CDK12 & CASP8 & CDKN1A & ARID1A & $\mathrm{APC}$ \\
\hline КМт2C & CDK4 & CBFB & CDKN1B & SLC9A1 & ACVR2A \\
\hline TP53 & CDKN1A & $\mathrm{CBL}$ & CDKN2A & EYA3 & SMAD2 \\
\hline ARID1A & CDKN1B & CBLB & CEBPA & PPP1R8 & ACVR1B \\
\hline PIK3CA & CDKN2A & CCDC120 & CEP76 & AHDC1 & TGFBR2 \\
\hline NOTCH1 & CDKN2C & CCDC6 & CHD4 & WASF2 & SMAD4 \\
\hline CDKN2A & CEBPA & CCND1 & CHD8 & SRSF4 & AKT1 \\
\hline NF1 & $\mathrm{CHD} 3$ & CD1D & CNBD1 & PUM1 & PIK3CG \\
\hline FLG & CHD4 & CD58 & CNKSR1 & PTPRU & TLR4 \\
\hline RB1 & CHD8 & CD70 & COL5A1 & GMEB1 & PIK3R1 \\
\hline KMT2D & CHEK2 & CD79A & COL5A3 & YTHDF2 & PTEN \\
\hline PIM1 & $\mathrm{CIC}$ & CD79B & CREBBP & BAI2 & PIK3CA \\
\hline KMT2D & CNBD1 & $\mathrm{CDC} 27$ & CTCF & KPNA6 & MAPK8IP1 \\
\hline PIK3CA & COL5A1 & $\mathrm{CDC73}$ & CTNNB1 & TXLNA & MAP2K4 \\
\hline CTCF & CREB3L3 & $\mathrm{CDH} 1$ & CUL4B & KHDRBS1 & NRAS \\
\hline EGFR & CREBBP & $\mathrm{CDH} 10$ & CUX1 & ZBTB8OS & BRAF \\
\hline STK11 & CSDE1 & CDK12 & DDX3X & HDAC1 & MAP3К1 \\
\hline KEAP1 & CTCF & CDK4 & DDX5 & RBBP4 & NF1 \\
\hline
\end{tabular}




\begin{tabular}{|c|c|c|c|c|c|}
\hline CARD11 & CTNNB1 & CDKN1A & DIAPH1 & LCK & KRAS \\
\hline TP53 & CTNND1 & CDKN1B & DIS3 & S100PBP & CDKN2C \\
\hline ATRX & CUL1 & CDKN2A & DNAH12 & SFPQ & CDKN1A \\
\hline TP53 & CUL3 & CDKN2C & DNER & DLGAP3 & CCND1 \\
\hline ERCC2 & CYLD & CEBPA & DNMT3A & ZNF362 & CDKN1B \\
\hline PTEN & CYSLTR2 & CHD4 & EGFR & ZMYM4 & CDK12 \\
\hline PPP2R1A & DACH1 & CHD8 & EIF2S2 & AGO3 & RB1 \\
\hline TP53 & DAZAP1 & ClB3 & ELF3 & AGO4 & CDKN2A \\
\hline ARID2 & DDX3X & $\mathrm{CIC}$ & EP300 & MAP7D1 & FGFR3 \\
\hline FGFR3 & DHX9 & CMTR2 & EPHA2 & CLSPN & KIT \\
\hline EGFR & DIAPH2 & CNBD1 & ERBB2 & PSMB2 & FGFR2 \\
\hline PIK3CA & DICER1 & CNOT3 & ERBB3 & AGO1 & EPHB6 \\
\hline CDKN2A & DMD & COL2A1 & ERCC2 & NCDN & PDGFRA \\
\hline KIT & DNMT3A & COL5A1 & EZH1 & THRAP3 & ERBB4 \\
\hline KRAS & EEF1A1 & COL5A3 & EZH2 & STK40 & EPHA3 \\
\hline POLE & EEF2 & CREBBP & EZR & ZC $3 \mathrm{H} 12 \mathrm{~A}$ & FLT3 \\
\hline BCL2 & EGFR & CRLF2 & FAM166A & MTF1 & EGFR \\
\hline MYD88 & EGR3 & CSDE1 & FAM46C & SF3A3 & ERCC2 \\
\hline CREBBP & EIF1AX & CSF1R & FAT1 & SNIP1 & RAD21 \\
\hline CDKN2A & ELF3 & CSF3R & FBXW7 & GRIK3 & CHEK2 \\
\hline IGLL5 & EP300 & CTCF & FGFBP1 & PPIE & SMC3 \\
\hline STAG2 & EPAS1 & CTNNA1 & FGFR2 & PABPC4 & SMC1A \\
\hline CDKN1A & EPHA2 & CTNNB1 & FGFR3 & MACF1 & BRCA1 \\
\hline FGFR2 & EPHA3 & CUL3 & FLG & RLF & BAP1 \\
\hline RPL22 & ERBB2 & CUL4B & FLT3 & CTPS1 & STAG2 \\
\hline SETD2 & ERBB3 & CUX1 & FOXA1 & KCNQ4 & ATR \\
\hline TP53 & ERBB4 & CYLD & FOXQ1 & MFSD2A & BRCA2 \\
\hline HRAS & ERCC2 & DAXX & FRMD7 & FOXJ3 & ATRX \\
\hline CDH1 & ESR1 & DDX3X & GATA3 & SLC2A1 & ATM \\
\hline BRAF & $\mathrm{EZH} 2$ & DDX5 & GNA13 & YBX1 & TP53 \\
\hline RB1 & FAM46D & DIAPH1 & GNB1 & PTPRF & $\mathrm{EZH} 2$ \\
\hline ZNF804B & FAT1 & DICER1 & GNPTAB & ATP6VOB & ASXL1 \\
\hline ERBB4 & FBXW7 & DIS3 & GOT1 & KDM4A & ARID5B \\
\hline PIK3CA & FGFR1 & DNM2 & GPS2 & IPO13 & MLL4 \\
\hline GATA3 & FGFR2 & DNMT3A & GUSB & RNF220 & KDM6A \\
\hline IDH1 & FGFR3 & EEF1A1 & HIST1H1E & RPS8 & KDM5C \\
\hline NRAS & FLNA & EGFR & HIST1H3B & LRRC41 & SETBP1 \\
\hline CASP8 & FLT3 & EIF1AX & HIST1H4E & DMBX1 & NSD1 \\
\hline BAP1 & FOXA1 & EIF2S2 & HLA-A & FAF1 & SETD2 \\
\hline BRAF & FOXA2 & ELF3 & HLA-B & ZFYVE9 & PBRM1 \\
\hline SPOP & FOXQ1 & EML4 & HRAS & OSBPL9 & ARID1A \\
\hline PTEN & FUBP1 & EP300 & HSP90AB1 & ELAVL4 & MLL2 \\
\hline NRAS & GABRA6 & EPAS1 & IDH1 & ZCCHC11 & MLL3 \\
\hline ALB & GATA3 & EPHA2 & IDH2 & NDC1 & FOXA2 \\
\hline HRAS & GNA11 & EPS8 & IL7R & ZYG11B & CEBPA \\
\hline NF1 & GNA13 & ERBB2 & ING1 & LRP8 & VEZF1 \\
\hline $\mathrm{ClC}$ & GNAQ & ERBB3 & INPPL1 & PRPF38A & ELF3 \\
\hline KMT2C & GNAS & ERCC2 & INTS12 & SSBP3 & sox9 \\
\hline CDKN2A & GPS2 & ERG & IPO7 & DHCR24 & CBFB \\
\hline RNF43 & GRIN2D & ESR1 & IRF4 & PPAP2B & PHF6 \\
\hline ARID1A & GTF2I & ETNK1 & IRF6 & DAB1 & FOXA1 \\
\hline RXRA & H3F3A & EZH2 & ITGB7 & USP24 & EIF4A2 \\
\hline CENPE & $\mathrm{H} 3 \mathrm{~F} 3 \mathrm{C}$ & FAM104A & ITPKB & NFIA & WT1 \\
\hline KDM6A & $\mathrm{HGF}$ & FAM166A & KDM5C & ROR1 & SIN3A \\
\hline CREBBP & HIST1H1C & FAM46C & KDM6A & USP1 & TBX3 \\
\hline TP53 & HIST1H1E & FAT1 & KEAP1 & JAK1 & MECOM \\
\hline SF3B1 & HLA-A & FBXO11 & KEL & SGIP1 & RUNX1 \\
\hline МАРЗК1 & HLA-B & FBXW7 & KIT & MIER1 & TSHZ2 \\
\hline AXIN1 & HRAS & FGFR1 & KLHL8 & SERBP1 & TAF1 \\
\hline APC & HUWE1 & FGFR2 & KRAS & LEPR & CTCF \\
\hline ARID1A & IDH1 & FGFR3 & LCTL & DNAJC6 & EP300 \\
\hline FOXA1 & IDH2 & FLG & MAP2K1 & SRSF11 & TSHZ3 \\
\hline SMARCA2 & IL6ST & FLT3 & MAP2K4 & ZRANB2 & GATA3 \\
\hline REV3L & IL7R & FOSL2 & МАР3К1 & NEGR1 & VHL \\
\hline NOTCH1 & INPPL1 & FOXA1 & MAP4K3 & LRRC7 & \\
\hline KRAS & IRF2 & FOXA2 & MBD1 & ANKRD13C & \\
\hline DNMT3A & IRF6 & FOXL2 & MED12 & FUBP1 & \\
\hline
\end{tabular}




\begin{tabular}{|c|c|c|c|c|}
\hline NRAS & JAK1 & FOXQ1 & MED23 & ZZZ3 \\
\hline FLT3 & JAK2 & FRMD7 & MET & USP33 \\
\hline NOTCH1 & JAK3 & FUBP1 & MGA & LPHN2 \\
\hline NPM1 & KANSL1 & GAGE12J & MICALCL & PKN2 \\
\hline KRAS & KDM5C & GATA1 & MLL & ZNF326 \\
\hline HRAS & KDM6A & GATA2 & MLL2 & ZNF644 \\
\hline RB1 & KEAP1 & GATA3 & MLL3 & ABCD3 \\
\hline PIK3CA & KEL & GNA11 & MLL4 & ARHGAP29 \\
\hline RAC1 & KIF1A & GNA13 & MORC4 & RPL5 \\
\hline KIT & KIT & GNAQ & MPO & BCAR3 \\
\hline SF3B1 & KLF5 & GNAS & MTOR & MTF2 \\
\hline ATM & KMT2A & GNB1 & MUC17 & FNBP1L \\
\hline RB1 & КMT2B & GNPTAB & MXRA5 & HIAT1 \\
\hline MAML2 & КMT2C & GPS2 & MYB & LPPR4 \\
\hline IDH1 & KMT2D & GTF2I & MYCN & LPPR5 \\
\hline PBRM1 & KRAS & GUSB & MYD88 & PTBP2 \\
\hline EZH2 & KRT222 & $\mathrm{H} 3 \mathrm{~F} 3 \mathrm{~A}$ & MYOCD & PRPF38B \\
\hline FUBP1 & LATS1 & H3F3B & NBPF1 & COL11A1 \\
\hline U2AF1 & LATS2 & HIST1H1C & NCOR1 & WDR47 \\
\hline GNAS & LEMD2 & HIST1H1E & NF1 & SORT1 \\
\hline HRAS & LZTR1 & HIST1H2BD & NFE2L2 & PSMA5 \\
\hline MYD88 & MACF1 & HIST1H3B & NOTCH1 & CELSR2 \\
\hline IDH1 & MAP2K1 & HIST1H4E & NPM1 & SARS \\
\hline IDH2 & MAP2K4 & HLA-A & NRAS & SLC6A17 \\
\hline ERBB2 & MAP3K1 & HLA-B & NSD1 & CSF1 \\
\hline XPO1 & MAР3К4 & HLA-C & NTN4 & RBM15 \\
\hline TET2 & MAPK1 & HNF1A & NUP210L & AHCYL1 \\
\hline IDH2 & MAX & HOXB3 & ODAM & RAP1A \\
\hline SF3B1 & MECOM & HRAS & OMA1 & CEPT1 \\
\hline AKT1 & MED12 & IDH1 & OR4A16 & HIPK1 \\
\hline NEFH & MEN1 & IDH2 & OR52N1 & CAPZA1 \\
\hline PIK3CA & MET & IKBKB & OTUD7A & MOV10 \\
\hline PBRM1 & MGA & IKZF1 & PAPD5 & SYCP1 \\
\hline HRAS & MGMT & IL6ST & PBRM1 & NRAS \\
\hline PTEN & MLH1 & IL7R & PCBP1 & TRIM33 \\
\hline CD79B & $\mathrm{MSH} 2$ & ING1 & PDAP1 & CSDE1 \\
\hline ARID2 & MSH3 & INTS12 & PDCD2L & ATP1A1 \\
\hline КМт2C & MSH6 & IPO7 & PDSS2 & IGSF3 \\
\hline CDH1 & MTOR & IRF4 & PHF6 & MAN1A2 \\
\hline CTNNB1 & MUC6 & ITGB7 & PIK3CA & ANKRD34A \\
\hline RBM10 & MYC & ITPKB & PIK3R1 & RBM8A \\
\hline BRAF & MYCN & JAK1 & PLCG2 & NOTCH2 \\
\hline ERBB2 & MYD88 & JAK2 & POLE & PIAS3 \\
\hline NSD1 & MYH9 & JAK3 & POU2AF1 & FCGR1A \\
\hline MAP2K4 & NCOR1 & KANSL1 & POU2F2 & SF3B4 \\
\hline ARID1A & NF1 & KCNJ5 & PPM1D & ANP32E \\
\hline ZNF479 & NF2 & KDM5C & PPP2R1A & PRPF3 \\
\hline PTEN & NFE2L2 & KDM6A & PPP6C & PLEKHO1 \\
\hline RB1 & NIPBL & KDR & PRDM1 & RPRD2 \\
\hline CHEK2 & NOTCH1 & KEAP1 & PTEN & MCL1 \\
\hline U2AF1 & $\mathrm{NOTCH} 2$ & KEL & PTPN11 & ARNT \\
\hline TGFBR2 & NPM1 & KIT & QKI & SETDB1 \\
\hline BRAF & NRAS & KLF4 & RAB40A & CERS2 \\
\hline RPS6KA3 & NSD1 & KLF5 & RAC1 & PI4KB \\
\hline ERBB3 & NUP133 & KLHL8 & RAD21 & SNX27 \\
\hline KMT2D & NUP93 & KMT2A & RASA1 & POGZ \\
\hline PIK3R1 & PAX5 & КМТ2B & RB1 & PSMD4 \\
\hline CEBPA & PBRM1 & КМт2C & RBM10 & ZNF687 \\
\hline SETD2 & РCBP1 & KMT2D & RHEB & RORC \\
\hline HLA-A & PDGFRA & KRAS & RHOA & CHTOP \\
\hline $\mathrm{EZH} 2$ & PDS5B & KRT5 & RIT1 & CRTC2 \\
\hline PTEN & PGR & LATS2 & RPL5 & INTS3 \\
\hline APC & PHF6 & LCTL & RPS15 & ILF2 \\
\hline $\mathrm{BACH} 1$ & PIK3CA & LZTR1 & RPS2 & GATAD2B \\
\hline APC & PIK3CB & MAP2K1 & RSBN1L & DENND4B \\
\hline U2AF1 & PIK3CG & MAP2K2 & RUNX1 & UBAP2L \\
\hline BAP1 & PIK3R1 & MAP2K4 & RXRA & ADAR \\
\hline
\end{tabular}




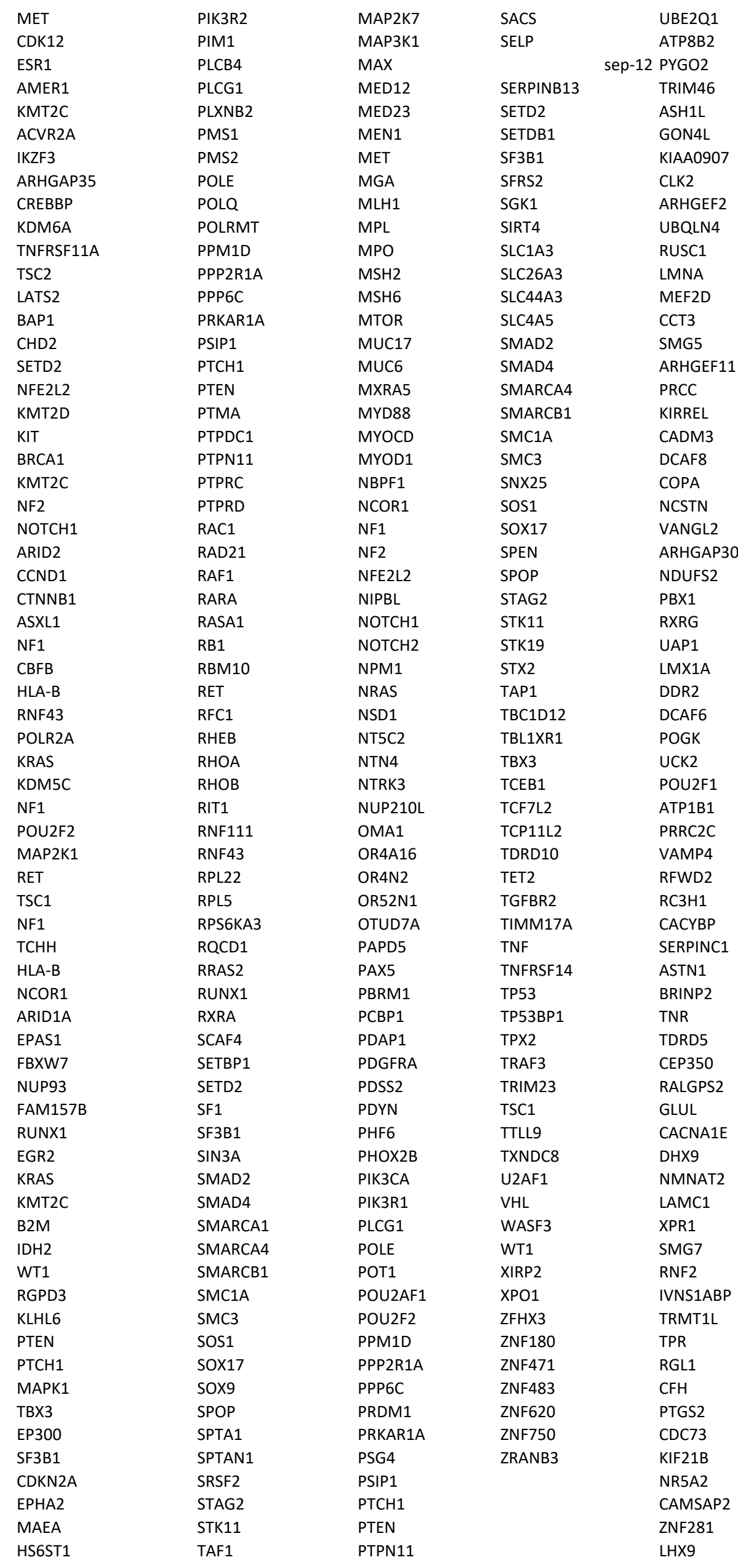




\begin{tabular}{|c|c|c|c|}
\hline ACPP & TBL1XR1 & PTPRB & PTPRC \\
\hline ARID1A & TBX3 & QKI & ELF3 \\
\hline BCOR & TCEB1 & RAC1 & IPO9 \\
\hline CS & TCF12 & RACGAP1 & NAV1 \\
\hline RHPN2 & TCF7L2 & RAD21 & TMEM183A \\
\hline ZNF280D & TET2 & RASA1 & PIK3C2B \\
\hline FBXW7 & TGFBR2 & RB1 & $\mathrm{ZC} 3 \mathrm{H} 11 \mathrm{~A}$ \\
\hline PIK3CA & TGIF1 & RBM10 & MDM4 \\
\hline SETD2 & THRAP3 & RET & PLEKHA6 \\
\hline RHOA & TLR4 & RHEB & RBBP5 \\
\hline CNBD1 & TMSB4X & RHOA & SOX13 \\
\hline NF1 & TNFAIP3 & RHOB & NFASC \\
\hline KRAS & TP53 & RIT1 & NUCKS1 \\
\hline SOX9 & TRAF3 & RNF43 & MAPKAPK2 \\
\hline BRD7 & TSC1 & RPL10 & SYT14 \\
\hline KRAS & $\mathrm{TSC} 2$ & RPL22 & IRF6 \\
\hline PPP6C & TXNIP & RPL5 & PLXNA2 \\
\hline ERBB2 & U2AF1 & RPS15 & SLC30A1 \\
\hline ELF3 & UNCX & RPS2 & PROX1 \\
\hline KMT2D & USP9X & RPS6KA3 & LPGAT1 \\
\hline GNAQ & VHL & RREB1 & PTPN14 \\
\hline BIRC3 & WHSC1 & RUNX1 & TGFB2 \\
\hline PTEN & WT1 & RXRA & MARK1 \\
\hline AKT1 & XPO1 & SELP & ESRRG \\
\hline ELF3 & ZBTB20 & SETBP1 & KCTD3 \\
\hline NFE2L2 & ZBTB7B & SETD2 & RAB3GAP2 \\
\hline NFE2L2 & $\mathrm{ZC} 3 \mathrm{H} 12 \mathrm{~A}$ & SF3B1 & SLC30A10 \\
\hline APC & ZCCHC12 & SGK1 & FBXO28 \\
\hline BRCA2 & ZFHX3 & $\mathrm{SH} 2 \mathrm{~B} 3$ & WDR26 \\
\hline IL6ST & ZFP36L1 & SLC1A3 & ENAH \\
\hline PBRM1 & ZFP36L2 & SLC26A3 & LBR \\
\hline SMAD4 & ZMYM2 & SLC44A3 & ACBD3 \\
\hline SMARCA4 & ZMYM3 & SLC4A5 & LIN9 \\
\hline ATM & ZNF133 & SMAD2 & ITPKB \\
\hline CLIP1 & ZNF750 & SMAD4 & CDC42BPA \\
\hline RUNX1 & & SMARCA4 & TAF5L \\
\hline RBM12 & & SMARCB1 & TSNAX \\
\hline HNRNPM & & SMC1A & EGLN1 \\
\hline EMG1 & & SMC3 & EXOC8 \\
\hline ERC1 & & SMO & SPRTN \\
\hline PIK3CA & & SMTNL2 & SIPA1L2 \\
\hline CDKN1B & & SNX25 & HEATR1 \\
\hline BRCA2 & & SOCS1 & RYR2 \\
\hline B2M & & sox17 & LYST \\
\hline TYRO3 & & SOX9 & ARID4B \\
\hline FBXW7 & & SPEN & ACTN2 \\
\hline MGA & & SPOP & CHRM3 \\
\hline STK19 & & SPTAN1 & FMN2 \\
\hline TMPRSS15 & & SRC & AKT3 \\
\hline TCF12 & & SRSF2 & ZBTB18 \\
\hline TP53 & & STAG2 & CNST \\
\hline CDKN2A & & STAT3 & DESI2 \\
\hline PTEN & & STAT5B & KIF26B \\
\hline CDC27 & & STK11 & AHCTF1 \\
\hline FOXA1 & & STK19 & ZNF496 \\
\hline KDM6A & & STX2 & HNRNPU \\
\hline GNA13 & & SUFU & MYT1L \\
\hline TMPRSS13 & & TBC1D12 & RPS7 \\
\hline CNOT9 & & TBL1XR1 & ODC1 \\
\hline AJUBA & & TBX3 & RRM2 \\
\hline BRAF & & TCEB1 & ASAP2 \\
\hline CHD4 & & TCF12 & RNF144A \\
\hline ACVR1B & & TCF7L2 & ADAM17 \\
\hline TPTE2 & & TCP11L2 & GRHL1 \\
\hline GPS2 & & TDRD10 & MYCN \\
\hline LARP4B & & TERT & DDX1 \\
\hline RB1 & & TET2 & TRIB2 \\
\hline
\end{tabular}




\begin{tabular}{|c|c|c|}
\hline CLIP1 & TG & ROCK2 \\
\hline TRAF3 & TGFBR2 & PUM2 \\
\hline ZNF430 & TGIF1 & NCOA1 \\
\hline XYLT2 & TIMM17A & ATAD2B \\
\hline CDKN1B & TNF & KIF3C \\
\hline SMAD4 & TNFAIP3 & DPYSL5 \\
\hline MGA & TNFRSF14 & ASXL2 \\
\hline NRAS & TOP2A & RAB10 \\
\hline EP300 & TP53 & ZNF513 \\
\hline BCOR & TRAF3 & CAD \\
\hline STAG2 & TRAF7 & GTF3C2 \\
\hline ZFHX3 & TRIM23 & NRBP1 \\
\hline CTNNB1 & TSC1 & ZNF512 \\
\hline MBD6 & TSC2 & FOSL2 \\
\hline NUMBL & TSHR & PPP1CB \\
\hline ARID1A & TTLL9 & PPM1G \\
\hline RB1 & TYRO3 & WDR43 \\
\hline TET2 & U2AF1 & SPAST \\
\hline TNFRSF14 & UBR5 & CRIM1 \\
\hline KMT2D & UPF3A & STRN \\
\hline IDH1 & VHL & BIRC6 \\
\hline SPEN & WASF3 & SRSF7 \\
\hline ZFP36L1 & WT1 & SLC8A1 \\
\hline PIK3CA & XIRP2 & ATL2 \\
\hline sox9 & XPO1 & SOS1 \\
\hline ZNF292 & ZBTB20 & TMEM178A \\
\hline FUBP1 & ZBTB7B & EPAS1 \\
\hline HMGCR & ZFHX3 & PRKCE \\
\hline PEG3 & ZFP36L1 & FBXO11 \\
\hline TSC1 & ZFP36L2 & PSME4 \\
\hline USP28 & ZFX & SPTBN1 \\
\hline HRAS & ZMYM3 & CCDC88A \\
\hline ERF & ZNF471 & NRXN1 \\
\hline ZC3H13 & ZNF620 & CCT4 \\
\hline KDM6A & ZNF750 & REL \\
\hline BTG2 & ZNF800 & PNPT1 \\
\hline RB1 & ZNRF3 & XPO1 \\
\hline NEFH & ZRSR2 & PAPOLG \\
\hline LRRC37A3 & & USP34 \\
\hline FAT1 & & EFEMP1 \\
\hline DLC1 & & MEIS1 \\
\hline CHIT1 & & VPS54 \\
\hline РОT1 & & PELI1 \\
\hline ZNF233 & & ETAA1 \\
\hline ARID1A & & EHBP1 \\
\hline CDKN2A & & ACTR2 \\
\hline PNPLA4 & & MDH1 \\
\hline ZFHX3 & & AFTPH \\
\hline $\mathrm{CDH} 1$ & & ANTXR1 \\
\hline ERBB3 & & GFPT1 \\
\hline TMEMЗОA & & AAK1 \\
\hline CIITA & & ADD2 \\
\hline PABPC1 & & FBXO41 \\
\hline MAP2K1 & & CCT7 \\
\hline ERBB2 & & ZNF638 \\
\hline P2RY8 & & CYP26B1 \\
\hline FOXA2 & & TET3 \\
\hline RHPN2 & & INO80B \\
\hline RHOA & & PCGF1 \\
\hline SETD2 & & CTNNA2 \\
\hline LILRA3 & & LRRTM1 \\
\hline MSH3 & & RMND5A \\
\hline MIA2 & & POLR1A \\
\hline PBRM1 & & KDM3A \\
\hline ERBB3 & & REEP1 \\
\hline EEF1A1 & & KANSL3 \\
\hline SGK1 & & SNRNP200 \\
\hline
\end{tabular}


MEF2B

UBXN11

HJURP

KMT2D

SF3B1

NCOA6

KMT2A

PTEN

KMT2D

FGFRL1

FAM104B

RB1

BAP1

KCNT2

ZMYM3

SOX17

FBXW7

ZNF14

FBXW7

ERBB3

FRMD4A

CTNND1

HLA-B

TP53

CDKN1A

NRAS

RBBP6

STAG2

MED12

DOT1L

EP300

RAD21

RBM10

HLA-DRB1

PAX5

HLA-A

RB1

ATM

PRSS48

ARID2

CCDC91

ZFHX3

MTOR

NUP93

HLA-A

ZNF814

HLA-A

ERICH6B

NUP210L

USP9X

UBE2A

SPEN

HLA-A

WNK1

ACVR2A

KDM6A

NF1

TNFAIP3

PBRM1

C3orf70

ZNF234

SPOP

CASP8

TENT5D

BCOR

PTEN

CTNNB1
ARID5A

STARD7

SEMA4C

INPP4A

TMEM131

AFF3

CNOT11

REV1

MAP4K4

NPAS2

RANBP2

BCL2L11

SH3RF3

POLR1B

ZC3H6

IL1B

TTL

ACTR3

CBWD2

RABL2A

DPP10

PAX8

MAP3K2

PTPN4

CLASP1

TFCP2L1

INHBB

TSN

GLI2

HS6ST1

WDR33

AMMECR1L

SAP130

IWS1

UBXN4

MCM6

MGAT5

ACVR2A

EPC2

KIF5C

ZEB2

RND3

LRP1B

MBD5

RIF1

NR4A2

ACVR1

FMNL2

KCNJ3

PRPF40A

MARCH7

TBR1

KCNH7

BAZ2B

RBMS1

TANK

SCN1A

SCN3A

STK39

SCN2A

PPIG

TLK1

LRP2

UBR3

RAPGEF4

DLX1

SP3 


\begin{tabular}{|c|c|}
\hline TP53BP1 & WIPF1 \\
\hline AKAP2 & HNRNPA3 \\
\hline PIK3R1 & SESTD1 \\
\hline NFE2L2 & OSBPL6 \\
\hline SSC5D & AGPS \\
\hline ZNF292 & CALCRL \\
\hline VCX & COL5A2 \\
\hline HNF1A & ZC3H15 \\
\hline ZNF750 & NCKAP1 \\
\hline ATM & COL3A1 \\
\hline ATM & STAT4 \\
\hline CTCF & SLC40A1 \\
\hline CTNNB1 & GLS \\
\hline MB21D2 & STAT1 \\
\hline CREBBP & SF3B1 \\
\hline FAT3 & HSPD1 \\
\hline PIK3R1 & SLC39A10 \\
\hline ARID1B & HECW2 \\
\hline ERRFI1 & ORC2 \\
\hline DTX1 & FAM126B \\
\hline HRNR & CFLAR \\
\hline STAT3 & SATB2 \\
\hline TRAK1 & SPATS2L \\
\hline BAZ2A & BMPR2 \\
\hline ZFP36L1 & ADAM23 \\
\hline SPEN & KLF7 \\
\hline CTNND2 & INO80D \\
\hline КМт2C & RAPH1 \\
\hline CDK12 & $A B I 2$ \\
\hline POLQ & CREB1 \\
\hline FGFR2 & MAP2 \\
\hline КМт2C & CCNYL1 \\
\hline NF1 & PIKFYVE \\
\hline ZFX & ERBB4 \\
\hline C10orf113 & XRCC5 \\
\hline AIM2 & IKZF2 \\
\hline NUDCD2 & ARPC2 \\
\hline ERBB4 & USP37 \\
\hline DDX3X & $\mathrm{RQCD} 1$ \\
\hline RBM10 & CTDSP1 \\
\hline RB1 & TNS1 \\
\hline PCCA & EPHA4 \\
\hline RASA1 & PAX3 \\
\hline RGS7 & SPEG \\
\hline TTK & FARSB \\
\hline KIF26B & NYAP2 \\
\hline AKR1D1 & CUL3 \\
\hline ZC3H13 & AGFG1 \\
\hline NF1 & TRIP12 \\
\hline HLA-B & PSMD1 \\
\hline RASA1 & EIF4E2 \\
\hline PIK3R1 & $\mathrm{NCL}$ \\
\hline NRAS & DGKD \\
\hline PTEN & GIGYF2 \\
\hline SETDB1 & NGEF \\
\hline CDK4 & ATG16L1 \\
\hline ZKSCAN4 & AGAP1 \\
\hline DHX30 & HDAC4 \\
\hline AKAP9 & ILKAP \\
\hline SMARCA4 & UBE2F \\
\hline КМT2C & PER2 \\
\hline NPFFR2 & HDLBP \\
\hline FAM86B2 & SNED1 \\
\hline ARID2 & KIF1A \\
\hline NCOR1 & ATG4B \\
\hline PABPC1 & GRM7 \\
\hline RRP36 & BHLHE40 \\
\hline
\end{tabular}




\begin{tabular}{|c|c|}
\hline JMJD1C & CNTN4 \\
\hline EYS & ITPR1 \\
\hline FGFR4 & SRGAP3 \\
\hline RIMS1 & BRPF1 \\
\hline SPATA31E1 & ARPC4 \\
\hline MAGI2 & SETD5 \\
\hline TBC1D5 & ATP2B2 \\
\hline DMWD & SLC6A1 \\
\hline NF1 & RAF1 \\
\hline AKR1C3 & IQSEC1 \\
\hline MAPK1 & FGD5 \\
\hline PPM1F & $\mathrm{NR} 2 \mathrm{C} 2$ \\
\hline NFKBIA & SLC6A6 \\
\hline ACVR2A & ANKRD28 \\
\hline TP53 & DAZL \\
\hline BAX & PLCL2 \\
\hline FRG1 & RARB \\
\hline MYB & UBE2E2 \\
\hline KANK3 & THRB \\
\hline MAP2K4 & КАТ2B \\
\hline IRF4 & TOP2B \\
\hline CHORDC1 & RPL15 \\
\hline ZBTB7A & TRIM71 \\
\hline SPHKAP & STT3B \\
\hline CDC27 & CNOT10 \\
\hline PRDM2 & DYNC1LI1 \\
\hline CLIP1 & CLASP2 \\
\hline PIK3CA & UBP1 \\
\hline SCN9A & SCN5A \\
\hline SF3B1 & WDR48 \\
\hline RB1 & OXSR1 \\
\hline ATP2B4 & ACVR2B \\
\hline ARID1A & CTNNB1 \\
\hline ATM & ZBTB47 \\
\hline MSH6 & RPL14 \\
\hline CCNQ & NKTR \\
\hline ZC3H12A & ZNF445 \\
\hline NUP153 & SNRK \\
\hline ARID2 & SETD2 \\
\hline PRSS50 & DHX30 \\
\hline RNF152 & CDC25A \\
\hline PSKH2 & SMARCC1 \\
\hline PTPN11 & KLHL18 \\
\hline ELF3 & MAP4 \\
\hline PCDH9 & CELSR3 \\
\hline BCL11B & IP6K2 \\
\hline $\mathrm{ClC}$ & QRICH1 \\
\hline C3orf70 & $\mathrm{ARIH} 2$ \\
\hline ARID5B & PRKAR2A \\
\hline SEMA4D & DAG1 \\
\hline DIS3 & RNF123 \\
\hline IGHMBP2 & USP19 \\
\hline GNA13 & BSN \\
\hline KDM6A & RBM5 \\
\hline HIST1H1C & CAMKV \\
\hline RPL21 & SEMA3F \\
\hline KEAP1 & RBM6 \\
\hline TPTE & GRM2 \\
\hline SPEN & TEX264 \\
\hline RHOB & RBM15B \\
\hline ATM & DOCK3 \\
\hline TEX13A & CACNA2D2 \\
\hline CUX1 & RAD54L2 \\
\hline HEPH & ALAS1 \\
\hline NCOR2 & WDR82 \\
\hline RASA1 & PBRM1 \\
\hline HOXC12 & WNT5A \\
\hline
\end{tabular}




\begin{tabular}{|c|c|}
\hline IRF8 & CACNA1D \\
\hline KLF3 & ARHGEF3 \\
\hline ARL16 & CACNA2D3 \\
\hline MAP2K7 & SFMBT1 \\
\hline PCNT & ERC2 \\
\hline ZNF479 & DCP1A \\
\hline GNA11 & FAM208A \\
\hline ANAPC1 & TMEM110 \\
\hline INMT & PRKCD \\
\hline $\mathrm{BCOR}$ & ARF4 \\
\hline ZIC4 & PXK \\
\hline LILRB4 & SLMAP \\
\hline DLGAP2 & MAGI1 \\
\hline RAD54B & PRICKLE2 \\
\hline ARID1A & THOC7 \\
\hline RARS2 & FEZF2 \\
\hline PRB4 & PSMD6 \\
\hline MGA & ATXN7 \\
\hline FAM8A1 & PDZRN3 \\
\hline FAT4 & MITF \\
\hline CDH11 & PPP4R2 \\
\hline RECQL4 & FOXP1 \\
\hline MARK2 & UBA3 \\
\hline PCDHA3 & EPHA6 \\
\hline ARID1A & $\mathrm{ROBO} 2$ \\
\hline TEAD3 & ZNF654 \\
\hline ESR1 & CADM2 \\
\hline ATM & TOMM70A \\
\hline MDN1 & NFKBIZ \\
\hline ARHGAP5 & SENP7 \\
\hline ATM & $\mathrm{BBX}$ \\
\hline ZNF750 & CBLB \\
\hline THSD7B & ALCAM \\
\hline CCND1 & ATG3 \\
\hline KDM6A & LSAMP \\
\hline DDX3X & TMEM39A \\
\hline PJA2 & ZBTB20 \\
\hline BOD1L1 & ARHGAP31 \\
\hline ARID1A & ATP6V1A \\
\hline RBM10 & KIAA2018 \\
\hline SHROOM3 & GSK3B \\
\hline ATM & STXBP5L \\
\hline ZNF804B & FSTL1 \\
\hline CCDC73 & GTF2E1 \\
\hline CHEK2 & CD86 \\
\hline YLPM1 & KPNA1 \\
\hline PRDM1 & PTPLB \\
\hline SF3B2 & ADCY5 \\
\hline NF1 & KALRN \\
\hline NCOR1 & ZNF148 \\
\hline $\mathrm{ZC} 3 \mathrm{H} 4$ & PODXL2 \\
\hline NOTCH1 & PLXNA1 \\
\hline CYLD & RUVBL1 \\
\hline STAT6 & SEC61A1 \\
\hline RELN & GATA2 \\
\hline ZNF208 & CNBP \\
\hline Sox9 & ISY1-RAB43 \\
\hline TGFBR2 & RAB7A \\
\hline PIK3CA & COPG1 \\
\hline MYB & ISY1 \\
\hline ZNF107 & PLXND1 \\
\hline RB1 & DNAJC13 \\
\hline $\mathrm{NOTCH} 2$ & ATP2C1 \\
\hline SELPLG & EPHB1 \\
\hline PKHD1L1 & TOPBP1 \\
\hline BDP1 & STAG1 \\
\hline BRD7 & PPP2R3A \\
\hline
\end{tabular}


AHNAK

PTCH1

BIRC6

CCDC66

ACVR2A

FLT3LG

RRAS2

ROCK2

SMTNL2

PTPN14

ARID2

KPNA4

PDCD10

$\mathrm{PHC3}$

SEC62

FNDC3B

TNIK

PIK3CA

TBL1XR1

ACTL6A

FXR1

DCUN1D1

IRF4

YEATS2

ATP11B

ATF7IP

EIF2B5

EPHB3

DVL3

PIXNA2

AP2M1

POLR2H

EIF4G1

PSMD2

TRA2B

IGF2BP2

EIF4A2

DNAJB11

SENP2

ETV5

MAP3K13

TP63

BCL6

ACAP2

ATP13A3

OPA1

PAK2

UBXN7

SENP5

RPL35A

DLG1

CTBP1

GAK

PCGF3

WHSC1

HTT

FAM193A

JAKMIP1

TBC1D14

KIAA0232

CRMP1

WDR1

LDB2 


\begin{tabular}{|c|c|}
\hline LTBP3 & BOD1L1 \\
\hline SON & FBXL5 \\
\hline NLRC5 & CPEB2 \\
\hline GPRIN2 & PPARGC1A \\
\hline EPS8 & KCNIP4 \\
\hline $\mathrm{NOTCH} 2$ & DHX15 \\
\hline SLIT1 & SLIT2 \\
\hline EVI5L & LCORL \\
\hline TPTE & GPR125 \\
\hline ARFGEF2 & RBPJ \\
\hline TMPRSS7 & RBM47 \\
\hline DBR1 & LIMCH1 \\
\hline CDK5RAP2 & PDS5A \\
\hline NCOR1 & UBE2K \\
\hline ZFHX4 & UCHL1 \\
\hline PBRM1 & RPL9 \\
\hline PFN1 & GABRB1 \\
\hline HLA-B & ATP8A1 \\
\hline ELF3 & BEND4 \\
\hline DYRK1A & GABRA4 \\
\hline TCF7L2 & GABRA2 \\
\hline RBM43 & FRYL \\
\hline IL6ST & USP46 \\
\hline PREX2 & CLOCK \\
\hline L3MBTL3 & PPAT \\
\hline CASZ1 & TMEM165 \\
\hline MED12 & $\mathrm{KIT}$ \\
\hline EOMES & KDR \\
\hline CR1 & PDGFRA \\
\hline SRGAP3 & YTHDC1 \\
\hline LILRB1 & LPHN3 \\
\hline ATP6V1B1 & REST \\
\hline SMARCB1 & POLR2B \\
\hline STK11 & UBA6 \\
\hline ARHGAP35 & SLC4A4 \\
\hline EPHA2 & RUFY3 \\
\hline FAM135B & ALB \\
\hline FRAS1 & ANKRD17 \\
\hline ITGA8 & USO1 \\
\hline $\mathrm{A} 1 \mathrm{CF}$ & NUP54 \\
\hline TPTE & G3BP2 \\
\hline TSC1 & CNOT6L \\
\hline NSD2 & COPS4 \\
\hline SCN9A & HNRNPDL \\
\hline ERBB2 & LIN54 \\
\hline ODF2L & WDFY3 \\
\hline ADGRV1 & HNRNPD \\
\hline STAG3L2 & PKD2 \\
\hline RECQL5 & BMPR1B \\
\hline FAT1 & GRID2 \\
\hline USH2A & SMARCAD1 \\
\hline FAT2 & TSPAN5 \\
\hline ICE1 & EIF4E \\
\hline MSH6 & DNAJB14 \\
\hline LAMA2 & РPР3СA \\
\hline PTCH1 & NFKB1 \\
\hline PHF6 & LEF1 \\
\hline U2AF1 & SEC24B \\
\hline ZC3H18 & ANK2 \\
\hline ADGRV1 & ELOVL6 \\
\hline PTPRC & CCNA2 \\
\hline ZNF750 & FAT4 \\
\hline MET & ANKRD50 \\
\hline PREX1 & ADAD1 \\
\hline UBR5 & ELF2 \\
\hline NOTCH1 & RAB33B \\
\hline EBF1 & NAA15 \\
\hline
\end{tabular}


FBXW7

FBXW7

NBEA

DCLK2

CD70

ZNF827

NR3C2

GNAS

EDNRA

PLRG1

KIAA0922

CFAP58

WBP1

RAPGEF2

ITGA6

STRIP2

GRIA2

NAF1

FNIP2

PDGFC

CPE

TLE1

KLHL2

CLCN3

HMGB2

MFAP3L

GPM6A

CDKN2AIP

TENM3

STOX2

TRIP13

SLC9A3

PAPD7

NSUN2

KIAA0947

ADCY2

SLC6A3

TRIO

CTNND2

CCT5

MARCH6

FAM105B

MTMR12

CDH6

PDZD2

CDH9

ZFR

DROSHA

SKP2

PRLR

NADK2

BRIX1

NUP155

DAB2

RICTOR

NIPBL

NNT

ZNF131

HMGCS1

PAIP1

HCN1

FST

MIER3

PLK2

GPBP1

IL6ST

DDX4

MAP3K1

KIF2A 


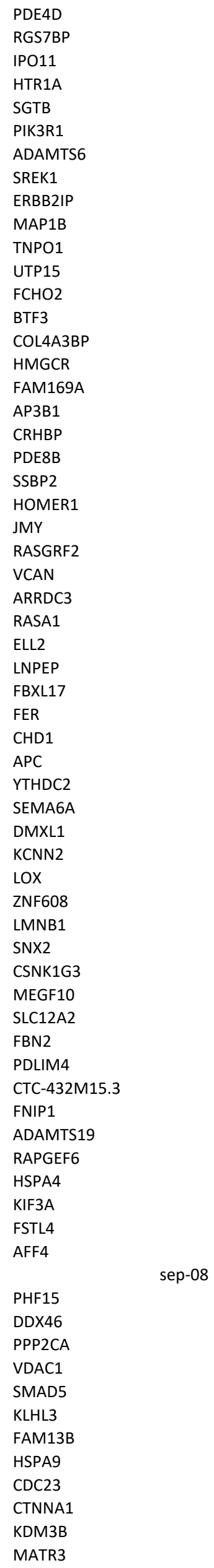


ETF1

LRRTM2

UBE2D2

NRG2

ANKHD1-EIF4EBP3

ANKHD1

ZMAT2

PCDHAC2

PCDHGC4

HDAC3

KCTD16

ARHGAP26

ARAP3

YIPF5

NDFIP1

RBM27

TCERG1

DPYSL3

JAKMIP2

FBXO38

PPP2R2B

CAMK2A

CSNK1A1

CSF1R

PPARGC1B

PDGFRB

SYNPO

NDST1

TNIP1

RBM22

DCTN4

G3BP1

LARP1

GRIA1

CYFIP2

SOX30

EBF1

TENM2

GABRA1

GABRG2

PWWP2A

GABRB2

DOCK2

LCP2

NPM1

SLIT3

FBXW11

CPEB4

CREBRF

UNC5A

FAF2

PDLIM7

FAM193B

DBN1

NSD1

ADAMTS2

HNRNPAB

FLT4

MAML1

HNRNPH1

TRIM41

GNB2L1

GMDS

IRF4

RREB1

PRPF4B

CDYL 
KIF13A

NUP153

FAM65B

C6orf62

GABBR1

TRIM39

PPP1R10

GNL1

DDX39B

BAG6

CSNK2B

ABHD16A

PRRC2A

AGPAT1

BRD2

COL11A2

RXRB

RING1

RPS18

SYNGAP1

PHF1

GRM4

SCUBE3

PPARD

PACSIN1

DEF6

RPS10

BRPF3

MAPK14

SRSF3

MTCH1

ZFAND3

MDGA1

RNF8

CMTR1

DAAM2

CCND3

TFEB

TRERF1

USP49

UBR2

GLTSCR1L

PPP2R5D

PTK7

KLHDC3

SRF

TTBK1

CUL9

SLC29A1

XPO5

HSP90AB1

TMEM63B

GPR116

RUNX2

CDC5L

OPN5

TFAP2D

ELOVL5

TFAP2B

GCM1

TRAM2

DST 
KCNQ5

SMAP1

PHF3

BAI3

SENP6

COL12A1

PHIP

EEF1A1

IBTK

SNAP91

DOPEY 1

TBX18

AKIRIN2

SYNCRIP

ZNF292

MAP3K7

EPHA7

MDN1

SIM1

CCNC

ATG5

GRIK2

PNISR

PRDM1

PREP

NR2E1

WASF1

REV3L

CDK19

CDC40

FYN

AMD1

HDAC2

HSF2

NCOA7

EPB41L2

PTPRK

L3MBTL3

SGK1

PDE7B

HBS1L

KIAA1244

MAP3K5

REPS1

MAP7

TNFAIP3

HIVEP2

GRM1

TAB2

STXBP5

LATS1

FBXO30

UST

ESR1

ZBTB2

FBXO5

SCAF8

ZDHHC14

ARID1B

TULP4

IGF2R

TAGAP

TCP1

EZR

WTAP

PDE10A

QKI 
MAP3K4

DLL1

MLLT4

GET4

CARD11

FOXK1

GNA12

EIF3B

TTYH3

CCZ1

USP42

ACTB

TNRC18

ETV1

THSD7A

BZW2

SNX13

SP4

HDAC9

AHR

ITGB8

RAPGEF5

MPP6

HNRNPA2B1

IGF2BP3

HOXA3

SKAP2

JAZF1

CREB5

HERPUD2

ANLN

KBTBD2

sep-07

RALA

PSMA2

ELMO1

GLI3

HECW1

$\mathrm{OGDH}$

ZMIZ2

ADCY1

TNS3

COBL

IKZF1

EGFR

CCT6A

AUTS2

STX1A

BAZ1B

LIMK1

NCF1

HIP1

EIF4H

GTF2IRD1

GTF2I

CLIP2

PTPN12

YWHAG

SRRM3

GNAI1

DBF4

SEMA3A

GRM3

DMTF1

HGF

CACNA2D1

PCLO 
ADAM22

ANKIB1

CDK6

CCDC132

COL1A2

LMTK2

TRRAP

SMURF1

GIGYF1

MEPCE

NYAP1

GNB2

ACTL6B

SRRT

EPHB4

ACHE

CUX1

NAMPT

SRPK2

PSMC2

RELN

DNAJC2

KMT2E

PRKAR2B

CBLL1

DOCK4

C7orf60

MET

CAPZA2

FOXP2

ST7

ING3

PTPRZ1

KCND2

CADPS2

WASL

ZNF800

SND1

FLNC

KLHDC10

UBE2H

AHCYL2

NRF1

MEST

MKLN1

PLXNA4

CALD1

SLC13A4

CNOT4

NUP205

TRIM24

JHDM1D

C7orf55-LUC7L2

HIPK2

KIAA1549

UBN2

DENND2A

BRAF

MKRN1

FAM131B

ZNF777

EZH2

ZNF398

CUL1

REPIN1

$\mathrm{KCNH} 2$

CDK5 
ABCF2

SLC4A2

AGAP3

RHEB

PRKAG2

KMT2C

ACTR3B

DPP6

RBM33

PAXIP1

$\mathrm{SHH}$

NCAPG2

DNAJB6

UBE3C

DLGAP2

XKR6

DLC1

LONRF1

CNOT7

DMTN

ATP6V1B2

XPO7

INTS10

CHMP7

CCAR2

DPYSL2

EBF2

PPP2R2A

HMBOX1

PTK2B

NRG1

MAK16

PURG

LSM1

ASH2L

WHSC1L1

FGFR1

ANK1

IKBKB

FNTA

PRKDC

VDAC3

KAT6A

HOOK3

ST18

RB1CC1

XKR4

LYN

CHD7

YTHDF3

RAB2A

PRDM14

MYBL1

ARFGEF1

VCPIP1

NCOA2

COPS5

KCNB2

STAU2

RDH10

UBE2W

RPL7

EYA1

HEY1

ZFHX4

ZNF704

STMN2 
MMP16

OSGIN2

WWP1

INTS8

RUNX1T1

KIAA1429

ESRP1

GDF6

GRHL2

PABPC1

UBR5

ANKRD46

ZFPM2

ANGPT1

LRP12

AZIN1

RIMS2

RAD21

CSMD3

TRPS1

EXT1

HAS2

SQLE

ATAD2

MTSS1

FBXO32

TRMT12

FAM49B

ASAP1

KCNQ3

PHF20L1

FAM135B

ARC

BAl1

PTK2

AGO2

ZC $3 \mathrm{H} 3$

SCRIB

CYC1

ADCK5

RPL8

CDC37L1

SMARCA2

RFX3

RCL1

UHRF2

JAK2

PTPRD

PSIP1

BNC2

NFIB

SH3GL2

MLLT3

KLHL9

TEK

ELAVL2

SMU1

NOL6

TOPORS

UBE2R2

UBAP1

CNTFR

UBAP2

RUSC2

TESK1

VCP

CD72 
TLN1

NPR2

PAX5

DCAF10

RNF38

CLTA

PGM5

RORB

ALDH1A1

GDA

ZFAND5

ABHD17B

SMC5

TLE4

TLE1

GNAQ

NAA35

UBQLN1

NTRK2

ZCCHC6

DAPK1

HNRNPK

SEMA4D

NFIL3

SYK

SPIN1

PHF2

IPPK

FAM120A

PTCH1

GABBR2

NCBP1

ANP32B

TGFBR1

ERP44

TEX10

SMC2

KLF4

ZNF462

SLC44A1

RAD23B

KIAA0368

EPB41L4B

UGCG

PRPF4

SNX30

PTBP3

ZNF618

COL27A1

PAPPA

RAB14

MEGF9

PHF19

DAB2IP

ASTN2

FBXW2

BRINP1

LHX6

$\mathrm{RC} 3 \mathrm{H} 2$

RABGAP1

LHX2

DENND1A

STRBP

PSMB7

SCAI

NR6A1

PPP6C 
NR5A1

RALGPS1

MAPKAP1

STXBP1

GAPVD1

$\mathrm{ClZ1}$

ENG

DNM1

SLC25A25

SPTAN1

ZER1

SET

GOLGA2

NUP188

PPP2R4

LRRC8A

NCS1

ABL1

PRRC2B

RAPGEF1

NUP214

TSC1

RPL7A

COL5A1

WDR5

RXRA

PPP1R26

OLFM1

NOTCH1

RABL6

ABCA2

TRAF2

FUT7

EDF1

GRIN1

ANAPC2

TUBB4B

CACNA1B

EHMT1

WDR37

GTPBP4

DIP2C

LARP4B

ZMYND11

KLF6

FAM208B

RBM17

TAF3

PRKCQ

SFMBT2

GDI2

SEPHS1

CAMK1D

UPF2

CELF2

SEC61A2

HSPA14

FAM171A1

SUV39H2

FRMD4A

VIM

MLLT10

STAM

MRC1

ARHGAP21

SVIL

MTPAP 
EPC1

CUL2

ITGB1

NRP1

RET

RASGEF1A

BMS1

MAPK8

PRKG1

CDK1

ANK3

UBE2D1

CCDC6

HERC4

JMJD1C

SIRT1

ARID5B

HNRNPH3

DDX21

TET1

H2AFY2

CCAR1

PSAP

NODAL

PPP3CB

MCU

KAT6B

$\mathrm{VCL}$

SEC24C

ZSWIM8

CAMK2G

ZMIZ1

DLG5

KCNMA1

GRID1

WAPAL

PTEN

HECTD2

PCGF5

SLC35G1

CPEB3

BTAF1

LGI1

KIF11

CCNJ

BLNK

HELLS

LCOR

C10orf12

PI4K2A

PIK3AP1

TM9SF3

SLIT1

CNNM1

C10orf2

CHUK

ERLIN1

PPRC1

FGF8

TRIM8

SUFU

ACTR1A 
NFKB2

CNNM2

PSD

WBP1L

XPNPEP1

TAF5

SORCS1

TCF7L2

ADD3

SMC3

SHOC2

TDRD1

FAM160B1

ABLIM1

ATRNL1

EMX2

RAB11FIP2

TIAL1

EIF3A

PDZD8

SLC18A2

GRK5

HSPA12A

MCMBP

FGFR2

ZRANB1

EBF3

INPP5A

PSMD13

PHRF1

MUC5B

AP2A2

CD81

NUP98

STIM1

NAP1L4

RRM1

ARFIP2

TRIM3

FAM160A2

APBB1

DCHS1

ST5

TMEM9B

DENND5A

USP47

CTR9

IPO7

WEE1

PSMA1

COPB1

ARNTL

SOX6

RPS13

GTF2H1

E2F8

TSG101

SPTY2D1

NAV2

KCNA4

LGR4

BDNF

PAX6

QSER1

CSTF3

$\mathrm{FBXO} 3$

MPPED2 
EIF3M

CAPRIN1

TRAF6

SLC1A2

EHF

LRRC4C

PRDM11

API5

TTC17

MAPK8IP1

DGKZ

CHRM4

CRY2

PHF21A

F2

AMBRA1

CKAP5

ATG13

CELF1

PSMC3

FNBP4

CLP1

SSRP1

SERPING1

ZDHHC5

RTN4RL2

ZFP91

CTNND1

OSBP

PATL1

DDB1

PRPF19

CPSF7

MYRF

SYT7

FADS2

DAGLA

FTH1

INCENP

HNRNPUL2

GANAB

MTA2

EEF1G

INTS5

SLC3A2

NXF1

CHRM1

STIP1

OTUB1

MARK2

NRXN2

PLCB3

RASGRP2

RPS6KA4

MEN1

SF1

PPP2R5B

ATG2A

SYVN1

DPF2

LTBP3

RELA

SF3B2

SART1

PACS1

KLC2

LRFN4 
ANKRD13D

RBM4

RBM14

KDM2A

RBM14-RBM4

SPTBN2

ADRBK1

PPP1CA

LRP5

SUV42OH1

CPT1A

PPP6R3

SHANK2

ANO1

PPFIA1

PDE2A

RNF121

NUMA1

FCHSD2

ARHGEF17

FAM168A

PPME1

POLD3

C11orf30

PRKRIR

RPS3

ARRB1

RSF1

EED

PCF11

RAB30

PICALM

GAB2

TENM4

FZD4

GRM5

FAT3

CWC15

MAML2

YAP1

SESN3

DCUN1D5

CUL5

GRIA4

SIK2

ZC3H12C

RDX

C11orf57

ZBTB16

CADM1

PAFAH1B2

SIK3

BACE1

DSCAML1

IL10RA

ARCN1

DDX6

KMT2A

BCL9L

IFT46

UBE4A

HYOU1

HMBS

HINFP

C2CD2L

GRAMD1B

HSPA8 
ARHGEF12

UBASH3B

TBCEL

SRPR

PKNOX2

STT3A

El24

PRDM10

ARHGAP32

KIRREL3

ZBTB44

VPS26B

IGSF9B

IQSEC3

WNK1

CACNA1C

FKBP4

KDM5A

CCND2

KCNA6

PRMT8

TNFRSF1A

CHD4

USP5

ZNF384

MLF2

ATN1

PTPN6

COPS7A

CDCA3

SLC2A14

CLSTN3

LPCAT3

FOXJ2

NANOG

PHC1

RIMKLB

LRP6

ETV6

ATF7IP

GRIN2B

WBP11

PLEKHA5

EPS8

AEBP2

SOX5

STK38L

ASUN

PTHLH

FAM60A

DENND5B

FAR2

IPO8

SLC2A13

CNTN1

GXYLT1

PRICKLE1

SLC38A2

SLC38A1

ARID2

SCAF11

NELL2

HDAC7

SLC38A4

CCNT1

SENP1

COL2A1 
SPATS2

SMARCD1

ASIC1

$\mathrm{KCNH} 3$

POU6F1

LARP4

CSRNP2

DIP2B

SLC4A8

ACVR1B

KRT6A

NR4A1

SCN8A

GRASP

KRT1

EIF4B

SPRYD3

RARG

ATF7

MAP3K12

SP1

ESPL1

PCBP2

ITGA5

NCKAP1L

HNRNPA1

HOXC6

PDE1B

SARNP

DGKA

CDK2

IKZF4

DNAJC14

GDF11

NABP2

PA2G4

SMARCC2

CS

PAN2

ANKRD52

ATP5B

BAZ2A

NAB2

KIF5A

DCTN2

LRP1

DTX3

MBD6

R3HDM2

CDK4

AGAP2

LEMD3

XPOT

MON2

USP15

TBK1

SRGAP1

DYRK2

GRIP1

RAP1B

MDM2

CPSF6 
CAND1

CCT2

FRS2

PTPRB

ZFC3H1

CNOT2

ZDHHC17

PPP1R12A

NAP1L1

OSBPL8

NAV3

PPFIA2

DUSP6

ATP2B1

PLXNC1

NR2C1

FGD6

NTN4

METAP2

UHRF1BP1L

ANKS1B

SCYL2

CDK17

HSP90B1

HCFC2

RFX4

SLC41A2

CORO1C

SART3

RIC8B

BTBD11

SSH1

ATP2A2

ANAPC7

ARPC3

GIT2

CUX2

ATXN2

VPS29

PPP1CC

NAA25

HECTD4

RPL6

PTPN11

MED13L

NOS1

FBXO21

TAOK3

TBX5

KSR2

TBX3

RPLPO

RAB35

MSI1

CIT

GCN1L1

CABP1

KDM2B

HNF1A

SPPL3

CLIP1

MLXIP

RSRC2

PITPNM2

SBNO1

SETD8

TMEM132D 
NCOR2

DHX37

RAN

ULK1

EP400

SFSWAP

GOLGA3

ZNF605

PSPC1

XPO4

ZMYM2

MPHOSPH8

RNF17

FGF9

PAN3

WASF3

FLT1

CDK8

RPL21

HSPH1

FRY

N4BP2L1

KATNAL1

PDS5B

DCLK1

TRPC4

NBEA

FOXO1

KBTBD6

TPT1

COG3

TSC22D1

RB1

ZC3H13

FNDC3A

LCP1

LRCH1

INTS6

PCDH17

SUGT1

PCDH9

KLF12

DACH1

RBM26

MYCBP2

SLITRK1

SLAIN1

SPRY2

TM9SF2

DNAJC3

IPO5

MBNL2

DOCK9

EFNB2

TPP2

ARGLU1

TUBGCP3

ATP11A

ARHGEF7

COL4A1

TNFSF13B

MYO16

LAMP1

TFDP1

CHAMP1

CDC16

CUL4A 
CHD8

ZNF219

SUPT16H

HNRNPC

MMP14

PRMT5

ACIN1

RNF31

CPNE6

JPH4

NFATC4

SCFD1

HECTD1

NOVA1

AKAPG

NPAS3

SRP54

ARHGAP5

PSMA6

SNX6

BAZ1A

NFKBIA

RALGAPA1

MDGA2

FBXO33

PRPF39

MGAT2

SOS2

MAP4K5

ATL1

NEMF

TRIM9

BMP4

DDHD1

STYX

PSMC6

FERMT2

SAMD4A

NAA30

$\mathrm{GCH} 1$

PELI2

EXOC5

ARID4A

RTN1

PSMA3

DAAM1

TRMT5

PRKCH

PPP2R5E

SIX1

PPM1A

HIF1A

GPHN

MAX

EIF2S1

FUT8

ZBTB1

SPTB

ACTN1

DCAF5

RBM25

PSEN1

SIPA1L1

PCNX

MAP3K9

ZFYVE1

ELMSAN1 
FCF1

YLPM1

DLST

TMEM63C

TGFB3

IRF2BPL

GTF2A1

NRXN3

AHSA1

SNW1

RPS6KA5

FOXN3

TTC7B

PSMC1

ZC3H14

ITPK1

SMEK1

FBLN5

UNC79

DICER1

BCL11B

PAPOLA

WARS

YY1

EVL

CCNK

EML1

DYNC1H1

TECPR2

WDR20

TRAF3

CDC42BPB

RCOR1

PPP2R5C

PPP1R13B

EIF5

INF2

JAG2

MTA1

AKT1

PACS2

MAGEL2

CYFIP1

GABRA5

GABRB3

HERC2

GABRG3

GOLGA8M

UBE3A

TJP1

EMC7

OTUD7A

RYR3

ACTC1

EMC4

AQR

LPCAT4

MEIS2

SLC12A6

THBS1

BAHD1

VPS18

INO80

DLL4

RAD51

MAPKBP1

MGA 
RTF1

TTBK2

MAP1A

CDAN1

TP53BP1

FRMD5

PDIA3

MFAP1

CTDSPL2

MYEF2

SLC30A4

SEMA6D

USP8

AP4E1

COPS2

FBN1

GABPB1

MYO5A

DMXL2

LEO1

RSL24D1

FAM214A

TCF12

CCPG1

RFX7

PYGO1

ADAM10

RNF111

TLN2

RORA

SLTM

HERC1

USP3

CSNK1G1

ZNF609

CTD-2116N17.1

SNX1

DPP8

MAP2K1

RPL4

RAB11A

KIF23

TLE3

PIAS1

ANP32A

PARP6

NEO1

ARIH1

PKM

NPTN

MYO9A

ARID3B

CLK3

PML

CSK

SIN3A

PTPN9

C15orf39

LINGO1

HMG20A

IREB2

RASGRF1

ARNT2

AP3B2

HDGFRP3

CPEB1

ZNF592 
ACAN

NTRK3

ZNF710

FURIN

IQGAP1

CRTC3

CHD2

NR2F2

ASB7

CHSY1

ALDH1A3

SNRPA1

RAB11FIP3

CAPN15

FBXL16

UBE2I

TMEM204

MAPK8IP3

CLCN7

CRAMP1L

PKD1

TSC2

CASKIN1

RNPS1

PDPK1

NAA60

CREBBP

GLIS2

ADCY9

TFAP4

C16orf72

GLYR1

GRIN2A

UBN1

RBFOX1

USP7

GSPT1

ZC3H7A

MKL2

KIAA0430

SMG1

MYH11

XYLT1

RBBP6

TNRC6A

CACNG3

PRKCB

PLK1

ARHGAP17

XPO6

GTF3C1

ATXN2L

IL27

SH2B1

TAOK2

MAZ

CORO1A

TBC1D10B

ZNF629

RNF40

SRCAP

FBRS

FUS

KAT8

SETD1A

STX1B

FBXL19 
VPS35

SIAH1

DNAJA2

ZNF423

PAPD5

ITFG1

NKD1

BRD7

RBL2

CHD9

CYLD

TOX3

SALL1

GNAO1

NUDT21

RSPRY1

FAM192A

CX3CL1

ZNF319

$\mathrm{CDH} 11$

CNOT1

$\mathrm{CDH} 8$

CSNK2A2

CBFB

ATP6V0D1

CTCF

RANBP10

EDC4

NFATC3

WWP2

VPS4A

TERF2

NFAT5

AP1G1

ST3GAL2

SF3B3

DDX19A

GLG1

ZFHX3

BCAR1

CMIP

PLCG2

KLHL36

USP10

FBXO31

$\mathrm{JPH} 3$

ZCCHC14

FOXF1

IRF8

BANP

ZC3H18

ANKRD11

RPL13

TUBB3

YWHAE

PITPNA

ABR

SMG6

MNT

PRPF8

METTL16

PAFAH1B1

CLUH

RAP1GAP2

ZZEF1

ANKFY1

PELP1 
C17orf85

CAMTA2

MINK1

PITPNM3

RABEP1

DERL2

PHF23

DLG4

NEURL4

YBX2

NLGN2

CTDNEP1

EIF4A1

TNFSF12

SENP3

POLR2A

FXR2

ZBTB4

TNFSF12-TNFSF13

TP53

KDM6B

CHD3

MYH10

RPL26

NDEL1

MAP2K4

MYOCD

ARHGAP44

FLCN

RAI1

NCOR1

COPS3

LGALS9C

SREBF1

LLGL1

GID4

AKAP10

WSB1

USP22

NLK

SUPT6H

FOXN1

TAOK1

PHF12

GIT1

NUFIP2

MYO18A

NF1

CRLF3

ATAD5

SSH2

ANKRD13B

LRRC37B

PSMD11

RHOT1

C17orf75

SUZ12

RAB11FIP4

ASIC2

AP2B1

GGNBP2

ACACA

MLLT6

HNF1B

SRCIN1

PIP4K2B

SYNRG 


\begin{tabular}{|c|}
\hline SOCS7 \\
\hline RPL23 \\
\hline CACNB1 \\
\hline RPL19 \\
\hline FBXL20 \\
\hline CDK12 \\
\hline ERBB2 \\
\hline MED1 \\
\hline CASC3 \\
\hline MSL1 \\
\hline NR1D1 \\
\hline RARA \\
\hline IKZF3 \\
\hline PSMD3 \\
\hline SMARCE1 \\
\hline TOP2A \\
\hline KRT31 \\
\hline ACLY \\
\hline KLHL10 \\
\hline DNAJC7 \\
\hline TUBG1 \\
\hline ATP6V0A1 \\
\hline STAT5B \\
\hline STAT3 \\
\hline $\mathrm{KCNH} 4$ \\
\hline STAT5A \\
\hline BECN1 \\
\hline PSME3 \\
\hline DHX8 \\
\hline UBTF \\
\hline SLC4A1 \\
\hline RUNDC3A \\
\hline LSM12 \\
\hline C17orf104 \\
\hline HDAC5 \\
\hline GPATCH8 \\
\hline ATXN7L3 \\
\hline HEXIM1 \\
\hline NMT1 \\
\hline GJC1 \\
\hline EFTUD2 \\
\hline FMNL1 \\
\hline NPEPPS \\
\hline KANSL1 \\
\hline KPNB1 \\
\hline WNT3 \\
\hline NFE2L1 \\
\hline SP2 \\
\hline TBX21 \\
\hline CBX1 \\
\hline UBE2Z \\
\hline IGF2BP1 \\
\hline ZNF652 \\
\hline PPP1R9B \\
\hline KAT7 \\
\hline COL1A1 \\
\hline SPOP \\
\hline CA10 \\
\hline SPAG9 \\
\hline LUC7L3 \\
\hline CACNA1G \\
\hline MBTD1 \\
\hline MSI2 \\
\hline SRSF1 \\
\hline VEZF1 \\
\hline MTMR4 \\
\hline USP32 \\
\hline
\end{tabular}


CLTC

VMP1

YPEL2

PPM1E

APPBP2

DHX40

RPS6KB1

BCAS3

MRC2

MED13

TANC2

INTS2

TLK2

TBX2

PSMC5

DDX42

ERN1

CD79B

SMARCD2

MAP3K3

PSMD12

BPTF

PITPNC1

DDX5

SMURF2

HELZ

PRKCA

SOX9

PRKAR1A

MAP2K6

NUP85

CASKIN2

UNK

SLC25A19

UBE2O

TNRC6C

CYTH1

CBX8

CBX2

RPTOR

EIF4A3

NPLOC4

RP11-1055B8.7

FASN

GPS1

P4HB

CSNK1D

FOXK2

COLEC12

USP14

SMCHD1

TYMS

DLGAP1

PTPRM

ANKRD12

PPP4R1

GNAL

PTPN2

ESCO1

ROCK1

C18orf8

KCTD1

SS18

ZNF521

ASXL3

NOL4

DSG1 


\begin{tabular}{|c|}
\hline TRAPPC8 \\
\hline DTNA \\
\hline B4GALT6 \\
\hline MAPRE2 \\
\hline SETBP1 \\
\hline ZNF24 \\
\hline CELF4 \\
\hline FHOD3 \\
\hline ATP5A1 \\
\hline RNF165 \\
\hline PIAS2 \\
\hline C18orf25 \\
\hline CXXC1 \\
\hline ZBTB7C \\
\hline SMAD7 \\
\hline CTIF \\
\hline SMAD2 \\
\hline MBD1 \\
\hline TXNL1 \\
\hline WDR7 \\
\hline MBD2 \\
\hline ONECUT2 \\
\hline DCC \\
\hline MEX3C \\
\hline TCF4 \\
\hline SMAD4 \\
\hline ST8SIA3 \\
\hline NEDD4L \\
\hline KIAA1468 \\
\hline MALT1 \\
\hline ZNF532 \\
\hline $\mathrm{CDH} 2 \mathrm{O}$ \\
\hline PHLPP1 \\
\hline ZNF407 \\
\hline NETO1 \\
\hline TSHZ1 \\
\hline NFATC1 \\
\hline CTDP1 \\
\hline ZNF236 \\
\hline MIER2 \\
\hline PTBP1 \\
\hline WDR18 \\
\hline STK11 \\
\hline MBD3 \\
\hline DAZAP1 \\
\hline APC2 \\
\hline DOT1L \\
\hline CSNK1G2 \\
\hline BTBD2 \\
\hline LMNB2 \\
\hline FZR1 \\
\hline GNA11 \\
\hline CELF5 \\
\hline CACTIN \\
\hline EEF2 \\
\hline PIAS4 \\
\hline DPP9 \\
\hline KDM4B \\
\hline SAFB2 \\
\hline SAFB \\
\hline CHAF1A \\
\hline RANBP3 \\
\hline MLLT1 \\
\hline LONP1 \\
\hline RFX2 \\
\hline VAV1 \\
\hline C3 \\
\hline
\end{tabular}


KHSRP

ARHGEF18

CAMSAP3

XAB2

ELAVL1

EVI5L

MAP2K7

HNRNPM

ADAMTS10

OLFM2

EIF3G

PDE4A

CDC37

DNMT1

C19orf66

RAVER1

CARM1

SMARCA4

DNM2

ILF3

KANK2

DKFZP761J1410

MAST1

TNPO2

CALR

CACNA1A

NFIX

NACC1

RFX1

LPHN1

PRKACA

DCAF15

SLC1A6

SYDE1

BRD4

WIZ

AKAP8

EPS15L1

RAB8A

SIN3B

MYO9B

CHERP

MED26

RPL18A

$\mathrm{FCHO} 1$

JAK3

UNC13A

ANO8

ELL

FKBP8

PIK3R2

MAST3

SSBP4

KCNN1

LRRC25

CRTC1

UPF1

SUGP2

SUGP1

ATP13A1

MAU2

GATAD2A

ZNF536

UBA2

HPN

USF2

KMT2B 
CLIP3

SIPA1L3

DPF1

HNRNPL

ACTN4

MAP4K1

SAMD4B

SUPT5H

DYRK1B

AKT2

FBL

HNRNPUL1

LTBP4

RAB4B

RPS19

CD79A

GRIK5

MEGF8

GSK3A

$\mathrm{CIC}$

ZNF574

POU2F2

ARHGEF1

ATP1A3

CADM4

TOMM40

ZNF296

RELB

EXOC3L2

CLASRP

CLPTM1

MARK4

SYMPK

IRF2BP1

PPP5C

PRKD2

NPAS1

SAE1

ZC3H4

ARHGAP35

STRN4

NAPA

AP2S1

GLTSCR1

GRIN2D

RASIP1

CA11

RUVBL2

SNRNP70

PPFIA3

FLT3LG

SLC17A7

RPS11

PRR12

AP2A1

PRMT1

TSKS

SCAF1

MYH14

SHANK1

PRKCG

TTYH1

PRPF31

CNOT3

LENG8

BRSK1

PPP6R1 
U2AF2

ZNF628

RPS5

TRIM28

CHMP2A

CSNK2A1

TBC1D20

STK35

PTPRA

NOP56

ATRN

CENPB

PLCB1

BMP2

PCNA

SNAP25

FLRT3

TASP1

PAK7

JAG1

ZNF133

PCSK2

SLC24A3

NAPB

TPX2

C20orf112

TM9SF4

MAPRE1

XKR7

POFUT1

DNMT3B

E2F1

CBFA2T2

ITCH

AHCY

GDF5

TRPC4AP

GGT7

NCOA6

DLGAP4

TGIF2-C20orf24

SOGA1

RBM39

NDRG3

PHF20

PPP1R16B

RALGAPB

SRC

PTPRT

MYBL2

CHD6

TOP1

SRSF6

YWHAB

PABPC1L

RIMS4

HNF4A

NCOA5

SLC12A5

PCIF1

ZNFX1

CSE1L

KCNB1

ZMYND8

STAU1

ARFGEF2

NCOA3 
PREX1

B4GALT5

ATP9A

ADNP

NFATC2

SALL4

ZNF217

TFAP2C

CSTF1

BMP7

GNAS

RAE1

TAF4

SYCP2

$\mathrm{CDH} 4$

PSMA7

PHACTR3

LSM14B

TCFL5

MRGBP

LAMA5

GID8

YTHDF1

DIDO1

ADRM1

KCNQ2

EEF1A2

GMEB2

MYT1

ZNF512B

NRIP1

USP25

NCAM2

CCT8

GABPA

TIAM1

HUNK

SCAF4

SON

PAXBP1

ITSN1

MORC3

ETS2

BRWD1

DYRK1A

ERG

BACE2

DSCAM

ZBTB21

SIK1

U2AF1

TRAPPC10

PKNOX1

AGPAT3

PDXK

COL6A1

PCBP3

MICAL3

TBX1

HIRA

sep-05

UFD1L

DGCR8

ZDHHC8

MED15

HIC2

MAPK1 
BCR

SMARCB1

SPECC1L

SEZ6L

SGSM1

PITPNB

NIPSNAP1

AP1B1

NF2

ZNRF3

EWSR1

TBC1D10A

MTMR3

SF3A1

EIF4ENIF1

MORC2

DRG1

PRR14L

DEPDC5

PATZ1

LARGE

MYH9

CACNG2

EIF3D

RBFOX2

IL2RB

GGA1

CSNK1E

EIF3L

SOX10

GTPBP1

RPL3

PDGFB

CACNA1I

RBX1

TNRC6B

EP300

ZC3H7B

SREBF2

XRCC6

TCF20

SULT4A1

SCUBE1

PRR5

SMC1B

PHF21B

CELSR1

ZBED4

GRAMD4

BRD1

TBC1D22A

PLXNB2

PIM3

TRABD

SHANK3

MAPK8IP2

KAL1

WWC3

NLGN4X

TBL1X

STS

CLCN4

PRPS2

HCCS

GLRA2

OFD1

ARHGAP6 
GEMIN8

FRMPD4

MSL3

TLR8

PIGA

RBBP7

FANCB

TXLNG

ZRSR2

ACE2

MOSPD2

CDKL5

REPS2

PDHA1

GPR64

SCML2

NHS

PPEF1

CNKSR2

SCML1

SH3KBP1

RPS6KA3

PHKA2

POLA1

PTCHD1

EIF2S3

ZFX

SMS

MBTPS2

PHEX

PCYT1B

KLHL15

TAB3

DMD

IL1RAPL1

GK

TM4SF2

RPGR

BCOR

OTC

CYBB

CXorf22

CASK

MED14

MAOB

DDX3X

MAOA

USP9X

KDM6A

SLC9A7

EFHC2

USP11

UBA1

PHF16

CDK16

RBM10

CFP

FTSJ1

PORCN

WAS

WDR13

SUV39H1

SLC38A5

GPKOW

WDR45

GRIPAP1

TFE3 
OTUD5

HDAC6

AF196779.12

CCDC22

FOXP3

GSPT2

AKAP4

SHROOM4

CLCN5

MAGED1

IQSEC2

RIBC1

HUWE1

KDM5C

SMC1A

WNK3

PHF8

FGD1

MAGED2

USP51

FAM120C

ALAS2

GNL3L

MSN

ARHGEF9

ZC3H12B

AMER1

IGBP1

EDA

OPHN1

AR

CXorf65

ZMYM3

TAF1

TEX11

MED12

KIF4A

SLC7A3

IL2RG

NONO

OGT

ACRC

DLG3

HDAC8

RLIM

SLC16A2

KIAA2022

ABCB7

ERCC6L

ATRX

TBX22

ATP7A

PGK1

ZNF711

PCDH11X

BRWD3

CHM

DIAPH2

PCDH19

CENPI

GLA

SRPX2

BTK

PLP1

FAM199X

MORC4

IL1RAPL2 
FRMPD3

AMMECR1

MID2

ACSL4

COL4A6

ATG4A

COL4A5

RGAG1

AMOT

ALG13

LRCH2

PAK3

CAPN6

SLC6A14

TRPC5

PLS3

NKAP

UPF3B

CXorf56

WDR44

sep-06

DOCK11

NKRF

IL13RA1

LAMP2

CUL4B

TENM1

XIAP

THOC2

OCRL

SMARCA1

STAG2

GRIA3

UTP14A

BCORL1

AlFM1

ARHGAP36

SLC25A14

ZNF280C

FRMD7

IGSF1

PHF6

DDX26B

GPC3

HPRT1

GPC4

FHL1

FGF13

SLC9A6

MCF2

HTATSF1

ARHGEF6

F9

RBMX

ATP11C

IDS

MTM1

AFF2

MTMR1

NSDHL

BGN

HAUS7

SLC6A8

ATP2B3

IDH3G

ABCD1

IRAK1 
PDZD4

RENBP

HCFC1

OPN1LW

ARHGAP4

L1CAM

FLNA

G6PD

RPL10

TKTL1

GDI1

TAZ

FAM50A

PLXNA3

MPP1

GAB3

DKC1 